\title{
Whole-transcriptome Analysis of Fully Viable Energy Efficient Glycolytic-null Cancer Cells Established by Double Genetic Knockout of Lactate Dehydrogenase A/B or Glucose-6-Phosphate Isomerase
}

\author{
ELIZABETH MAZZIO ${ }^{1}$, RAMESH BADISA ${ }^{1}$, NZINGA MACK ${ }^{1}$, SHAMIR CASSIM ${ }^{2}$, \\ MASA ZDRALEVIC ${ }^{3 \#}$, JACQUES POUYSSEGUR ${ }^{2,3}$ and KARAM F.A. SOLIMAN ${ }^{1}$ \\ ${ }^{1}$ College of Pharmacy \& Pharmaceutical Sciences, Florida A\&M University, Tallahassee, FL, U.S.A.; \\ ${ }^{2}$ Department of Medical Biology, Centre Scientifique de Monaco, Monaco, Monaco; \\ ${ }^{3}$ University Côte d'Azur, IRCAN, CNRS, Centre A. Lacassagne, Nice, France
}

\begin{abstract}
Background/Aim: Nearly all mammalian tumors of diverse tissues are believed to be dependent on fermentative glycolysis, marked by elevated production of lactic acid and expression of glycolytic enzymes, most notably lactic acid dehydrogenase (LDH). Therefore, there has been significant interest in developing chemotherapy drugs that selectively target various isoforms of the LDH enzyme. However, considerable questions remain as to the consequences of biological ablation of $\mathrm{LDH}$ or upstream targeting of the glycolytic pathway. Materials and Methods: In this study, we explore the biochemical and whole transcriptomic effects of CRISPR-Cas9 gene knockout (KO) of lactate dehydrogenases $A$ and $B[L D H A / B$ double $K O$ (DKO)] and glucose-6-phosphate isomerase (GPI KO) in the human colon cancer cell line LS174T, using Affymetrix 2.1 ST arrays. Results: The metabolic biochemical profiles corroborate that relative to wild type (WT), LDHA/B DKO produced no lactic acid, (GPI KO) produced minimal lactic acid and both KOs displayed higher mitochondrial respiration, and minimal use of glucose with no loss of cell
\end{abstract}

This article is freely accessible online.

\#Present address: Faculty of Medicine, University of Montenegro, Kruševac bb, 81000 Podgorica, Montenegro.

Correspondence to: Karam F.A. Soliman, Room G 134 H New Pharmacy Building, 1415 ML King Blvd, Tallahassee, FL 32307, U.S.A. Tel: +1 8505993306, e-mail: karam.soliman@famu.edu; Jacques Pouyssegur, Centre Scientifique de Monaco, CSM, 98000 Monaco, Monaco. E-mail: Jacques.pouyssegur@unice.fr

Key Words: Cancer, metabolism, LDHA, LDHB, GPI, Warburg effect, genes. viability. These findings show a high biochemical energy efficiency as measured by ATP in glycolysis-null cells. Next, transcriptomic analysis conducted on 48,226 mRNA transcripts reflect 273 differentially expressed genes (DEGS) in the GPI KO clone set, 193 DEGS in the LDHA/B DKO clone set with 47 DEGs common to both KO clones. Glycolytic-null cells reflect up-regulation in gene transcripts typically associated with nutrient deprivation / fasting and possible use of fats for energy: thioredoxin interacting protein (TXNIP), mitochondrial 3-hydroxy-3-methylglutaryl-CoA synthase 2 (HMGCS2), PPAR $\gamma$ coactivator $1 \alpha(P G C-1 \alpha)$, and acetyl-CoA acyltransferase 2 (ACAA2). Other changes in non-ergometric transcripts in both KOs show losses in "stemness", WNT signaling pathway, chemo/radiation resistance, retinoic acid synthesis, drug detoxification, androgen/estrogen activation, and extracellular matrix reprogramming genes. Conclusion: These findings demonstrate that: 1) The "Warburg effect" is dispensable, 2) loss of the LDHAB gene is not only inconsequential to viability but fosters greater mitochondrial energy, and 3) drugs that target LDHA/B are likely to be ineffective without a plausible combination second drug target.

Common features across diverse mammalian tumors include the rapid glycolytic activity, greater expression of lactate dehydrogenases (LDH) and subsequent over-production of lactic acid, occurring regardless of the presence of oxygen (Warburg effect) $(1,2)$. This unique trait of cancer has been studied for decades, where researchers have geared off original focus from its role in metabolism to its role in acidification of the tumor microenvironment (TME), a formidable driving force for tumor growth, metastasis, aggressiveness, chemo/radiation resistance and loss of immune surveillance (3-8). This loss of immune surveillance 
involves a concurrent loss of host $\mathrm{T}$ and NK cell functions combined with tumor sequestration of immune leukocyte subpopulations, infiltrated in solid acidic tumors, promoting tumor survival $(7,9)$. TME acidification directly correlates to tumor progression, which has been exploited pharmacologically for the development of drug delivery systems such as low $\mathrm{pH}$ insertion peptides pHLIP $(10,11)$, or delivery diagnostic tools (12), while attempts at reducing acidity genetically or by pharmacological means include drugs that target LDHA and lactate $/ \mathrm{H}^{+}$symporters (13).

Previously, our laboratory conducted a high throughput screening of over a thousand natural herbs and phytochemicals for inhibition of LDHA (14). LDHA inhibitors, once identified, were then evaluated for their effects on metabolic parameters in MDA-MB-231 breast cancer cells, showing no cell death or reduced production of lactic acid, with the only observable effects being the reduction in proliferative rate (15). Subsequently, we silenced the LDHA gene in MDA-MB-231 cells, where we again reconfirm no adverse consequential effects on viability, lactic acid, energy, or any other observable malignant attribute (16). Subsequent studies led us to investigate all LDH isoforms in this particular cell line - revealing a high dominance of co-existent LDHA and B. We suggested that a double knockdown would be required to reduce both $L D H A / B$ gene expressions to accurately identify the consequences to the loss of this gene. At the same time, Pouyssegur's research group created dual (LDHA/B) knock out clones using CRISPR-Cas9 genome editing, where they reported that this double genetic disruption completely eliminated lactic acid production in human colon adenocarcinoma (LS174T) and mouse melanoma (B16) cells, having no effect on viability, yet coinciding with greater mitochondrial OXPHOS capacity $(17,18)$. They also established a clone with complete genetic ablation of glucose-6-phosphate isomerase (GPI), which, similarly to the $L D H A / B$ DKO, showed reduced lactic acid production, greater reliance on OXPHOS and suppressed growth in hypoxia (18-20).

Given the unusual survival of tumor cells in response to the severe compromise of glycolysis, in this work we used Affymetrix whole-transcriptome microarrays combined with metabolic biochemical evaluation to explore survival mechanisms.

\section{Materials and Methods}

Materials. Ninety-six well plates, pipette tips, Dulbecco's modified Eagle's medium (DMEM), fetal bovine serum (FBS), penicillin/streptomycin, general reagents and supplies were all purchased from Sigma-Aldrich Co. (St. Louis, MO, USA) and VWR International (Radnor, PA, USA). All microarray equipment, reagents, and materials were purchased from Affymetrix/ Thermo Fisher (Waltham, MA, USA).
Cell culture. Dukes' type B, colorectal adenocarcinoma LS174T (ATCC ${ }^{\circledR} \mathrm{CL}-188^{\mathrm{TM}}$ ) WT versus GPI KO and $L D H A / B$ double $\mathrm{KO}$ (DKO) were established and provided by Dr. Pouyssegur's lab $(17,18)$. The cells were cultured in $75 \mathrm{~cm}^{2}$ flasks containing high glucose DMEM supplemented with 6\% FBS and 1\% 10,000 U/ml penicillin $\mathrm{G}$ sodium $/ 10,000 \mu \mathrm{g} / \mathrm{ml}$ streptomycin sulfate. Cells were grown at $37^{\circ} \mathrm{C}$ in $95 \%$ atmosphere $5 \% \mathrm{CO}_{2}$ and sub-cultured every three to five days. The WT control grew in multi-cellular aggregates, which required trypsin detachment [Trypsin-EDTA $(0.25 \%)$, phenol red] on a regular basis through pressurized distribution using a $5 \mathrm{ml}$ serological pipette, deactivation with FBS growth media, and monolayer re-seeding. Both $\mathrm{KO}$ clones grew in relatively well-distributed monolayers. High glucose DMEM $(4,500 \mathrm{mg} / \mathrm{l})$ was used for all studies, except for monitoring glucose consumption, which required low glucose DMEM $(1000 \mathrm{mg} / \mathrm{l})$ to unmask differential rates between the three clones.

Cell viability. Cells were equally plated in 96 well plates at $5 \times 10^{5}$ cells/ml in high glucose media. The resazurin (alamar blue) indicator dye was used to measure viability (21). Briefly, a working solution of resazurin $(0.5 \mathrm{mg} / \mathrm{ml})$ was prepared in sterile PBS and filter sterilized through a 0.2 -micron filter. The dye solution was added to the samples [15\% (v/v) equivalent], and samples were then returned to the incubator for 6-12 h. Reduction of the dye by viable cells reduced the oxidized resazurin, yielding a bright red fluorescent intermediate resorufin quantified using a Synergy HTX multi-mode reader (Bio-Tek, Winooski, VT, USA) with the following settings: $530 \mathrm{~nm}$ (excitation) $/ 590 \mathrm{~nm}$ (emission) filters.

Lactic acid. Determination of lactic acid produced and released in the media by the three clones was done from cells plated at a density of $5 \times 10^{5}$ cells $/ \mathrm{ml}$ by dual detection. Lactic acid was quantified by HPLC (Shimadzu), equipped with an SPD-20A UV detector (set at $210 \mathrm{~nm}$ ), a workstation containing EZSTART version 7.4 software and an SS420X instrument interface docked to a Waters Autosampler Model 717 Plus (Shimadzu Scientific Instruments, Columbia, MD, USA; Waters, Milford, MA, USA). The flow rate was isocratic at $0.6 \mathrm{ml} / \mathrm{min}$. The mobile phase contained $5 \mathrm{mM}$ sulfuric acid, and the column used was an Aminex HPX-87H $300 \times 7.8$ mm; $9 \mu \mathrm{m}$ particle size (Bio-Rad, Hercules, CA, USA). Lactic acid was also determined using a colorimetric enzymatic assay using lactate reagent protocol as provided by the manufacturer (Trinity Biotech, Jamestown, NY, USA). The reagent was added to the samples and incubated for 8 minutes. Lactate was quantified at $490 \mathrm{~nm}$ using a Synergy HTX multi-mode reader (Bio-Tek). Experimental media for this assay was high glucose DMEM, containing $1 \%$ FBS, $4 \mathrm{mM}$ L-glutamine, and penicillin/streptomycin $(100 \mathrm{U} / 0.1 \mathrm{mg} / \mathrm{ml})$ (minus phenol red). Lower FBS was required to carry out the colorimetric assay, as it interferes with the lactate reagent.

Glucose consumption. Briefly, glucose was quantified by an enzymatic assay using glucose oxidase $(20 \mathrm{U} / \mathrm{ml})$ and a chromogenic solution, then quantified at $490 \mathrm{~nm}$ on the Synergy/HTX multi-mode reader (Bio-Tek). Experimental media for this assay was DMEM (glucose $1000 \mathrm{mg} / \mathrm{l}$ ) containing $1 \%$ FBS, 4 $\mathrm{mM}$ L-glutamine and penicillin/streptomycin $(100 \mathrm{U} / 0.1 \mathrm{mg} / \mathrm{ml})$ (minus phenol red). 
Somatic cell ATP. Adenosine triphosphate (ATP) was assessed by using the Adenosine 5'-triphosphate (ATP) bioluminescent somatic cell assay kit (Catalog Number FLASC) purchased from Sigma Aldrich (St. Louis, MO, USA) and carried out according to the manufacturer's instructions. Briefly, cells were lysed with ATP releasing reagent, transferred to a flat white bottom 96 well plate and quantified on a Synergy HTX multi-mode reader (Bio-Tek) using luminescence settings.

Oxygen consumption. The oxygraph respirometer-Clark electrode (Hansatech Instruments Ltd., Norfolk, England) was used to evaluate cellular/mitochondrial respiration indirectly by evaluating dissolved $\mathrm{O}_{2}$ in the media. The electrode was calibrated with both air saturated deionized water and deionized water bubbled with nitrogen for 20 minutes, with additional sodium dithionite to stabilize zero oxygen line calibration. Briefly, the cell supernatants or control media blanks were loaded into the cell chamber jacket at $25^{\circ} \mathrm{C}$. After rate equilibration, a reading was taken, and data were plotted as $\mu \mathrm{M} \mathrm{O}_{2}$ consumed/ $24 \mathrm{~h}$.

Microarray WT 2.1 human datasets. All cells were washed three times in ice-cold HBSS, rapidly frozen and stored at $-80^{\circ} \mathrm{C}$. Total RNA was isolated and purified using the Trizol/chloroform method. The quality was assessed, and concentration was equalized to $82 \mathrm{ng} / \mu \mathrm{l}$ in nucleasefree water. Whole transcriptome analysis was conducted according to the GeneChipTM WT PLUS Reagent Manual for Whole Transcript (WT) Expression Arrays. Briefly, RNA was reverse transcribed to firststrand /second-strand cRNA, cRNA was purified and assessed for yield, prior to the second cycle single-stranded cDNA synthesis, hydrolysis of RNA, and purification of the second cycle single-stranded cDNA. Subsequently, cDNA was assessed for yield, then fragmented, labeled, and hybridized onto the arrays prior to being subjected to fluidics and imaging using the Gene Atlas (Affymetrix, ThermoFisher Scientific). The array data quality control and initial processing from CEL to CHP files were conducted using an expression console, prior to data evaluation using the Affymetrix transcriptome analysis console and String Database (String Consortium 2020) (22, 23). The dataset has been deposited to NIH Gene Expression Omnibus located at: https://www.ncbi.nlm.nih.gov/geo/query/acc.cgi?acc=GSE149289.

Data analysis. Statistical analysis was performed using GraphPad Prism (version 3.0; Graph Pad Software Inc., San Diego, CA, USA) with the significance of difference between the groups assessed using a one-way ANOVA and then followed by Tukey post hoc analysis.

\section{Results}

Metabolic profiling. The metabolic parameters in WT and GPI KO LS174T as well as $L D H A / B$ DKO colon adenocarcinoma cells were acquired at $24 \mathrm{~h}$ for viability (Figure 1A), cell respiration (Figure 1B), glucose consumption (Figure 1C), lactic acid production/release (Figure 1D) and somatic ATP generation (Figure 1E). The findings clearly suggest that both subset KO LS174T viable clones are capable of maintaining energy to thrive, with the primary difference being that the GPI KO and $L D H A / B$ DKO clones display impaired glycolytic ability, as evidenced by the severe loss of lactic acid production, occurring to a greater extent in the latter subset $(L D H A / B$ DKO). These data also show greater ATP yield in both knockouts compared to the WT, with no detectable levels of glucose being consumed within the first $48 \mathrm{~h}$. Experimental protocols to sufficiently quantify glucose consumption rates required low glucose media $(1000 \mathrm{mg} / \mathrm{l})$, with data obtained when controls consumed most of the glucose in 96 well plates with an endpoint at $48 \mathrm{~h}$ (Figure 1C). This experimental design clearly shows a difference in the WT controls which utilize glucose at a fairly rapid rate, tantamount to the production of lactic acid. In summary, baseline studies confirm that fermentative glycolysis is not required for cells to maintain energy systems, cells can overcome a complete loss of $L D H A / B$ where there appears to be a significant switch to an efficient ATP producing system, non-reliant on glucose. In order to investigate how $\mathrm{KO}$ clones overcome a nearcomplete loss of glycolysis, whole transcriptomic microarrays were then conducted.

Microarray. The overall bio statistical summary data for the microarrays were quantified according to the following analysis set parameters: Fold Change: $>2$ or $<-2$, $p$ Value $<0.05$, and filtered for only characterized genes. The summary analysis report shows 48,226 genes evaluated, 273 deferentially expressed genes (DEGS) were found in the GPI KO clone set, 193 DEGS in the $L D H A / B$ DKO clone set with 47 DEGs consistent to both KO clones (Figure 2). These changes correspond to transcriptomic shifts being less than $0.5 \%$ of the transcriptome affected in both glycolysisnull KOs (Figure 3A, B). Detailed data on the DEGs are presented for both independent clones (Tables I and II) and DEGs consistent to both clones (Table III).

DEG tables. Table I reflects the most significant shift in DEGs for the $L D H A / B$ DKO clone set versus WT controls, where the highest down-regulated genes were: mucin 6 (MUC6), dickkopf (WNT signaling pathway inhibitor) 4 (DKK4), alanyl (membrane) aminopeptidase (ANPEP), EPS8-like 3 (EPS8L3) and solute carrier family 44 member 4 (SLC44A4), while the highest up-regulated genes were among others: cilia and flagella associated protein 126 (CFAP126), 2-5-oligoadenylate synthetase 2 (OAS2), neurotensin (NTS) and serine peptidase inhibitor, Kazal type 1 (SPINK1).

Table II reflects the most significant shift in DEGs for the GPI KO clone set versus WT controls, where down-regulated genes show the highest losses for: dickkopf WNT signaling pathway inhibitor $4(D K K 4)$, gap junction protein alpha 1 (GJAl), versican $(V C A N)$, biglycan $(B G N)$, and the top up-regulated genes were: S100 calcium-binding protein A7 (S100A7), v-kit Hardy-Zuckerman 4 feline sarcoma viral oncogene homolog (KIT), 5-hydroxytryptamine (serotonin) receptor $1 \mathrm{D}, \mathrm{G}$ proteincoupled (HTRID) and 3-hydroxy-3-methylglutaryl-CoA synthase 2, which is mitochondrial (HMGCS2). 

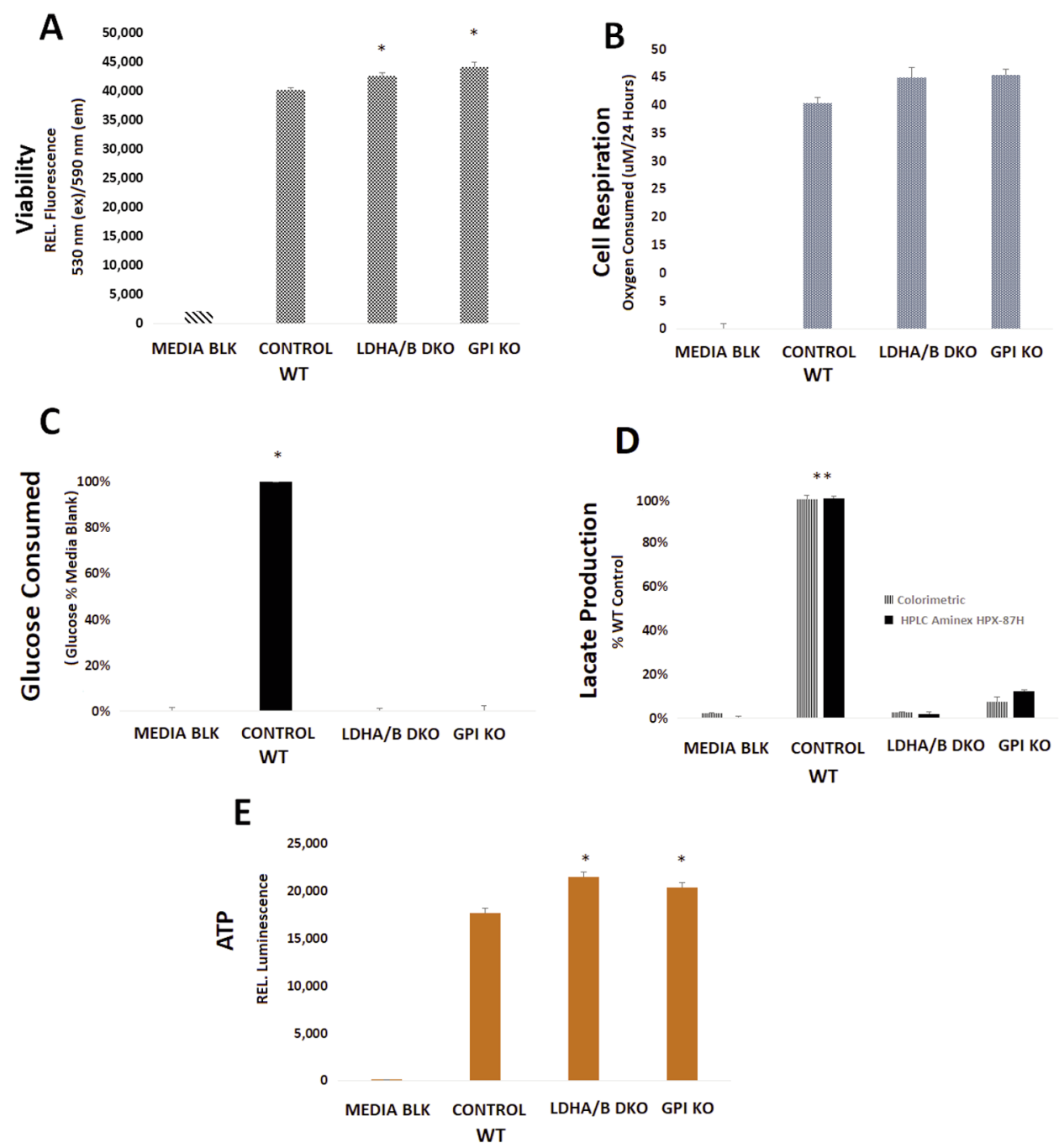

Figure 1. Metabolic parameters in the control WT (LS174T) cells, relative to LDHA/B double knockout (DKO) and GPI KO including: (A) basic viability [relative fluorescent intensity defined as $530($ ex)/590 (em)], (B) oxygen respiration (O2 consumed $\mu$ M/ 24 h), (C) glucose consumption (media glucose concentrations as a \% of WT controls with no defect in glycolysis), (D) lactic acid production (lactic acid produced as a \% of WT controls with no defect in glycolysis) and (E) somatic ATP concentrations (relative luminescent values). The data was analyzed by a one way ANOVA, followed by a Tukey post hock test, where significant differences are represented by * $p$-Value $<0.05$ and significant differences established for the following: (A-B) WT versus KO clones, $(C)$ glucose media versus consumption in the 3 clones, $(D)$ lactic acid production in 3 clones versus media blank and (E) WT versus KO clones.

Table III shows only overlapping unidirectional DEGs for both glycolytic KO clones versus WT where down-regulated genes show the most significant losses for: dickkopf WNT signaling pathway inhibitor $4(D K K 4)$, biglycan $(B G N)$, desmocollin 3 (DSC3), crystallin beta B1(CRYBB1), aldoketo reductase family 1 , member $\mathrm{C} 2(A K R I C)$, aldo-keto reductase family 1 , member B10 (aldose reductase) (AKR1B10), and calbindin 2 (CALB2). The top up-regulated genes included among others: serine peptidase inhibitor (SPINK1), Kazal type 1, FXYD domain-containing ion transport regulator 4 (FXYD4), 3-hydroxy-3-methylglutaryl-
CoA synthase 2 (mitochondrial) (HMGCS2), peroxisome proliferator-activated receptor gamma and coactivator 1 alpha (PPARGC1A).

Transcriptome analysis console (TAC) pathway analysis. The totality of the microarray data show that many of the DEGs in clone subsets did not fall on known Wiki metabolic pathways. TAC pathway analysis for glycolysis and TCA cycle genes is shown in Figure 4. In the $L D H A / B$ DKO clones, the data show very few changes accompanying the loss of LDH transcripts, including a reduction in phosphofructokinase (PFKP) with a 


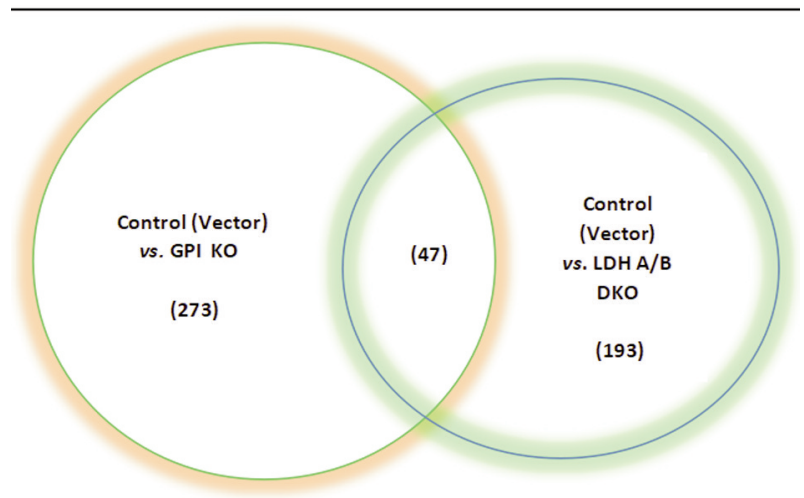

Figure 2. Whole transcriptome summary of deferentially expressed genes (DEG) in both clones (LDHA/B DKO and GPI KO) vs. WT Vector controls in the LS174T colon cancer cell line, and overlapping genes common to both KO clones. Analysis conditions: fold change $>2$ or p-Value $<0.05$. Total number of genes analyzed: 48226. Results: 273 DEGS (GPI KO) and 193 DEGS (LDHA/B DKO) and 47 DEGS common to both KO cell lines.

rise in phosphoenolpyruvate carboxykinase 1 (PCK1), with no modifications to the TCA cycle gene transcripts. The GPI KO clone set shows a compensatory increase in SLC2A1, enolase 2 (ENO2), aldolase, fructose-bisphosphate $C$ (ALDOC) and hexokinase (HK2), with few to no changes in the TCA cyclerelated gene transcripts. Integrated pathway analysis (Figure $5 \mathrm{~A}, \mathrm{~B})$ is also provided, which shows potential changes in pyruvate metabolism, glycolysis and gluconeogenesis, urea cycle, glutamate metabolism, ketone bodies, and fatty acid metabolism. Again, these data show very few additional changes in response to severe glycolytic inhibition: $L D H A / B$ DKO displays reduction in argininosuccinate synthase 1 (ASS1, urea cycle), cystathionine gamma lyase (CTH, cysteine metabolism), aldehyde dehydrogenase 1, family member 1 (ALDH1A1, aldehyde metabolism) and significantly elevated expression of HMGCS2 (Figure 6A), while GPI KO reflects a unique elevation in arginase 2 (ARG2, urea cycle). The prominent overlapping transcriptomic shifts consistent in both clone subsets involve the rise in expression of HMGCS2 in the HMG-CoA cycle Wiki diagram and elevation of nucleartargeted PPARGC1A (Figure 6B).

String_db. String database functional analysis was also conducted on subclone datasets, where the $L D H A / B$ DKO versus WT down-regulated DEGs involved losses in oxidoreductase activities, aldehyde dehydrogenase activities, anion transporter activity, and corresponding increases in transcripts associated with proteins involved with response/defense to viral and other organisms, hexose/fructose metabolism and RIG-I-like receptor signaling (Figure 7A, B). Notably, there was a large integrated response evoked by dual knock down in $L D H A / B$ DKO as
Microarray - Whole Transcriptomic Differentially Expressed Genes
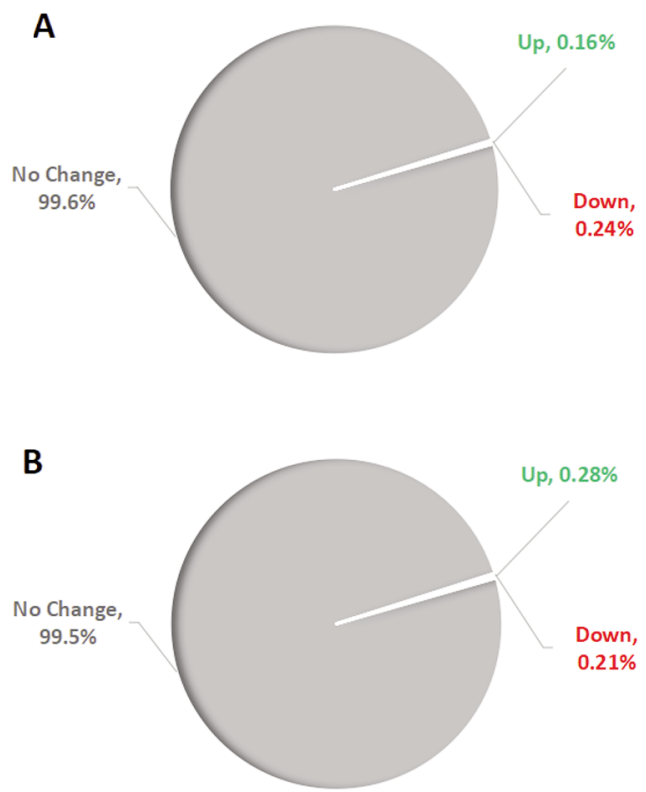

Figure 3. Whole transcriptome summary of DEGs in both clones $(L D H A B D K O)(A)$ and (GPI KO) (B) versus WT vector controls in the $L S 174 T$ colon cancer cell lines, with respect to the entire genome, also reflecting \% of up and down DEGs in both clones. Analysis conditions: fold change $>2$ or p-Value $<0.05$. Total number of genes: 48226 . (A) LDHAB DKO: Up-regulated $78(0.16 \%)$ and Down-regulated 115 (0.24\%), (B) GPI KO: Up-regulated 84 (0.28\%) and Down-regulated $121(0.21 \%)$.

elucidated by this analysis; the reason for this remains unknown. String database functional analysis on the GPI KO versus WT transcriptome was conducted in both directions, where downregulated DEGs involved losses in drug detoxification processes, some specific to doxorubicin/daunorubicin, carboxylic acid metabolic systems, oxidoreductase activity and gluconeogenesis, corresponding to a rise in cell response to hypoxia, HIF1 signaling, oxidation-reduction processes, dioxygenase activity, and hexose metabolic processing (Figure 7C, D).

\section{Discussion}

Energy reprogramming. The data in this study provide evidence to suggest that severe compromise to glycolysis in colon adenocarcinoma LS174T cells can be achieved by selective dual $\mathrm{KO}$ of $L D H A / B$ or single $\mathrm{KO}$ of the glucose-6phosphate isomerase (GPI) gene, and is of little consequence to overall cell survival. Both $\mathrm{KO}$ clones display suppression of lactic acid secretion, either inability or strong reduction in glucose consumption, high production of ATP, greater mitochondrial functions and a slower proliferative growth rate (data not shown), as previously reported $(17,18)$. 
Table I. WT affymetrix human transcriptome: WT versus LDHA/B DKO LS174T colon adenocarcinoma cells.

\begin{tabular}{|c|c|c|c|c|c|c|}
\hline $\begin{array}{l}\text { Control WT } \\
\text { Avg }(\log 2)\end{array}$ & $\begin{array}{l}\text { LDHA/B DKO } \\
\text { Avg }(\log 2)\end{array}$ & $\begin{array}{l}\text { Fold } \\
\text { change }\end{array}$ & $p$-Value & $\begin{array}{c}\text { FDR } \\
p \text {-Value }\end{array}$ & $\begin{array}{l}\text { Gene } \\
\text { symbol }\end{array}$ & Description \\
\hline 8.3 & 4.3 & -15.5 & $2.8 \times 10^{-12}$ & $1.0 \times 10^{-8}$ & MUC6 & Mucin 6, oligomeric mucus/gel-forming \\
\hline 8.4 & 5.0 & -11.0 & $7.5 \times 10^{-8}$ & $3.5 \times 10^{-5}$ & DKK4 & Dickkopf WNT signaling pathway inhibitor 4 \\
\hline 6.4 & 3.0 & -10.6 & $2.2 \times 10^{-13}$ & $4.7 \times 10^{-9}$ & ANPEP & Alanyl (membrane) aminopeptidase \\
\hline 6.7 & 3.8 & -7.7 & $4.4 \times 10^{-13}$ & $4.7 \times 10^{-9}$ & EPS8L3 & EPS8-like 3 \\
\hline 7.0 & 4.1 & -7.4 & $1.2 \times 10^{-12}$ & $7.1 \times 10^{-9}$ & SLC44A4 & Solute carrier family 44 , member 4 \\
\hline 7.6 & 4.7 & -7.3 & $1.0 \times 10^{-4}$ & $1.2 \times 10^{-2}$ & BGN & Biglycan \\
\hline 7.5 & 4.8 & -6.3 & $1.3 \times 10^{-11}$ & $3.4 \times 10^{-8}$ & LDHB & Lactate dehydrogenase B \\
\hline 6.2 & 3.6 & -6.3 & $2.5 \times 10^{-11}$ & $5.4 \times 10^{-8}$ & CPNE2 & Copine II \\
\hline 5.7 & 3.2 & -5.7 & $1.7 \times 10^{-12}$ & $9.0 \times 10^{-9}$ & $\mathrm{DSC} 3$ & Desmocollin 3 \\
\hline 5.7 & 3.2 & -5.6 & $8.2 \times 10^{-7}$ & $2.0 \times 10^{-4}$ & CRYBB1 & Crystallin beta B1 \\
\hline 5.8 & 3.3 & -5.4 & $1.2 \times 10^{-9}$ & $1.4 \times 10^{-6}$ & AKR1C2 & Aldo-keto reductase family 1 , member $\mathrm{C} 2$ \\
\hline 7.6 & 5.4 & -4.7 & $1.9 \times 10^{-13}$ & $4.7 \times 10^{-9}$ & AKR1B10 & Aldo-keto reductase family 1 , member B10 \\
\hline 5.3 & 3.1 & -4.5 & $9.9 \times 10^{-6}$ & $1.6 \times 10^{-3}$ & LINC00942 & Long intergenic non-protein coding RNA 942 \\
\hline 6.5 & 4.5 & -4.1 & $5.6 \times 10^{-11}$ & $1.1 \times 10^{-7}$ & SLC9A2 & Solute carrier family 9 , subfamily A \\
\hline 6.6 & 4.6 & -4.0 & $4.4 \times 10^{-9}$ & $4.1 \times 10^{-6}$ & LDHA & Lactate dehydrogenase A \\
\hline 6.0 & 4.0 & -4.0 & $1.1 \times 10^{-7}$ & $4.6 \times 10^{-5}$ & GAL & Galanin/GMAP prepropeptide \\
\hline 5.6 & 3.6 & -3.9 & $5.0 \times 10^{-11}$ & $1.0 \times 10^{-7}$ & WWC2 & WW and $\mathrm{C} 2$ domain containing 2 \\
\hline 8.2 & 6.2 & -3.9 & $1.2 \times 10^{-2}$ & $1.8 \times 10^{-1}$ & CYP1A2 & Cytochrome P450, family 1 , sf A, polypeptide 2 \\
\hline 5.2 & 3.2 & -3.8 & $1.7 \times 10^{-6}$ & $4.0 \times 10^{-4}$ & HULC & H.C up-regulated long non-coding RNA \\
\hline 6.3 & 4.3 & -3.8 & $6.3 \times 10^{-10}$ & $8.2 \times 10^{-7}$ & $\mathrm{ABCC} 2$ & ATP binding cassette subfamily $\mathrm{C}$ member 2 \\
\hline 7.9 & 6.1 & -3.5 & $2.9 \times 10^{-12}$ & $1.0 \times 10^{-8}$ & MSN & Moesin \\
\hline 5.1 & 3.3 & -3.5 & $2.8 \times 10^{-10}$ & $4.3 \times 10^{-7}$ & BCL11A & B-cell CLL/lymphoma 11A (zinc finger protein) \\
\hline 4.5 & 2.7 & -3.4 & $6.0 \times 10^{-5}$ & $6.1 \times 10^{-3}$ & NOX1 & NADPH oxidase 1 \\
\hline 4.9 & 3.1 & -3.4 & $3.5 \times 10^{-6}$ & $7.0 \times 10^{-4}$ & HUNK & Hormonally up-regulated Neu-associated kinase \\
\hline 6.8 & 5.1 & -3.4 & $2.4 \times 10^{-2}$ & $2.6 \times 10^{-1}$ & $\mathrm{CHAC} 1$ & $\begin{array}{l}\text { ChaC glutathionx } 10 \text {-specific } \\
\text { gamma-glutamylcyclotransferase } 1\end{array}$ \\
\hline 5.7 & 4.0 & -3.3 & $7.0 \times 10^{-9}$ & $5.7 \times 10^{-6}$ & THSD4 & Thrombospondin type 1 domain containing 4 \\
\hline 7.3 & 5.6 & -3.3 & $2.1 \times 10^{-9}$ & $2.2 \times 10^{-6}$ & $\mathrm{CDH} 3$ & Cadherin 3 , type 1, P-cadherin (placental) \\
\hline 7.2 & 5.5 & -3.2 & $3.7 \times 10^{-7}$ & $1.0 \times 10^{-4}$ & ETV4 & Ets variant 4 \\
\hline 5.3 & 3.6 & -3.2 & $1.4 \times 10^{-5}$ & $2.0 \times 10^{-3}$ & HBE1 & Hemoglobin, epsilon 1 \\
\hline 5.5 & 3.8 & -3.2 & $9.1 \times 10^{-9}$ & $7.0 \times 10^{-6}$ & CLIC3 & Chloride intracellular channel 3 \\
\hline 5.2 & 3.6 & -3.1 & $2.9 \times 10^{-7}$ & $1.0 \times 10^{-4}$ & IGFBP2 & Insulin like growth factor binding protein 2 \\
\hline 7.1 & 5.4 & -3.1 & $2.9 \times 10^{-5}$ & $3.6 \times 10^{-3}$ & CALB2 & Calbindin 2 \\
\hline 8.3 & 6.7 & -3.0 & $3.7 \times 10^{-6}$ & $8.0 \times 10^{-4}$ & ALDH3A1 & Aldehyde dehydrogenase 3 family, member A1 \\
\hline 5.4 & 3.8 & -3.0 & $5.8 \times 10^{-7}$ & $2.0 \times 10^{-4}$ & CAMK2N1 & Calcium/calmodulin-dependent protein kinase II inhibitor 1 \\
\hline 6.2 & 4.6 & -2.9 & $6.1 \times 10^{-9}$ & $5.2 \times 10^{-6}$ & PTPN18 & $\begin{array}{c}\text { Protein tyrosine phosphatase, non-receptor } \\
\text { type } 18 \text { (brain-derived) }\end{array}$ \\
\hline 4.3 & 2.8 & -2.9 & $1.7 \times 10^{-8}$ & $1.1 \times 10^{-5}$ & EDARADD & EDAR-associated death domain \\
\hline 6.1 & 4.6 & -2.9 & $5.4 \times 10^{-7}$ & $2.0 \times 10^{-4}$ & FGFBP1 & Fibroblast growth factor binding protein 1 \\
\hline 4.1 & 2.7 & -2.8 & $6.7 \times 10^{-8}$ & $3.2 \times 10^{-5}$ & SPIN3 & Spindlin family, member 3 \\
\hline 5.6 & 4.2 & -2.8 & $4.8 \times 10^{-6}$ & $9.0 \times 10^{-4}$ & $\mathrm{CORO} 2 \mathrm{~A}$ & Coronin, actin binding protein, $2 \mathrm{~A}$ \\
\hline 4.1 & 2.7 & -2.7 & $7.0 \times 10^{-4}$ & $3.4 \times 10^{-2}$ & OR51B4 & Olfactory receptor, family 51 , subfamily $B$, member 4 \\
\hline 5.8 & 4.4 & -2.7 & $6.0 \times 10^{-4}$ & $2.9 \times 10^{-2}$ & EIF2B5-AS1 & EIF2B5 antisense RNA 1 \\
\hline 8.2 & 6.7 & -2.7 & $1.5 \times 10^{-5}$ & $2.2 \times 10^{-3}$ & GPX2 & Glutathione peroxidase 2 \\
\hline 2.9 & 1.5 & -2.7 & $6.3 \times 10^{-7}$ & $2.0 \times 10^{-4}$ & GPX8 & Glutathione peroxidase 8 (putative) \\
\hline 4.7 & 3.2 & -2.7 & $5.3 \times 10^{-9}$ & $4.6 \times 10^{-6}$ & ANOS1 & Anosmin 1 \\
\hline 10.3 & 8.9 & -2.7 & $8.5 \times 10^{-6}$ & $1.4 \times 10^{-3}$ & SLC7A11 & $\begin{array}{l}\text { Solute carrier family } 7 \text { (anionic amino acid } \\
\text { transporter light chain, xc- system), member } 11\end{array}$ \\
\hline 6.3 & 4.8 & -2.7 & $9.7 \times 10^{-3}$ & $1.6 \times 10^{-1}$ & VCAN & Versican \\
\hline 5.9 & 4.5 & -2.7 & $1.5 \times 10^{-6}$ & $4.0 \times 10^{-4}$ & SLPI & Secretory leukocyte peptidase inhibitor \\
\hline 6.1 & 4.7 & -2.7 & $2.6 \times 10^{-9}$ & $2.7 \times 10^{-6}$ & TFCP2 & Transcription factor $\mathrm{CP} 2$ \\
\hline 5.8 & 4.4 & -2.7 & $8.0 \times 10^{-8}$ & $3.7 \times 10^{-5}$ & DNM1 & Dynamin 1 \\
\hline 5.0 & 3.5 & -2.6 & $9.3 \times 10^{-3}$ & $1.6 \times 10^{-1}$ & IL37 & Interleukin 37 \\
\hline 4.8 & 3.4 & -2.6 & $3.4 \times 10^{-8}$ & $1.9 \times 10^{-5}$ & ANO1 & Anoctamin 1 , calcium activated chloride channel \\
\hline 7.2 & 5.8 & -2.6 & $4.6 \times 10^{-11}$ & $9.7 \times 10^{-8}$ & CA12 & Carbonic anhydrase XII \\
\hline 5.8 & 4.4 & -2.6 & $5.5 \times 10^{-6}$ & $1.0 \times 10^{-3}$ & CES1; CES1P1 & Carboxylesterase 1 ; carboxylesterase 1 pseudogene 1 \\
\hline 4.2 & 2.8 & -2.6 & $1.1 \times 10^{-8}$ & $7.8 \times 10^{-6}$ & ALDH1A1 & Aldehyde dehydrogenase 1 family, member A1 \\
\hline 4.2 & 2.8 & -2.6 & $1.8 \times 10^{-8}$ & $1.2 \times 10^{-5}$ & LYZ & Lysozyme \\
\hline
\end{tabular}

Table I. Continued 
Table I. Continued

\begin{tabular}{|c|c|c|c|c|c|c|}
\hline $\begin{array}{l}\text { Control WT } \\
\text { Avg }(\log 2)\end{array}$ & $\begin{array}{l}\text { LDHA/B DKO } \\
\text { Avg }(\log 2)\end{array}$ & $\begin{array}{l}\text { Fold } \\
\text { change }\end{array}$ & $p$-Value & $\begin{array}{c}\text { FDR } \\
p \text {-Value }\end{array}$ & $\begin{array}{l}\text { Gene } \\
\text { symbol }\end{array}$ & Description \\
\hline 6.3 & 4.9 & -2.5 & $4.6 \times 10^{-9}$ & $4.1 \times 10^{-6}$ & LCN2 & Lipocalin 2 \\
\hline 4.9 & 3.6 & -2.5 & $2.3 \times 10^{-8}$ & $1.5 \times 10^{-5}$ & TCEA3 & Transcription elongation factor A (SII), 3 \\
\hline 4.7 & 3.3 & -2.5 & $1.6 \times 10^{-8}$ & $1.1 \times 10^{-5}$ & GREB1L & Growth regulation by estrogen in breast cancer-like \\
\hline 7.9 & 6.6 & -2.5 & $3.4 \times 10^{-9}$ & $3.4 \times 10^{-6}$ & CLDN3 & Claudin 3 \\
\hline 4.6 & 3.3 & -2.5 & $8.4 \times 10^{-6}$ & $1.4 \times 10^{-3}$ & TET1 & Tet methylcytosine dioxygenase 1 \\
\hline 6.8 & 5.6 & -2.5 & $4.7 \times 10^{-3}$ & $1.1 \times 10^{-1}$ & LCN15 & Lipocalin 15 \\
\hline 5.3 & 4.0 & -2.5 & $3.2 \times 10^{-6}$ & $7.0 \times 10^{-4}$ & SOD3 & Superoxide dismutase 3 , extracellular \\
\hline 4.9 & 3.6 & -2.4 & $1.2 \times 10^{-9}$ & $1.4 \times 10^{-6}$ & FXYD5 & FXYD domain containing ion transport regulator 5 \\
\hline 7.1 & 5.9 & -2.4 & $2.5 \times 10^{-8}$ & $1.5 \times 10^{-5}$ & SLC44A2 & Solute carrier family 44 (choline transporter), member 2 \\
\hline 3.5 & 2.3 & -2.4 & $3.9 \times 10^{-8}$ & $2.1 \times 10^{-5}$ & TSPAN7 & Tetraspanin 7 \\
\hline 3.7 & 2.4 & -2.4 & $6.0 \times 10^{-4}$ & $3.2 \times 10^{-2}$ & TREX2 & Three prime repair exonuclease 2 \\
\hline 6.0 & 4.7 & -2.3 & $3.6 \times 10^{-9}$ & $3.5 \times 10^{-6}$ & FGF19 & Fibroblast growth factor 19 \\
\hline 3.8 & 2.6 & -2.3 & $1.3 \times 10^{-5}$ & $1.9 \times 10^{-3}$ & SRGAP1 & SLIT-ROBO Rho GTPase activating protein 1 \\
\hline 3.9 & 2.7 & -2.3 & $9.8 \times 10^{-5}$ & $8.8 \times 10^{-3}$ & ALDH2 & Aldehyde dehydrogenase 2 family (mitochondrial) \\
\hline 5.4 & 4.2 & -2.3 & $3.9 \times 10^{-6}$ & $8.0 \times 10^{-4}$ & GCNT3 & Glucosaminyl (N-acetyl) transferase 3 , mucin type \\
\hline 6.2 & 5.0 & -2.3 & $1.3 \times 10^{-7}$ & $5.4 \times 10^{-5}$ & PFKP & Phosphofructokinase, platelet \\
\hline 3.9 & 2.7 & -2.3 & $1.8 \times 10^{-5}$ & $2.5 \times 10^{-3}$ & IL1A & Interleukin 1 alpha \\
\hline 6.8 & 5.6 & -2.3 & $3.5 \times 10^{-2}$ & $3.1 \times 10^{-1}$ & $\mathrm{CTH}$ & Cystathionine gamma-lyase \\
\hline 6.8 & 5.6 & -2.3 & $2.6 \times 10^{-6}$ & $6.0 \times 10^{-4}$ & RCN1 & Reticulocalbin 1, EF-hand calcium binding domain \\
\hline 9.4 & 8.2 & -2.3 & $4.1 \times 10^{-5}$ & $4.7 \times 10^{-3}$ & ASS1 & Argininosuccinate synthase 1 \\
\hline 5.1 & 4.0 & -2.2 & $3.3 \times 10^{-5}$ & $3.9 \times 10^{-3}$ & SYNE4 & $\begin{array}{l}\text { Spectrin repeat containing, nuclear envelope } \\
\text { family member } 4\end{array}$ \\
\hline 4.2 & 3.1 & -2.2 & $6.3 \times 10^{-8}$ & $3.1 \times 10^{-5}$ & TRIM7 & Tripartite motif containing 7 \\
\hline 6.0 & 4.9 & -2.2 & $2.6 \times 10^{-7}$ & $9.2 \times 10^{-5}$ & PAH & Phenylalanine hydroxylase \\
\hline 5.0 & 3.8 & -2.2 & $6.3 \times 10^{-6}$ & $1.1 \times 10^{-3}$ & SLC16A5 & $\begin{array}{l}\text { Solute carrier family } 16 \\
\text { (monocarboxylate transporter), member } 5\end{array}$ \\
\hline 5.1 & 4.0 & -2.2 & $6.7 \times 10^{-7}$ & $2.0 \times 10^{-4}$ & LAMB2 & Laminin, beta 2 (laminin S) \\
\hline 5.0 & 3.9 & -2.2 & $5.0 \times 10^{-4}$ & $2.6 \times 10^{-2}$ & ATP9A & ATPase, class II, type 9A \\
\hline 5.4 & 4.3 & -2.2 & $1.4 \times 10^{-5}$ & $2.0 \times 10^{-3}$ & ABAT & 4-aminobutyrate aminotransferase \\
\hline 6.5 & 5.4 & -2.2 & $1.6 \times 10^{-2}$ & $2.1 \times 10^{-1}$ & $\mathrm{ATP} 2 \mathrm{C} 2$ & ATPase, $\mathrm{Ca}++$ transporting, type $2 \mathrm{C}$, member 2 \\
\hline 5.6 & 4.5 & -2.2 & $9.8 \times 10^{-8}$ & $4.3 \times 10^{-5}$ & EFNA3 & Ephrin-A3 \\
\hline 5.9 & 4.8 & -2.2 & $1.0 \times 10^{-4}$ & $9.6 \times 10^{-3}$ & CORO1A & Coronin, actin binding protein, $1 \mathrm{~A}$ \\
\hline 5.9 & 4.8 & -2.2 & $7.0 \times 10^{-9}$ & $5.7 \times 10^{-6}$ & PLA2G16 & Phospholipase A2, group XVI \\
\hline 4.5 & 3.4 & -2.2 & $4.4 \times 10^{-5}$ & $4.9 \times 10^{-3}$ & PTPRU & Protein tyrosine phosphatase, receptor type, $\mathrm{U}$ \\
\hline 4.0 & 2.9 & -2.2 & $3.8 \times 10^{-7}$ & $1.0 \times 10^{-4}$ & SPATA6L & Spermatogenesis associated 6-like \\
\hline 5.1 & 4.0 & -2.1 & $2.0 \times 10^{-4}$ & $1.3 \times 10^{-2}$ & $\begin{array}{l}\text { SNORA11; } \\
\text { MAGED2 }\end{array}$ & $\begin{array}{l}\text { Small nucleolar RNA, H/ACA box } 11 ; \\
\text { MAGE family member D2 }\end{array}$ \\
\hline 5.0 & 3.9 & -2.1 & $6.4 \times 10^{-6}$ & $1.1 \times 10^{-3}$ & AKR1C1 & Aldo-keto reductase family 1 , member $\mathrm{C} 1$ \\
\hline 6.3 & 5.2 & -2.1 & $2.3 \times 10^{-7}$ & $8.6 \times 10^{-5}$ & FUOM & Fucose mutarotase \\
\hline 5.2 & 4.2 & -2.1 & $2.4 \times 10^{-9}$ & $2.5 \times 10^{-6}$ & CYP2J2 & Cytochrome P450, family 2, subfamily J, polypeptide 2 \\
\hline 5.2 & 4.1 & -2.1 & $6.1 \times 10^{-5}$ & $6.2 \times 10^{-3}$ & CAPN5 & Calpain 5 \\
\hline 7.3 & 6.2 & -2.1 & $5.2 \times 10^{-6}$ & $1.0 \times 10^{-3}$ & HOXB9 & Homeobox B9 \\
\hline 4.0 & 2.9 & -2.1 & $9.5 \times 10^{-3}$ & $1.6 \times 10^{-1}$ & MIR1206 & MicroRNA 1206 \\
\hline 5.9 & 4.9 & -2.1 & $4.6 \times 10^{-8}$ & $2.4 \times 10^{-5}$ & KIZ & Kizuna centrosomal protein \\
\hline 6.3 & 5.2 & -2.1 & $1.4 \times 10^{-7}$ & $5.7 \times 10^{-5}$ & SERPINB5 & $\begin{array}{l}\text { Serpin peptidase inhibitor, clade B } \\
\text { (ovalbumin), member } 5\end{array}$ \\
\hline 5.9 & 4.8 & -2.1 & $2.7 \times 10^{-2}$ & $2.7 \times 10^{-1}$ & SLC6A9 & $\begin{array}{l}\text { Solute carrier family } 6 \\
\text { (neurotransmitter transporter, glycine), member } 9\end{array}$ \\
\hline 6.9 & 5.9 & -2.1 & $2.1 \times 10^{-2}$ & $2.4 \times 10^{-1}$ & STC2 & Stanniocalcin 2 \\
\hline 4.4 & 3.4 & -2.1 & $4.8 \times 10^{-6}$ & $9.0 \times 10^{-4}$ & $\begin{array}{l}\text { LEMD1; } \\
\text { BLACAT1 }\end{array}$ & $\begin{array}{l}\text { LEM domain containing } 1 \text {; bladder cancer } \\
\text { associated transcript } 1 \text { (non-protein coding) }\end{array}$ \\
\hline 5.0 & 4.0 & -2.1 & $1.3 \times 10^{-5}$ & $1.9 \times 10^{-3}$ & MYH13 & Myosin, heavy chain 13 , skeletal muscle \\
\hline 4.5 & 3.5 & -2.1 & $1.5 \times 10^{-6}$ & $4.0 \times 10^{-4}$ & SLC34A3 & $\begin{array}{l}\text { Solute carrier family } 34 \text { (type II sodium/ } \\
\text { phosphate cotransporter), member } 3\end{array}$ \\
\hline 4.4 & 3.4 & -2.1 & $2.4 \times 10^{-5}$ & $3.1 \times 10^{-3}$ & $\begin{array}{l}\text { AIFM3; } \\
\text { LZTR1 }\end{array}$ & $\begin{array}{l}\text { Apoptosis-inducing factor, mitochondrion-associated, } \\
\text { 3; leucinx10-zipper-like transcription regulator } 1\end{array}$ \\
\hline 5.2 & 4.2 & -2.1 & $1.8 \times 10^{-5}$ & $2.5 \times 10^{-3}$ & CRYBA4 & Crystallin beta A4 \\
\hline 4.5 & 3.4 & -2.1 & $4.0 \times 10^{-4}$ & $2.3 \times 10^{-2}$ & INAFM2 & InaF-motif containing 2 \\
\hline
\end{tabular}

Table I. Continued 
Table I. Continued

\begin{tabular}{|c|c|c|c|c|c|c|}
\hline $\begin{array}{l}\text { Control WT } \\
\text { Avg }(\log 2)\end{array}$ & $\begin{array}{l}\text { LDHA/B DKO } \\
\text { Avg }(\log 2)\end{array}$ & $\begin{array}{l}\text { Fold } \\
\text { change }\end{array}$ & $p$-Value & $\begin{array}{c}\text { FDR } \\
p \text {-Value }\end{array}$ & $\begin{array}{l}\text { Gene } \\
\text { symbol }\end{array}$ & Description \\
\hline 2.9 & 1.8 & -2.1 & $1.7 \times 10^{-6}$ & $4.0 \times 10^{-4}$ & $\begin{array}{l}\text { OR51B5; } \\
\text { HBG2; } \\
\text { HBE1 }\end{array}$ & $\begin{array}{c}\text { Olfactory receptor, family } 51 \text {, subfamily } \mathrm{B}, \\
\text { member } 5 \text {; hemoglobin, gamma G; } \\
\text { hemoglobin, epsilon } 1\end{array}$ \\
\hline 5.6 & 4.6 & -2.1 & $8.2 \times 10^{-8}$ & $3.8 \times 10^{-5}$ & DMTN & Dematin actin binding protein \\
\hline 6.9 & 5.9 & -2.1 & $3.7 \times 10^{-8}$ & $2.0 \times 10^{-5}$ & PLLP & Plasmolipin \\
\hline 3.4 & 2.3 & -2.1 & $2.1 \times 10^{-6}$ & $5.0 \times 10^{-4}$ & GXYLT2 & Glucoside xylosyltransferase 2 \\
\hline 6.2 & 5.2 & -2.0 & $9.7 \times 10^{-5}$ & $8.7 \times 10^{-3}$ & LIF & Leukemia inhibitory factor \\
\hline 2.5 & 1.5 & -2.0 & $8.0 \times 10^{-3}$ & $1.5 \times 10^{-1}$ & MIR3646 & MicroRNA 3646 \\
\hline 7.2 & 6.2 & -2.0 & $2.0 \times 10^{-9}$ & $2.2 \times 10^{-6}$ & TMEM214 & Transmembrane protein 214 \\
\hline 5.1 & 4.0 & -2.0 & $1.9 \times 10^{-8}$ & $1.2 \times 10^{-5}$ & TPST2 & Tyrosylprotein sulfotransferase 2 \\
\hline 6.2 & 5.2 & -2.0 & $7.3 \times 10^{-6}$ & $1.3 \times 10^{-3}$ & APCDD1 & Adenomatosis polyposis coli down-regulated 1 \\
\hline 6.4 & 5.4 & -2.0 & $1.0 \times 10^{-4}$ & $9.2 \times 10^{-3}$ & COTL1 & Coactosin-like F-actin binding protein 1 \\
\hline 3.1 & 4.1 & 2.0 & $6.8 \times 10^{-5}$ & $6.8 \times 10^{-3}$ & CXCL10 & Chemokine (C-X-C motif) ligand 10 \\
\hline 3.3 & 4.3 & 2.0 & $1.6 \times 10^{-2}$ & $2.1 \times 10^{-1}$ & $\begin{array}{l}\text { SCARNA15; } \\
\text { SNHG21 }\end{array}$ & $\begin{array}{l}\text { Small Cajal body-specific RNA } 15 \\
\text { small nucleolar RNA host gene } 21\end{array}$ \\
\hline 8.1 & 9.1 & 2.0 & $4.4 \times 10^{-8}$ & $2.3 \times 10^{-5}$ & TXNIP & Thioredoxin interacting protein \\
\hline 3.4 & 4.4 & 2.0 & $6.0 \times 10^{-4}$ & $3.1 \times 10^{-2}$ & TOX3 & TOX high mobility group box family member 3 \\
\hline 3.7 & 4.7 & 2.0 & $9.3 \times 10^{-6}$ & $1.5 \times 10^{-3}$ & C2orf54 & Chromosome 2 open reading frame 54 \\
\hline 6.0 & 7.0 & 2.0 & $1.4 \times 10^{-8}$ & $1.0 \times 10^{-5}$ & ISG15 & ISG15 ubiquitin-like modifier \\
\hline 3.9 & 4.9 & 2.0 & $1.6 \times 10^{-6}$ & $4.0 \times 10^{-4}$ & FAM230B & $\begin{array}{l}\text { Family with sequence similarity } 230, \\
\text { member B (non-protein coding) }\end{array}$ \\
\hline 3.7 & 4.7 & 2.0 & $4.0 \times 10^{-4}$ & $2.2 \times 10^{-2}$ & IFI16 & Interferon, gamma-inducible protein 16 \\
\hline 2.9 & 3.9 & 2.0 & $2.6 \times 10^{-3}$ & $7.8 \times 10^{-2}$ & LYST & Lysosomal trafficking regulator \\
\hline 4.4 & 5.4 & 2.1 & $3.9 \times 10^{-5}$ & $4.5 \times 10^{-3}$ & IFIH1 & Interferon induced, with helicase $\mathrm{C}$ domain 1 \\
\hline 3.7 & 4.7 & 2.1 & $1.1 \times 10^{-2}$ & $1.7 \times 10^{-1}$ & RNU1-13P & RNA, U1 small nuclear 13 , pseudogene \\
\hline 3.7 & 4.7 & 2.1 & $1.1 \times 10^{-2}$ & $1.7 \times 10^{-1}$ & RNU1-13P & RNA, U1 small nuclear 13, pseudogene \\
\hline 6.2 & 7.3 & 2.1 & $3.0 \times 10^{-6}$ & $6.0 \times 10^{-4}$ & ARRDC4 & Arrestin domain containing 4 \\
\hline 4.1 & 5.1 & 2.1 & $2.5 \times 10^{-8}$ & $1.5 \times 10^{-5}$ & CHST15 & $\begin{array}{l}\text { Carbohydrate }(\mathrm{N} \text {-acetylgalactosamine } \\
\text { 4-sulfate } 6-\mathrm{O}) \text { sulfotransferase } 15\end{array}$ \\
\hline 3.6 & 4.7 & 2.1 & $1.1 \times 10^{-2}$ & $1.8 \times 10^{-1}$ & AGR3 & $\begin{array}{l}\text { Anterior gradient } 3 \text {, protein disulphide } \\
\text { isomerase family member }\end{array}$ \\
\hline 3.3 & 4.3 & 2.1 & $7.3 \times 10^{-3}$ & $1.4 \times 10^{-1}$ & MIR3620; ARF1 & MicroRNA 3620; ADP-ribosylation factor 1 \\
\hline 3.2 & 4.2 & 2.1 & $2.2 \times 10^{-5}$ & $2.9 \times 10^{-3}$ & MIR3622A & MicroRNA 3622a \\
\hline 6.4 & 7.5 & 2.1 & $3.0 \times 10^{-4}$ & $1.9 \times 10^{-2}$ & SCARNA4 & Small Cajal body-specific RNA 4 \\
\hline 4.6 & 5.7 & 2.2 & $5.2 \times 10^{-5}$ & $5.6 \times 10^{-3}$ & OASL & 2-5-oligoadenylate synthetasx 10 -like \\
\hline 1.9 & 3.0 & 2.2 & $7.0 \times 10^{-4}$ & $3.5 \times 10^{-2}$ & MIR4727 & MicroRNA 4727 \\
\hline 5.7 & 6.8 & 2.2 & $1.8 \times 10^{-5}$ & $2.5 \times 10^{-3}$ & SECTM1 & Secreted and transmembrane 1 \\
\hline 4.5 & 5.7 & 2.2 & $4.5 \times 10^{-9}$ & $4.1 \times 10^{-6}$ & SATB1 & SATB homeobox 1 \\
\hline 4.4 & 5.5 & 2.2 & $1.3 \times 10^{-2}$ & $1.9 \times 10^{-1}$ & SCARNA3 & Small Cajal body-specific RNA 3 \\
\hline 6.4 & 7.6 & 2.2 & $2.0 \times 10^{-4}$ & $1.2 \times 10^{-2}$ & XAF1 & XIAP associated factor 1 \\
\hline 3.6 & 4.8 & 2.3 & $7.4 \times 10^{-3}$ & $1.4 \times 10^{-1}$ & ARMC3 & Armadillo repeat containing 3 \\
\hline 5.9 & 7.1 & 2.3 & $1.4 \times 10^{-5}$ & $2.1 \times 10^{-3}$ & LYST & Lysosomal trafficking regulator \\
\hline 2.9 & 4.1 & 2.3 & $3.0 \times 10^{-4}$ & $2.0 \times 10^{-2}$ & C6orf222 & Chromosome 6 open reading frame 222 \\
\hline 7.9 & 9.2 & 2.4 & $9.0 \times 10^{-8}$ & $4.1 \times 10^{-5}$ & GPR137B & G protein-coupled receptor 137B \\
\hline 4.0 & 5.3 & 2.4 & $6.4 \times 10^{-7}$ & $2.0 \times 10^{-4}$ & DUSP10 & Dual specificity phosphatase 10 \\
\hline 3.1 & 4.3 & 2.4 & $8.9 \times 10^{-5}$ & $8.2 \times 10^{-3}$ & LDB2 & LIM domain binding 2 \\
\hline 8.5 & 9.8 & 2.4 & $1.0 \times 10^{-2}$ & $1.7 \times 10^{-1}$ & SNORA23 & Small nucleolar RNA, H/ACA box 23 \\
\hline 6.7 & 8.0 & 2.4 & $5.4 \times 10^{-6}$ & $1.0 \times 10^{-3}$ & HMGCS2 & $\begin{array}{l}\text { 3-hydroxy-3-methylglutaryl-CoA } \\
\text { synthase } 2 \text { (mitochondrial) }\end{array}$ \\
\hline 5.3 & 6.6 & 2.4 & $9.5 \times 10^{-6}$ & $1.5 \times 10^{-3}$ & PLSCR 1 & Phospholipid scramblase 1 \\
\hline 6.4 & 7.7 & 2.5 & $2.3 \times 10^{-7}$ & $8.6 \times 10^{-5}$ & HERC6 & $\begin{array}{l}\text { HECT and RLD domain containing E3 } \\
\text { ubiquitin protein ligase family member } 6\end{array}$ \\
\hline 3.6 & 4.9 & 2.5 & $1.7 \times 10^{-8}$ & $1.1 \times 10^{-5}$ & ALOX12P2 & Arachidonate 12-lipoxygenase pseudogene 2 \\
\hline 1.1 & 2.5 & 2.5 & $2.5 \times 10^{-2}$ & $2.6 \times 10^{-1}$ & MIR642B & MicroRNA $642 \mathrm{~b}$ \\
\hline 2.1 & 3.4 & 2.5 & $1.7 \times 10^{-3}$ & $5.9 \times 10^{-2}$ & KRTAP10-1 & Keratin associated protein $10-1$ \\
\hline 1.6 & 3.0 & 2.5 & $1.1 \times 10^{-5}$ & $1.7 \times 10^{-3}$ & OR51C1P & $\begin{array}{l}\text { Olfactory receptor, family } 51 \text {, subfamily } \mathrm{C}, \\
\text { member } 1 \text { pseudogene }\end{array}$ \\
\hline 5.5 & 6.8 & 2.5 & $1.0 \times 10^{-6}$ & $3.0 \times 10^{-4}$ & IFI27 & Interferon, alpha-inducible protein 27 \\
\hline
\end{tabular}

Table I. Continued 
Table I. Continued

\begin{tabular}{|c|c|c|c|c|c|c|}
\hline $\begin{array}{l}\text { Control WT } \\
\text { Avg }(\log 2)\end{array}$ & $\begin{array}{l}\text { LDHA/B DKO } \\
\text { Avg }(\log 2)\end{array}$ & $\begin{array}{l}\text { Fold } \\
\text { change }\end{array}$ & $p$-Value & $\begin{array}{c}\text { FDR } \\
p \text {-Value }\end{array}$ & $\begin{array}{l}\text { Gene } \\
\text { symbol }\end{array}$ & Description \\
\hline 3.3 & 4.6 & 2.5 & $3.6 \times 10^{-8}$ & $2.0 \times 10^{-5}$ & ZSCAN12P1 & $\begin{array}{l}\text { Zinc finger and SCAN domain } \\
\text { containing } 12 \text { pseudogene } 1\end{array}$ \\
\hline 2.1 & 3.5 & 2.6 & $3.0 \times 10^{-8}$ & $1.7 \times 10^{-5}$ & UBE2U & Ubiquitin-conjugating enzyme E2U (putative) \\
\hline 5.1 & 6.4 & 2.6 & $5.2 \times 10^{-10}$ & $7.2 \times 10^{-7}$ & SEMA6A & $\begin{array}{l}\text { Sema domain, transmembrane domain } \\
(\mathrm{TM}) \text {, and cytoplasmic domain, (semaphorin) } 6 \mathrm{~A}\end{array}$ \\
\hline 8.0 & 9.4 & 2.6 & $7.0 \times 10^{-11}$ & $1.3 \times 10^{-7}$ & SMOC2 & SPARC related modular calcium binding 2 \\
\hline 5.1 & 6.5 & 2.7 & $3.8 \times 10^{-8}$ & $2.1 \times 10^{-5}$ & SAMD9 & Sterile alpha motif domain containing 9 \\
\hline 6.5 & 7.9 & 2.7 & $1.1 \times 10^{-7}$ & $4.7 \times 10^{-5}$ & SNORA75 & Small nucleolar RNA, H/ACA box 75 \\
\hline 4.0 & 5.4 & 2.7 & $7.6 \times 10^{-6}$ & $1.3 \times 10^{-3}$ & NR0B2 & Nuclear receptor subfamily 0 , group B, member 2 \\
\hline 2.5 & 3.9 & 2.7 & $2.6 \times 10^{-8}$ & $1.5 \times 10^{-5}$ & $\begin{array}{l}\text { LOC } 101927859 ; \\
\text { LOC105369185; } \\
\text { LOC105372941 }\end{array}$ & $\begin{array}{l}\text { Serine/arginine repetitive matrix protein 2-like; } \\
\text { uncharacterized LOC105369185; } \\
\text { uncharacterized LOC105372941 }\end{array}$ \\
\hline 5.1 & 6.5 & 2.7 & $4.0 \times 10^{-4}$ & $2.5 \times 10^{-2}$ & PCK1 & Phosphoenolpyruvate carboxykinase 1 (soluble) \\
\hline 3.5 & 5.0 & 2.7 & $3.3 \times 10^{-7}$ & $1.0 \times 10^{-4}$ & NPC1L1 & NPC1-like 1 \\
\hline 2.8 & 4.3 & 2.8 & $1.0 \times 10^{-2}$ & $1.7 \times 10^{-1}$ & MIR4659A & MicroRNA 4659a \\
\hline 4.3 & 5.8 & 2.8 & $6.2 \times 10^{-9}$ & $5.3 \times 10^{-6}$ & SAMD9L & Sterile alpha motif domain containing 9-like \\
\hline 4.8 & 6.4 & 2.9 & $1.9 \times 10^{-5}$ & $2.5 \times 10^{-3}$ & LINC00520 & Long intergenic non-protein coding RNA 520 \\
\hline 2.8 & 4.3 & 2.9 & $1.6 \times 10^{-10}$ & $2.8 \times 10^{-7}$ & CSF2RA & $\begin{array}{l}\text { Colony stimulating factor } 2 \text { receptor, alpha, } \\
\text { low-affinity (granulocytx 10-macrophage) }\end{array}$ \\
\hline 3.0 & 4.6 & 3.0 & $8.4 \times 10^{-9}$ & $6.5 \times 10^{-6}$ & ASB4 & Ankyrin repeat and SOCS box containing 4 \\
\hline 5.2 & 6.8 & 3.0 & $1.7 \times 10^{-8}$ & $1.1 \times 10^{-5}$ & ENC1 & Ectodermal-neural cortex 1 (with BTB domain) \\
\hline 2.8 & 4.4 & 3.2 & $4.2 \times 10^{-2}$ & $3.4 \times 10^{-1}$ & SNORA2A & Small nucleolar RNA, H/ACA box 2A \\
\hline 5.6 & 7.3 & 3.2 & $3.5 \times 10^{-7}$ & $1.0 \times 10^{-4}$ & DDX58 & DEAD (Asp-Glu-Ala-Asp) box polypeptide 58 \\
\hline 2.5 & 4.2 & 3.2 & $2.3 \times 10^{-8}$ & $1.5 \times 10^{-5}$ & TUSC3 & Tumor suppressor candidate 3 \\
\hline 6.7 & 8.4 & 3.3 & $9.2 \times 10^{-8}$ & $4.2 \times 10^{-5}$ & CD68 & CD68 molecule \\
\hline 4.3 & 6.0 & 3.4 & $6.7 \times 10^{-10}$ & $8.5 \times 10^{-7}$ & PADI1 & Peptidyl arginine deiminase, type I \\
\hline 3.9 & 5.7 & 3.4 & $3.8 \times 10^{-10}$ & $5.4 \times 10^{-7}$ & PPARGC1A & $\begin{array}{l}\text { Peroxisome proliferator-activated } \\
\text { receptor gamma, coactivator } 1 \text { alpha }\end{array}$ \\
\hline 4.8 & 6.7 & 3.7 & $3.0 \times 10^{-10}$ & $4.6 \times 10^{-7}$ & IFIT1 & Interferon-induced protein with tetratricopeptide repeats 1 \\
\hline 3.6 & 5.5 & 3.8 & $3.4 \times 10^{-7}$ & $1.0 \times 10^{-4}$ & IFIT3 & Interferon-induced protein with tetratricopeptide repeats 3 \\
\hline 2.5 & 4.4 & 3.9 & $7.6 \times 10^{-7}$ & $2.0 \times 10^{-4}$ & PRDM9 & PR domain containing 9 \\
\hline 4.4 & 6.5 & 4.2 & $2.4 \times 10^{-7}$ & $8.8 \times 10^{-5}$ & RSAD2 & Radical S-adenosyl methionine domain containing 2 \\
\hline 4.9 & 7.0 & 4.3 & $1.5 \times 10^{-8}$ & $1.1 \times 10^{-5}$ & IRF9 & Interferon regulatory factor 9 \\
\hline 5.6 & 7.7 & 4.3 & $4.5 \times 10^{-8}$ & $2.3 \times 10^{-5}$ & IGFBP7 & Insulin like growth factor binding protein 7 \\
\hline 5.2 & 7.4 & 4.4 & $6.1 \times 10^{-6}$ & $1.1 \times 10^{-3}$ & DDX60 & DEAD (Asp-Glu-Ala-Asp) box polypeptide 60 \\
\hline 4.8 & 7.0 & 4.4 & $4.8 \times 10^{-12}$ & $1.5 \times 10^{-8}$ & DEFB1 & Defensin, beta 1 \\
\hline 3.4 & 5.6 & 4.6 & $4.9 \times 10^{-13}$ & $4.7 \times 10^{-9}$ & NEO1 & Neogenin 1 \\
\hline 8.2 & 10.4 & 4.7 & $2.6 \times 10^{-8}$ & $1.5 \times 10^{-5}$ & IFI6 & Interferon, alpha-inducible protein 6 \\
\hline 4.9 & 7.2 & 4.7 & $1.3 \times 10^{-10}$ & $2.3 \times 10^{-7}$ & FXYD4 & FXYD domain containing ion transport regulator 4 \\
\hline 5.1 & 7.3 & 4.9 & $1.6 \times 10^{-7}$ & $6.2 \times 10^{-5}$ & IFI44 & Interferon-induced protein 44 \\
\hline 2.6 & 5.0 & 5.1 & $8.8 \times 10^{-8}$ & $4.1 \times 10^{-5}$ & IFI44L & Interferon-induced protein 44 -like \\
\hline 4.7 & 7.2 & 5.6 & $2.0 \times 10^{-4}$ & $1.3 \times 10^{-2}$ & SPINK1 & Serine peptidase inhibitor, Kazal type 1 \\
\hline 1.9 & 4.4 & 5.8 & $9.8 \times 10^{-8}$ & $4.3 \times 10^{-5}$ & NTS & Neurotensin \\
\hline 4.2 & 6.8 & 6.0 & $2.2 \times 10^{-11}$ & $5.0 \times 10^{-8}$ & OAS2 & 2-5-oligoadenylate synthetase 2 \\
\hline 2.8 & 5.8 & 7.7 & $2.1 \times 10^{-9}$ & $2.2 \times 10^{-6}$ & CFAP126 & Cilia and flagella associated protein 126 \\
\hline
\end{tabular}

Typically, malignant tumor cells respond to high concentrations of glucose or insulin (fed state) by induction of gene transcripts and activation of mTOR signaling to augment glucose transporters, glycolytic enzymes, and enable higher production of succinyl-CoA and malonyl-CoA metabolites, which inhibit fatty acid oxidation as an alternative fuel source, coinciding with the Warburg effect. In this study we show that glycolysis-null KO clones undergo a transcriptomic shift consistent with glucose deprivation signaling (response to long term fasting), with increased transcription of mitochondrial HMGCS2 (HMGCoA synthetase) and PPAR coactivator $-1 \alpha(P G C-1 \alpha)$, both associated with loss of mTOR signaling (24). The high expression of $P G C-1 \alpha$ reported leads to heightened AMPK phospho-signaling, which normally plays a role in long-chain fatty acid catabolism, inactivation of acetyl-CoA carboxylase 
Table II. WT affymetrix human transcriptome: WT versus GPI KO LS174T colon adenocarcinoma cells.

\begin{tabular}{|c|c|c|c|c|c|c|}
\hline $\begin{array}{l}\text { GPI KO } \\
\text { Avg }(\log 2)\end{array}$ & $\begin{array}{c}\text { Ctrl } \\
\text { Avg }(\log 2)\end{array}$ & $\begin{array}{l}\text { Fold } \\
\text { change }\end{array}$ & $p$-Value & $\begin{array}{c}\text { FDR } \\
p \text {-Value }\end{array}$ & $\begin{array}{l}\text { Gene } \\
\text { symbol }\end{array}$ & Description \\
\hline 4.0 & 8.4 & -20.6 & $2.1 \times 10^{-8}$ & $2.0 \times 10^{-5}$ & DKK4 & Dickkopf WNT signaling pathway inhibitor 4 \\
\hline 2.4 & 6.3 & -14.8 & $1.1 \times 10^{-14}$ & $1.7 \times 10^{-10}$ & GJA1 & Gap junction protein alpha 1 \\
\hline 2.8 & 6.3 & -10.7 & $1.3 \times 10^{-5}$ & $2.8 \times 10^{-3}$ & VCAN & Versican \\
\hline 4.5 & 7.6 & -8.4 & $3.0 \times 10^{-4}$ & $2.2 \times 10^{-2}$ & BGN & Biglycan \\
\hline 4.1 & 7.1 & -7.8 & $1.4 \times 10^{-6}$ & $5.0 \times 10^{-4}$ & CALB2 & Calbindin 2 \\
\hline 2.8 & 5.8 & -7.6 & $2.5 \times 10^{-10}$ & $5.5 \times 10^{-7}$ & $\mathrm{AKR} 1 \mathrm{C} 2$ & Aldo-keto reductase family 1 , member $\mathrm{C} 2$ \\
\hline 3.0 & 5.8 & -7.1 & $6.7 \times 10^{-14}$ & $6.5 \times 10^{-10}$ & ZNF607 & Zinc finger protein 607 \\
\hline 2.8 & 5.5 & -6.5 & $4.1 \times 10^{-13}$ & $3.3 \times 10^{-9}$ & SP8 & Sp8 transcription factor \\
\hline 3.1 & 5.7 & -5.9 & $1.0 \times 10^{-11}$ & $5.3 \times 10^{-8}$ & DSC3 & Desmocollin 3 \\
\hline 7.3 & 9.8 & -5.8 & $8.1 \times 10^{-15}$ & $1.7 \times 10^{-10}$ & GPI & Glucose 6-phosphate isomerase \\
\hline 3.7 & 6.2 & -5.7 & $1.7 \times 10^{-10}$ & $4.3 \times 10^{-7}$ & APCDD1 & Adenomatosis polyposis coli down-regulated 1 \\
\hline 5.0 & 7.3 & -4.7 & $5.0 \times 10^{-11}$ & $1.7 \times 10^{-7}$ & HOXB9 & Homeobox B9 \\
\hline 4.1 & 6.3 & -4.7 & $6.5 \times 10^{-8}$ & $5.2 \times 10^{-5}$ & TIMP1 & TIMP metallopeptidase inhibitor 1 \\
\hline 2.9 & 5.2 & -4.7 & $4.5 \times 10^{-8}$ & $4.0 \times 10^{-5}$ & PCDHB13 & Protocadherin beta 13 \\
\hline 5.1 & 7.3 & -4.7 & $1.1 \times 10^{-11}$ & $5.3 \times 10^{-8}$ & CCND2 & Cyclin D2 \\
\hline 6.0 & 8.2 & -4.5 & $1.8 \times 10^{-6}$ & $6.0 \times 10^{-4}$ & GPX2 & Glutathione peroxidase 2 \\
\hline 4.5 & 6.6 & -4.4 & $1.4 \times 10^{-12}$ & $9.5 \times 10^{-9}$ & HOXB6; HOXB3 & Homeobox B6; homeobox B3 \\
\hline 2.8 & 4.9 & -4.2 & $6.3 \times 10^{-7}$ & $3.0 \times 10^{-4}$ & HUNK & Hormonally up-regulated Neu-associated kinase \\
\hline 3.2 & 5.2 & -3.9 & $5.6 \times 10^{-6}$ & $1.5 \times 10^{-3}$ & HULC & $\begin{array}{c}\text { Hepatocellular carcinoma up-regulated } \\
\text { long non-coding RNA }\end{array}$ \\
\hline 3.9 & 5.8 & -3.9 & $1.3 \times 10^{-5}$ & $2.9 \times 10^{-3}$ & EIF2B5-AS1 & EIF2B5 antisense RNA 1 \\
\hline 3.9 & 5.7 & -3.7 & $6.4 \times 10^{-10}$ & $1.3 \times 10^{-6}$ & HOXB5 & Homeobox B5 \\
\hline 6.4 & 8.3 & -3.6 & $7.4 \times 10^{-6}$ & $1.8 \times 10^{-3}$ & ALDH3A1 & Aldehyde dehydrogenase 3 family, member A1 \\
\hline 5.8 & 7.6 & -3.4 & $5.0 \times 10^{-4}$ & $2.8 \times 10^{-2}$ & ANXA1 & Annexin A1 \\
\hline 5.8 & 7.6 & -3.4 & $1.3 \times 10^{-10}$ & $3.7 \times 10^{-7}$ & AKR1B10 & $\begin{array}{l}\text { Aldo-keto reductase family } 1 \text {, } \\
\text { member B10 (aldose reductase) }\end{array}$ \\
\hline 2.8 & 4.5 & -3.3 & $6.2 \times 10^{-6}$ & $1.6 \times 10^{-3}$ & MIR196A1 & MicroRNA 196a-1 \\
\hline 3.6 & 5.3 & -3.2 & $5.0 \times 10^{-6}$ & $1.4 \times 10^{-3}$ & HBE1 & Hemoglobin, epsilon 1 \\
\hline 4.8 & 6.5 & -3.2 & $5.1 \times 10^{-6}$ & $1.4 \times 10^{-3}$ & KLK7 & Kallikrein related peptidase 7 \\
\hline 4.1 & 5.7 & -3.1 & $2.0 \times 10^{-4}$ & $1.5 \times 10^{-2}$ & CRYBB1 & Crystallin beta B1 \\
\hline 3.7 & 5.3 & -3.1 & $6.2 \times 10^{-8}$ & $5.1 \times 10^{-5}$ & SKAP1 & Src kinase associated phosphoprotein 1 \\
\hline 2.5 & 4.1 & -3.1 & $5.0 \times 10^{-4}$ & $3.0 \times 10^{-2}$ & OR51B4 & Olfactory receptor, family 51 , subfamily $\mathrm{B}$, member 4 \\
\hline 1.8 & 3.4 & -3.1 & $4.0 \times 10^{-4}$ & $2.6 \times 10^{-2}$ & MIR548X & MicroRNA 548x \\
\hline 3.1 & 4.7 & -3.0 & $2.0 \times 10^{-4}$ & $1.8 \times 10^{-2}$ & TM4SF4 & Transmembrane $4 \mathrm{~L}$ six family member 4 \\
\hline 4.4 & 5.9 & -3.0 & $3.3 \times 10^{-5}$ & $5.4 \times 10^{-3}$ & ANKRD22 & Ankyrin repeat domain 22 \\
\hline 4.2 & 5.7 & -2.9 & $2.3 \times 10^{-9}$ & $3.8 \times 10^{-6}$ & MFSD2A & Major facilitator superfamily domain containing $2 \mathrm{~A}$ \\
\hline 4.2 & 5.7 & -2.9 & $1.3 \times 10^{-7}$ & $8.8 \times 10^{-5}$ & THSD4 & Thrombospondin type 1 domain containing 4 \\
\hline 2.7 & 4.2 & -2.8 & $2.0 \times 10^{-8}$ & $2.0 \times 10^{-5}$ & ALDH1A1 & Aldehyde dehydrogenase 1 family, member A1 \\
\hline 6.0 & 7.5 & -2.8 & $8.8 \times 10^{-10}$ & $1.6 \times 10^{-6}$ & PLA2G4A & $\begin{array}{l}\text { Phospholipase A2, group IVA } \\
\text { (cytosolic, calcium-dependent) }\end{array}$ \\
\hline 4.4 & 5.8 & -2.7 & $1.5 \times 10^{-9}$ & $2.5 \times 10^{-6}$ & FAM171B & Family with sequence similarity 171 , member B \\
\hline 8.0 & 9.3 & -2.6 & $1.1 \times 10^{-5}$ & $2.4 \times 10^{-3}$ & DPEP1 & Dipeptidase 1 (renal) \\
\hline 6.1 & 7.5 & -2.6 & $1.5 \times 10^{-8}$ & $1.6 \times 10^{-5}$ & ID1 & $\begin{array}{l}\text { Inhibitor of DNA binding 1, dominant } \\
\text { negative helix-loop-helix protein }\end{array}$ \\
\hline 3.3 & 4.7 & -2.6 & $2.1 \times 10^{-8}$ & $2.0 \times 10^{-5}$ & ANOS1 & Anosmin 1 \\
\hline 3.9 & 5.3 & -2.6 & $2.3 \times 10^{-5}$ & $4.2 \times 10^{-3}$ & FAR2P1 & Fatty acyl-CoA reductase 2 pseudogene 1 \\
\hline 4.7 & 6.1 & -2.6 & $6.8 \times 10^{-6}$ & $1.7 \times 10^{-3}$ & SLC6A20 & $\begin{array}{l}\text { Solute carrier family } 6 \\
\text { (proline IMINO transporter), member } 20\end{array}$ \\
\hline 1.6 & 2.9 & -2.5 & $3.1 \times 10^{-6}$ & $1.0 \times 10^{-3}$ & GPX8 & Glutathione peroxidase 8 (putative) \\
\hline 3.7 & 5.0 & -2.5 & $6.6 \times 10^{-7}$ & $3.0 \times 10^{-4}$ & $\mathrm{AKR} 1 \mathrm{C} 1$ & Aldo-keto reductase family 1 , member $\mathrm{C} 1$ \\
\hline 2.7 & 4.0 & -2.5 & $2.0 \times 10^{-7}$ & $1.0 \times 10^{-4}$ & MECOM & MDS1 and EVI1 complex locus \\
\hline 4.6 & 5.9 & -2.5 & $3.0 \times 10^{-9}$ & $4.5 \times 10^{-6}$ & PLA2G16 & Phospholipase A2, group XVI \\
\hline 5.3 & 6.6 & -2.5 & $8.4 \times 10^{-6}$ & $2.0 \times 10^{-3}$ & AREG & Amphiregulin \\
\hline 5.1 & 6.4 & -2.5 & $1.8 \times 10^{-6}$ & $6.0 \times 10^{-4}$ & AKR1C3 & Aldo-keto reductase family 1 , member $\mathrm{C} 3$ \\
\hline 3.0 & 4.3 & -2.4 & $7.6 \times 10^{-8}$ & $5.9 \times 10^{-} 5$ & CCND2-AS1 & CCND2 antisense RNA 1 \\
\hline 2.3 & 3.6 & -2.4 & $2.4 \times 10^{-3}$ & $7.2 \times 10^{-2}$ & ANKRD36BP1 & Ankyrin repeat domain $36 \mathrm{~B}$ pseudogene 1 \\
\hline 5.8 & 7.0 & -2.4 & $1.3 \times 10^{-5}$ & $2.8 \times 10^{-3}$ & AREG & Amphiregulin \\
\hline 9.0 & 10.3 & -2.4 & $1.0 \times 10^{-4}$ & $1.4 \times 10^{-2}$ & ND6 & NADH dehydrogenase, subunit 6 (complex I) \\
\hline
\end{tabular}


Table II. Continued

\begin{tabular}{|c|c|c|c|c|c|c|}
\hline $\begin{array}{l}\text { GPI KO } \\
\text { Avg }(\log 2)\end{array}$ & $\begin{array}{c}\text { Ctrl } \\
\text { Avg }(\log 2)\end{array}$ & $\begin{array}{l}\text { Fold } \\
\text { change }\end{array}$ & $p$-Value & $\begin{array}{c}\text { FDR } \\
p \text {-Value }\end{array}$ & $\begin{array}{l}\text { Gene } \\
\text { symbol }\end{array}$ & Description \\
\hline 1.7 & 2.9 & -2.4 & $1.0 \times 10^{-4}$ & $1.4 \times 10^{-2}$ & GATSL2 & GATS protein-like 2 \\
\hline 3.3 & 4.5 & -2.4 & $4.1 \times 10^{-5}$ & $6.2 \times 10^{-3}$ & IGFL4 & IGF like family member 4 \\
\hline 2.9 & 4.1 & -2.3 & $9.2 \times 10^{-8}$ & $6.8 \times 10^{-5}$ & HOXB3 & Homeobox B3 \\
\hline 5.8 & 7.0 & -2.3 & $3.0 \times 10^{-5}$ & $5.0 \times 10^{-3}$ & ADIRF & Adipogenesis regulatory factor \\
\hline 6.2 & 7.4 & -2.3 & $8.2 \times 10^{-11}$ & $2.5 \times 10^{-7}$ & SEC31A & SEC31 homolog A, COPII coat complex component \\
\hline 5.0 & 6.2 & -2.3 & $1.7 \times 10^{-8}$ & $1.7 \times 10^{-5}$ & HOXB7 & Homeobox B7 \\
\hline 2.5 & 3.7 & -2.3 & $8.2 \times 10^{-7}$ & $4.0 \times 10^{-4}$ & BCHE & Butyrylcholinesterase \\
\hline 7.1 & 8.3 & -2.3 & $1.3 \times 10^{-2}$ & $1.7 \times 10^{-1}$ & MUC6 & Mucin 6, oligomeric mucus/gel-forming \\
\hline 3.9 & 5.1 & -2.3 & $2.5 \times 10^{-6}$ & $8.0 \times 10^{-4}$ & BCL11A & B-cell CLL/lymphoma 11A (zinc finger protein) \\
\hline 5.7 & 6.9 & -2.3 & $3.1 \times 10^{-2}$ & $2.6 \times 10^{-1}$ & ROCK1P1 & $\begin{array}{l}\text { Rho-associated, coiled-coil containing } \\
\text { protein kinase } 1 \text { pseudogene } 1\end{array}$ \\
\hline 9.1 & 10.3 & -2.3 & $7.5 \times 10^{-5}$ & $9.2 \times 10^{-3}$ & SLC7A11 & $\begin{array}{l}\text { Solute carrier family } 7 \text { (anionic amino } \\
\text { acid transporter light chain, xc- system), member } 11\end{array}$ \\
\hline 3.4 & 4.5 & -2.2 & $1.9 \times 10^{-8}$ & $1.9 \times 10^{-5}$ & SATB1 & SATB homeobox 1 \\
\hline 4.9 & 6.1 & -2.2 & $1.2 \times 10^{-7}$ & $8.2 \times 10^{-5}$ & IFITM2 & Interferon induced transmembrane protein 2 \\
\hline 4.4 & 5.6 & -2.2 & $1.1 \times 10^{-6}$ & $5.0 \times 10^{-4}$ & SIPA1L2 & Signal-induced proliferation-associated 1 like 2 \\
\hline 2.2 & 3.4 & -2.2 & $2.7 \times 10^{-6}$ & $9.0 \times 10^{-4}$ & GXYLT2 & Glucoside xylosyltransferase 2 \\
\hline 4.8 & 5.9 & -2.2 & $1.7 \times 10^{-3}$ & $6.1 \times 10^{-2}$ & UCA1 & Urothelial cancer associated 1 (non-protein coding) \\
\hline 8.1 & 9.2 & -2.2 & $1.4 \times 10^{-2}$ & $1.8 \times 10^{-1}$ & CTSE & Cathepsin E \\
\hline 3.5 & 4.6 & -2.2 & $2.8 \times 10^{-5}$ & $4.7 \times 10^{-3}$ & $\mathrm{ZC} 3 \mathrm{H} 12 \mathrm{C}$ & Zinc finger $\mathrm{CCCH}$-type containing $12 \mathrm{C}$ \\
\hline 5.4 & 6.5 & -2.2 & $3.0 \times 10^{-8}$ & $2.8 \times 10^{-5}$ & ABCC3 & ATP binding cassette subfamily $\mathrm{C}$ member 3 \\
\hline 2.7 & 3.8 & -2.2 & $4.7 \times 10^{-3}$ & $1.0 \times 10^{-1}$ & CEP152 & Centrosomal protein $152 \mathrm{kDa}$ \\
\hline 2.3 & 3.4 & -2.2 & $4.0 \times 10^{-4}$ & $2.6 \times 10^{-2}$ & LRRC7 & Leucine rich repeat containing 7 \\
\hline 1.9 & 3.0 & -2.2 & $2.4 \times 10^{-3}$ & $7.2 \times 10^{-2}$ & FAM35DP & $\begin{array}{l}\text { Family with sequence similarity } 35, \\
\text { member A pseudogene }\end{array}$ \\
\hline 5.8 & 6.9 & -2.2 & $7.0 \times 10^{-5}$ & $9.0 \times 10^{-3}$ & LIPH & Lipase, member $\mathrm{H}$ \\
\hline 3.4 & 4.6 & -2.2 & $9.0 \times 10^{-4}$ & $4.1 \times 10^{-2}$ & DIP2A-IT1 & DIP2A intronic transcript 1 \\
\hline 3.4 & 4.6 & -2.2 & $1.8 \times 10^{-2}$ & $2.0 \times 10^{-1}$ & DPY19L2P1 & DPY19L2 pseudogene 1 \\
\hline 2.4 & 3.5 & -2.1 & $1.9 \times 10^{-2}$ & $2.1 \times 10^{-1}$ & PIRC66 & Piwi-interacting RNA cluster 66 \\
\hline 3.8 & 4.9 & -2.1 & $1.8 \times 10^{-2}$ & $2.0 \times 10^{-1}$ & $\begin{array}{l}\text { HIST1H2AM; } \\
\text { HIST1H3J }\end{array}$ & Histone cluster 1, H2am; histone cluster $1, \mathrm{H} 3 \mathrm{j}$ \\
\hline 3.0 & 4.0 & -2.1 & $3.0 \times 10^{-5}$ & $5.0 \times 10^{-3}$ & PAQR8 & Progestin and adipoQ receptor family member VIII \\
\hline 5.2 & 6.3 & -2.1 & $3.0 \times 10^{-4}$ & $2.3 \times 10^{-2}$ & TM4SF1 & Transmembrane $4 \mathrm{~L}$ six family member 1 \\
\hline 4.9 & 6.0 & -2.1 & $1.8 \times 10^{-2}$ & $2.0 \times 10^{-1}$ & GRIN2B & Glutamate receptor, ionotropic, $\mathrm{N}$-methyl $\mathrm{D}$-aspartate $2 \mathrm{~B}$ \\
\hline 5.0 & 6.0 & -2.1 & $3.2 \times 10^{-6}$ & $1.0 \times 10^{-3}$ & CD44 & CD44 molecule (Indian blood group) \\
\hline 4.9 & 5.9 & -2.1 & $3.0 \times 10^{-3}$ & $8.0 \times 10^{-2}$ & CORO1A & Coronin, actin binding protein, $1 \mathrm{~A}$ \\
\hline 3.6 & 4.7 & -2.1 & $2.0 \times 10^{-4}$ & $1.9 \times 10^{-2}$ & ANKRD20A5P & $\begin{array}{l}\text { Ankyrin repeat domain } 20 \\
\text { family, member A5, pseudogene }\end{array}$ \\
\hline 5.1 & 6.1 & -2.1 & $2.2 \times 10^{-5}$ & $4.0 \times 10^{-3}$ & FGFBP1 & Fibroblast growth factor binding protein 1 \\
\hline 2.4 & 3.5 & -2.1 & $2.9 \times 10^{-2}$ & $2.5 \times 10^{-1}$ & MIR4497 & MicroRNA 4497 \\
\hline 2.2 & 3.3 & -2.1 & $2.7 \times 10^{-5}$ & $4.7 \times 10^{-3}$ & RAB29 & RAB29, member RAS oncogene family \\
\hline 5.2 & 6.3 & -2.1 & $4.6 \times 10^{-7}$ & $3.0 \times 10^{-4}$ & SERPINB5 & $\begin{array}{l}\text { Serpin peptidase inhibitor, clade B } \\
\text { (ovalbumin), member } 5\end{array}$ \\
\hline 2.3 & 3.3 & -2.1 & $2.4 \times 10^{-2}$ & $2.3 \times 10^{-1}$ & $\mathrm{CCDC} 30$ & Coiled-coil domain containing 30 \\
\hline 4.5 & 5.6 & -2.1 & $1.1 \times 10^{-2}$ & $1.6 \times 10^{-1}$ & $\begin{array}{l}\text { MUC5AC; } \\
\text { MUC5B }\end{array}$ & $\begin{array}{l}\text { Mucin 5AC, oligomeric mucus/gel-forming; } \\
\text { mucin 5B, oligomeric mucus/gel-forming }\end{array}$ \\
\hline 6.8 & 7.8 & -2.0 & $1.2 \times 10^{-5}$ & $2.7 \times 10^{-3}$ & DCBLD2 & Discoidin, CUB and LCCL domain containing 2 \\
\hline 3.1 & 4.2 & -2.0 & $5.1 \times 10^{-6}$ & $1.4 \times 10^{-3}$ & LYZ & Lysozyme \\
\hline 4.1 & 5.1 & -2.0 & $6.5 \times 10^{-7}$ & $3.0 \times 10^{-4}$ & CD33 & CD33 molecule \\
\hline 3.8 & 4.8 & -2.0 & $8.0 \times 10^{-5}$ & $9.7 \times 10^{-3}$ & IFIT1 & Interferon-induced protein with tetratricopeptide repeats 1 \\
\hline 1.8 & 2.8 & -2.0 & $1.0 \times 10^{-3}$ & $4.6 \times 10^{-2}$ & SPDYE3 & Speedy/RINGO cell cycle regulator family member E3 \\
\hline 5.1 & 6.1 & -2.0 & $1.8 \times 10^{-6}$ & $6.0 \times 10^{-4}$ & TFCP2 & Transcription factor $\mathrm{CP} 2$ \\
\hline 8.1 & 9.1 & -2.0 & $1.7 \times 10^{-3}$ & $5.9 \times 10^{-2}$ & KLK6 & Kallikrein related peptidase 6 \\
\hline 2.2 & 3.2 & -2.0 & $4.0 \times 10^{-3}$ & $9.4 \times 10^{-2}$ & MIR626 & MicroRNA 626 \\
\hline 4.8 & 5.8 & -2.0 & $3.0 \times 10^{-4}$ & $2.4 \times 10^{-2}$ & CES1; CES1P1 & 1 Carboxylesterase 1 ; carboxylesterase 1 pseudogene 1 \\
\hline 2.6 & 3.6 & -2.0 & $4.5 \times 10^{-2}$ & $3.1 \times 10^{-1}$ & MIR548X2 & MicroRNA 548x-2 \\
\hline 4.7 & 3.7 & 2.0 & $6.6 \times 10^{-5}$ & $8.6 \times 10^{-3}$ & MIR210HG & MIR210 host gene \\
\hline 9.1 & 8.1 & 2.0 & $2.4 \times 10^{-6}$ & $8.0 \times 10^{-4}$ & TXNIP & Thioredoxin interacting protein \\
\hline
\end{tabular}

Table II. Continued 
Table II. Continued

\begin{tabular}{|c|c|c|c|c|c|c|}
\hline $\begin{array}{l}\text { GPI KO } \\
\text { Avg }(\log 2)\end{array}$ & $\begin{array}{c}\text { Ctrl } \\
\text { Avg }(\log 2)\end{array}$ & $\begin{array}{l}\text { Fold } \\
\text { change }\end{array}$ & $p$-Value & $\begin{array}{c}\text { FDR } \\
p \text {-Value }\end{array}$ & $\begin{array}{l}\text { Gene } \\
\text { symbol }\end{array}$ & Description \\
\hline 3.7 & 2.7 & 2.0 & $2.6 \times 10^{-2}$ & $2.4 \times 10^{-1}$ & MIR4731 & MicroRNA 4731 \\
\hline 6.1 & 5.0 & 2.1 & $1.4 \times 10^{-5}$ & $3.0 \times 10^{-3}$ & RILP & Rab interacting lysosomal protein \\
\hline 4.3 & 3.3 & 2.1 & $1.2 \times 10^{-6}$ & $5.0 \times 10^{-4}$ & ZSCAN12P1 & $\begin{array}{l}\text { Zinc finger and SCAN domain } \\
\text { containing } 12 \text { pseudogene } 1\end{array}$ \\
\hline 5.1 & 4.0 & 2.1 & $1.7 \times 10^{-3}$ & $6.1 \times 10^{-2}$ & IL2RG & Interleukin 2 receptor, gamma \\
\hline 4.4 & 3.4 & 2.1 & $6.1 \times 10^{-5}$ & $8.1 \times 10^{-3}$ & LOXL1 & Lysyl oxidasx10-like 1 \\
\hline 6.1 & 5.0 & 2.1 & $7.6 \times 10^{-7}$ & $4.0 \times 10^{-4}$ & PIP5K1B & Phosphatidylinositol-4-phosphate 5-kinase, type I, beta \\
\hline 4.5 & 3.5 & 2.1 & $3.3 \times 10^{-3}$ & $8.5 \times 10^{-2}$ & SULT1A1 & Sulfotransferase family $1 \mathrm{~A}$ member 1 \\
\hline 7.7 & 6.6 & 2.1 & $1.5 \times 10^{-5}$ & $3.2 \times 10^{-3}$ & ZG16B & Zymogen granule protein 16B \\
\hline 8.5 & 7.5 & 2.1 & $4.3 \times 10^{-6}$ & $1.2 \times 10^{-3}$ & $\mathrm{CDH} 17$ & Cadherin 17, LI cadherin (liver-intestine) \\
\hline 3.2 & 2.1 & 2.1 & $7.8 \times 10^{-6}$ & $1.9 \times 10^{-3}$ & SULT1C2P1 & Sulfotransferase family $1 \mathrm{C}$ member 2 pseudogene 1 \\
\hline 6.3 & 5.3 & 2.1 & $8.5 \times 10^{-5}$ & $1.0 \times 10^{-2}$ & ZBTB38 & Zinc finger and BTB domain containing 38 \\
\hline 5.4 & 4.3 & 2.1 & $3.2 \times 10^{-7}$ & $2.0 \times 10^{-4}$ & ADM & Adrenomedullin \\
\hline 3.8 & 2.8 & 2.1 & $3.1 \times 10^{-5}$ & $5.1 \times 10^{-3}$ & REG4 & Regenerating islet-derived family, member 4 \\
\hline 7.3 & 6.2 & 2.1 & $4.2 \times 10^{-3}$ & $9.6 \times 10^{-2}$ & VEGFA & Vascular endothelial growth factor A \\
\hline 5.2 & 4.1 & 2.1 & $6.9 \times 10^{-5}$ & $8.9 \times 10^{-3}$ & $\mathrm{ENO} 2$ & Enolase 2 (gamma, neuronal) \\
\hline 7.5 & 6.4 & 2.1 & $1.5 \times 10^{-5}$ & $3.2 \times 10^{-3}$ & HK2 & Hexokinase 2 \\
\hline 7.3 & 6.2 & 2.1 & $3.4 \times 10^{-6}$ & $1.0 \times 10^{-3}$ & ARG2 & Arginase 2 \\
\hline 6.7 & 5.6 & 2.1 & $4.7 \times 10^{-6}$ & $1.3 \times 10^{-3}$ & TMEM139 & Transmembrane protein 139 \\
\hline 3.0 & 1.9 & 2.1 & $7.0 \times 10^{-4}$ & $3.5 \times 10^{-2}$ & MIR3188 & MicroRNA 3188 \\
\hline 6.2 & 5.1 & 2.1 & $2.9 \times 10^{-6}$ & $9.0 \times 10^{-4}$ & PTPRB & Protein tyrosine phosphatase, receptor type, B \\
\hline 5.1 & 4.0 & 2.2 & $1.8 \times 10^{-3}$ & $6.1 \times 10^{-2}$ & C1orf233 & Chromosome 1 open reading frame 233 \\
\hline 7.0 & 5.9 & 2.2 & $6.0 \times 10^{-4}$ & $3.2 \times 10^{-2}$ & RIT1 & Ras-like without CAAX 1 \\
\hline 6.2 & 5.1 & 2.2 & $1.6 \times 10^{-6}$ & $6.0 \times 10^{-4}$ & TMEM141 & Transmembrane protein 141 \\
\hline 5.6 & 4.5 & 2.2 & $2.5 \times 10^{-5}$ & $4.4 \times 10^{-3}$ & LOC105371592 & Atherin-like \\
\hline 4.7 & 3.6 & 2.2 & $2.3 \times 10^{-3}$ & $7.0 \times 10^{-2}$ & CPEB3 & Cytoplasmic polyadenylation element binding protein 3 \\
\hline 3.7 & 2.6 & 2.2 & $2.7 \times 10^{-3}$ & $7.6 \times 10^{-2}$ & NBPF4 & Neuroblastoma breakpoint family, member 4 \\
\hline 3.2 & 2.1 & 2.2 & $2.2 \times 10^{-7}$ & $1.0 \times 10^{-4}$ & ANKRD45 & Ankyrin repeat domain 45 \\
\hline 7.9 & 6.8 & 2.2 & $1.2 \times 10^{-7}$ & $8.2 \times 10^{-5}$ & ITPK1 & Inositol-tetrakisphosphate 1-kinase \\
\hline 3.7 & 2.5 & 2.2 & $6.8 \times 10^{-8}$ & $5.4 \times 10^{-5}$ & CTSS & Cathepsin S \\
\hline 6.0 & 4.9 & 2.2 & $1.6 \times 10^{-5}$ & $3.2 \times 10^{-3}$ & DHRS3 & Dehydrogenase/reductase (SDR family) member 3 \\
\hline 6.0 & 4.9 & 2.2 & $9.9 \times 10^{-3}$ & $1.5 \times 10^{-} 1$ & IRF9 & Interferon regulatory factor 9 \\
\hline 5.1 & 3.9 & 2.2 & $3.0 \times 10^{-4}$ & $2.4 \times 10^{-} 2$ & NDUFA4L2 & $\begin{array}{l}\text { NADH dehydrogenase (ubiquinone) } \\
1 \text { alpha subcomplex, 4-like } 2\end{array}$ \\
\hline 5.6 & 4.5 & 2.2 & $4.9 \times 10^{-7}$ & $3.0 \times 10^{-4}$ & KLK1 & Kallikrein 1 \\
\hline 5.8 & 4.6 & 2.2 & $2.0 \times 10^{-4}$ & $2.0 \times 10^{-2}$ & $\begin{array}{l}\text { RASA4B; } \\
\text { RASA4 }\end{array}$ & $\begin{array}{l}\text { RAS p } 21 \text { protein activator } 4 \mathrm{~B} \\
\text { RAS p21 protein activator } 4\end{array}$ \\
\hline 5.3 & 4.2 & 2.2 & $1.4 \times 10^{-5}$ & $3.0 \times 10^{-3}$ & SMIM24 & Small integral membrane protein 24 \\
\hline 5.1 & 3.9 & 2.2 & $1.0 \times 10^{-4}$ & $1.2 \times 10^{-2}$ & SMAD5 & SMAD family member 5 \\
\hline 5.8 & 4.6 & 2.2 & $1.7 \times 10^{-2}$ & $1.9 \times 10^{-1}$ & АBCC6P2 & $\begin{array}{l}\text { ATP binding cassette subfamily } \mathrm{C} \\
\text { member } 6 \text { pseudogene } 2\end{array}$ \\
\hline 6.5 & 5.4 & 2.2 & $4.5 \times 10^{-3}$ & $1.0 \times 10^{-1}$ & LINC01207 & Long intergenic non-protein coding RNA 1207 \\
\hline 7.7 & 6.5 & 2.2 & $3.4 \times 10^{-6}$ & $1.0 \times 10^{-3}$ & $\mathrm{ABCB} 1$ & ATP binding cassette subfamily B member 1 \\
\hline 8.4 & 7.2 & 2.2 & $1.2 \times 10^{-6}$ & $5.0 \times 10^{-4}$ & PLOD1 & Procollagen-lysine, 2-oxoglutarate 5-dioxygenase 1 \\
\hline 7.9 & 6.7 & 2.3 & $3.9 \times 10^{-6}$ & $1.1 \times 10^{-3}$ & KRT19 & Keratin 19 , type I \\
\hline 6.9 & 5.7 & 2.3 & $3.0 \times 10^{-4}$ & $2.3 \times 10^{-2}$ & SECTM1 & Secreted and transmembrane 1 \\
\hline 4.5 & 3.3 & 2.3 & $2.0 \times 10^{-4}$ & $1.6 \times 10^{-2}$ & DMGDH & Dimethylglycine dehydrogenase \\
\hline 4.2 & 3.1 & 2.3 & $1.0 \times 10^{-4}$ & $1.3 \times 10^{-2}$ & EGLN3 & Egl-9 family hypoxia-inducible factor 3 \\
\hline 5.1 & 3.9 & 2.3 & $2.8 \times 10^{-5}$ & $4.9 \times 10^{-3}$ & NXPH4 & Neurexophilin 4 \\
\hline 9.7 & 8.5 & 2.3 & $3.0 \times 10^{-4}$ & $2.1 \times 10^{-2}$ & NRN1 & Neuritin 1 \\
\hline 6.9 & 5.7 & 2.3 & $7.3 \times 10^{-10}$ & $1.4 \times 10^{-6}$ & FAM84A & Family with sequence similarity 84 , member A \\
\hline 8.8 & 7.6 & 2.3 & $9.7 \times 10^{-5}$ & $1.1 \times 10^{-2}$ & PDK1 & Pyruvate dehydrogenase kinase, isozyme 1 \\
\hline 5.5 & 4.2 & 2.3 & $2.5 \times 10^{-5}$ & $4.4 \times 10^{-3}$ & SLC6A10P & $\begin{array}{l}\text { Solute carrier family } 6 \\
\text { (neurotransmitter transporter), member 10, pseudogene }\end{array}$ \\
\hline 7.5 & 6.3 & 2.4 & $4.6 \times 10^{-3}$ & $1.0 \times 10^{-1}$ & INSIG2 & Insulin induced gene 2 \\
\hline 2.5 & 1.3 & 2.4 & $6.0 \times 10^{-4}$ & $3.1 \times 10^{-2}$ & $\begin{array}{l}\text { MIR4678; } \\
\text { MINPP1 }\end{array}$ & $\begin{array}{l}\text { MicroRNA 4678; multiple } \\
\text { inositol-polyphosphate phosphatase } 1\end{array}$ \\
\hline 7.1 & 5.8 & 2.4 & $1.7 \times 10^{-8}$ & $1.7 \times 10^{-5}$ & IL33 & Interleukin 33 \\
\hline
\end{tabular}

Table II. Continued 
Table II. Continued

\begin{tabular}{|c|c|c|c|c|c|c|}
\hline $\begin{array}{l}\text { GPI KO } \\
\text { Avg }(\log 2)\end{array}$ & $\begin{array}{c}\text { Ctrl } \\
\text { Avg }(\log 2)\end{array}$ & $\begin{array}{l}\text { Fold } \\
\text { change }\end{array}$ & $p$-Value & $\begin{array}{c}\text { FDR } \\
p \text {-Value }\end{array}$ & $\begin{array}{l}\text { Gene } \\
\text { symbol }\end{array}$ & Description \\
\hline 3.4 & 2.2 & 2.4 & $7.2 \times 10^{-3}$ & $1.3 \times 10^{-1}$ & VTCN1 & V-set domain containing $\mathrm{T}$ cell activation inhibitor 1 \\
\hline 10.5 & 9.2 & 2.4 & $4.2 \times 10^{-5}$ & $6.4 \times 10^{-3}$ & CYP2S1 & Cytochrome P450, family 2 , subfamily $\mathrm{S}$, polypeptide 1 \\
\hline 4.4 & 3.1 & 2.4 & $3.0 \times 10^{-4}$ & $2.2 \times 10^{-2}$ & KRTAP5-5 & Keratin associated protein $5-5$ \\
\hline 3.5 & 2.2 & 2.4 & $1.1 \times 10^{-2}$ & $1.6 \times 10^{-1}$ & IFNA5 & Interferon, alpha 5 \\
\hline 6.8 & 5.5 & 2.4 & $6.2 \times 10^{-11}$ & $2.0 \times 10^{-7}$ & SCIN & Scinderin \\
\hline 7.6 & 6.3 & 2.4 & $2.1 \times 10^{-6}$ & $7.0 \times 10^{-4}$ & $\mathrm{ACY} 3$ & Aminoacylase 3 \\
\hline 7.8 & 6.5 & 2.4 & $6.6 \times 10^{-7}$ & $3.0 \times 10^{-4}$ & FRRS1 & Ferric-chelate reductase 1 \\
\hline 6.4 & 5.1 & 2.4 & $4.6 \times 10^{-8}$ & $4.0 \times 10^{-5}$ & EFNA1 & Ephrin-A1 \\
\hline 4.2 & 2.9 & 2.5 & $3.7 \times 10^{-3}$ & $9.0 \times 10^{-2}$ & C6orf222 & Chromosome 6 open reading frame 222 \\
\hline 7.2 & 5.9 & 2.5 & $1.5 \times 10^{-6}$ & $6.0 \times 10^{-4}$ & FXYD3 & FXYD domain containing ion transport regulator 3 \\
\hline 5.0 & 3.7 & 2.5 & $1.5 \times 10^{-5}$ & $3.1 \times 10^{-3}$ & KCNQ2 & $\begin{array}{l}\text { Potassium channel, voltage gated } \\
\text { KQT-like subfamily Q, member } 2\end{array}$ \\
\hline 3.0 & 1.7 & 2.5 & $1.5 \times 10^{-2}$ & $1.9 \times 10^{-1}$ & $\begin{array}{l}\text { UGT2B } 17 \\
\text { UGT2B } 15\end{array}$ & $\begin{array}{l}\text { UDP glucuronosyltransferase } 2 \text { family, } \\
\text { polypeptide B17; UDP glucuronosyltransferase } \\
2 \text { family, polypeptide B15 }\end{array}$ \\
\hline 8.0 & 6.7 & 2.5 & $7.5 \times 10^{-6}$ & $1.9 \times 10^{-3}$ & CD68 & CD68 molecule \\
\hline 4.5 & 3.2 & 2.5 & $3.0 \times 10^{-2}$ & $2.5 \times 10^{-1}$ & RIMBP3C & RIMS binding protein $3 \mathrm{C}$ \\
\hline 8.6 & 7.3 & 2.6 & $6.1 \times 10^{-5}$ & $8.2 \times 10^{-3}$ & TDP2 & Tyrosyl-DNA phosphodiesterase 2 \\
\hline 3.1 & 1.8 & 2.6 & $2.2 \times 10^{-3}$ & $6.9 \times 10^{-2}$ & MIR519A1 & MicroRNA 519a-1 \\
\hline 7.3 & 5.9 & 2.6 & $6.0 \times 10^{-8}$ & $5.0 \times 10^{-5}$ & MRGPRE & MAS-related GPR, member E \\
\hline 3.9 & 2.5 & 2.6 & $6.1 \times 10^{-6}$ & $1.6 \times 10^{-3}$ & CATSPERB & Catsper channel auxiliary subunit beta \\
\hline 6.6 & 5.2 & 2.6 & $3.9 \times 10^{-3}$ & $9.2 \times 10^{-2}$ & CA9 & Carbonic anhydrase IX \\
\hline 5.8 & 4.4 & 2.6 & $1.4 \times 10^{-8}$ & $1.5 \times 10^{-5}$ & MAGEA3 & MAGE family member A3 \\
\hline 8.2 & 6.8 & 2.6 & $3.0 \times 10^{-4}$ & $2.1 \times 10^{-2}$ & NEAT1 & $\begin{array}{l}\text { Nuclear paraspeckle assembly transcript } 1 \\
\text { (non-protein coding) }\end{array}$ \\
\hline 7.2 & 5.8 & 2.6 & $4.5 \times 10^{-8}$ & $4.0 \times 10^{-5}$ & TINAGL1 & Tubulointerstitial nephritis antigen-like 1 \\
\hline 6.5 & 5.1 & 2.7 & $4.8 \times 10^{-5}$ & $7.1 \times 10^{-3}$ & BHLHE40 & Basic helix-loop-helix family, member e40 \\
\hline 6.0 & 4.6 & 2.7 & $3.0 \times 10^{-4}$ & $2.3 \times 10^{-2}$ & MT1F & Metallothionein $1 \mathrm{~F}$ \\
\hline 5.0 & 3.6 & 2.7 & $4.0 \times 10^{-7}$ & $2.0 \times 10^{-4}$ & FBXO17 & F-box protein 17 \\
\hline 7.5 & 6.0 & 2.7 & $1.3 \times 10^{-6}$ & $5.0 \times 10^{-4}$ & MT1H & Metallothionein $1 \mathrm{H}$ \\
\hline 7.5 & 6.1 & 2.7 & $1.4 \times 10^{-10}$ & $3.8 \times 10^{-7}$ & PAQR5 & Progestin and adipoQ receptor family member V \\
\hline 4.2 & 2.8 & 2.7 & $1.7 \times 10^{-5}$ & $3.3 \times 10^{-3}$ & LINC00967 & Long intergenic non-protein coding RNA 967 \\
\hline 5.0 & 3.5 & 2.8 & $2.0 \times 10^{-7}$ & $1.0 \times 10^{-4}$ & $\mathrm{DAB} 2 ; \mathrm{C} 9$ & $\begin{array}{l}\text { Dab, mitogen-responsive phosphoprotein, } \\
\text { homolog } 2 \text { (Drosophila); complement component } 9\end{array}$ \\
\hline 4.8 & 3.3 & 2.8 & $3.0 \times 10^{-4}$ & $2.4 \times 10^{-2}$ & C17orf77 & Chromosome 17 open reading frame 77 \\
\hline 8.0 & 6.5 & 2.9 & $2.6 \times 10^{-6}$ & $8.0 \times 10^{-4}$ & SNORA75 & Small nucleolar RNA, H/ACA box 75 \\
\hline 8.0 & 6.5 & 2.9 & $5.0 \times 10^{-4}$ & $3.0 \times 10^{-2}$ & GBE1 & Glucan (1,4-alpha-), branching enzyme 1 \\
\hline 4.5 & 3.0 & 2.9 & $5.0 \times 10^{-4}$ & $3.0 \times 10^{-2}$ & TDGF1 & Teratocarcinoma-derived growth factor 1 \\
\hline 6.7 & 5.1 & 2.9 & $2.9 \times 10^{-7}$ & $2.0 \times 10^{-4}$ & RNF144A & Ring finger protein $144 \mathrm{~A}$ \\
\hline 6.2 & 4.6 & 3.0 & $7.7 \times 10^{-7}$ & $4.0 \times 10^{-4}$ & BAAT & Bile acid-CoA:amino acid $\mathrm{N}$-acyltransferase \\
\hline 7.3 & 5.7 & 3.0 & $5.1 \times 10^{-7}$ & $3.0 \times 10^{-4}$ & KDM3A & Lysine $(\mathrm{K})$-specific demethylase $3 \mathrm{~A}$ \\
\hline 7.2 & 5.6 & 3.0 & $5.6 \times 10^{-8}$ & $4.7 \times 10^{-5}$ & FAM13A & Family with sequence similarity 13 , member A \\
\hline 8.0 & 6.4 & 3.1 & $9.8 \times 10^{-9}$ & $1.2 \times 10^{-5}$ & ANPEP & Alanyl (membrane) aminopeptidase \\
\hline 7.2 & 5.5 & 3.1 & $8.8 \times 10^{-5}$ & $1.0 \times 10^{-2}$ & ALDOC & Aldolase C, fructosx 10-bisphosphate \\
\hline 4.5 & 2.9 & 3.1 & $1.3 \times 10^{-9}$ & $2.3 \times 10^{-6}$ & $\begin{array}{l}\text { MUC3A; } \\
\text { MUC3B }\end{array}$ & $\begin{array}{l}\text { Mucin 3A, cell surface associated; } \\
\text { mucin 3B, cell surface associated }\end{array}$ \\
\hline 10.5 & 8.9 & 3.1 & $4.5 \times 10^{-6}$ & $1.2 \times 10^{-3}$ & PIGR & Polymeric immunoglobulin receptor \\
\hline 6.3 & 4.7 & 3.1 & $3.0 \times 10^{-2}$ & $2.5 \times 10^{-1}$ & SPINK1 & Serine peptidase inhibitor, Kazal type 1 \\
\hline 8.7 & 7.0 & 3.1 & $6.5 \times 10^{-5}$ & $8.6 \times 10^{-3}$ & PLOD2 & Procollagen-lysine, 2 -oxoglutarate 5 -dioxygenase 2 \\
\hline 9.6 & 7.9 & 3.1 & $1.3 \times 10^{-5}$ & $2.8 \times 10^{-3}$ & SLC2A1 & $\begin{array}{l}\text { Solute carrier family } 2 \\
\text { (facilitated glucose transporter), member } 1\end{array}$ \\
\hline 6.1 & 4.5 & 3.1 & $4.2 \times 10^{-9}$ & $5.9 \times 10^{-6}$ & CDA & Cytidine deaminase \\
\hline 6.7 & 5.0 & 3.1 & $5.0 \times 10^{-4}$ & $2.9 \times 10^{-2}$ & FUT11 & Fucosyltransferase 11 (alpha $(1,3)$ fucosyltransferase) \\
\hline 8.3 & 6.6 & 3.1 & $8.7 \times 10^{-5}$ & $1.0 \times 10^{-2}$ & SLC6A19 & $\begin{array}{l}\text { Solute carrier family } 6 \\
\text { (neutral amino acid transporter), member } 19\end{array}$ \\
\hline 10.3 & 8.6 & 3.2 & $1.8 \times 10^{-6}$ & $6.0 \times 10^{-4}$ & BNIP3 & BCL2/adenovirus E1B 19kDa interacting protein 3 \\
\hline 9.3 & 7.6 & 3.2 & $1.0 \times 10^{-4}$ & $1.2 \times 10^{-2}$ & P4HA1 & Prolyl 4-hydroxylase, alpha polypeptide I \\
\hline 7.1 & 5.4 & 3.3 & $5.4 \times 10^{-6}$ & $1.4 \times 10^{-3}$ & PFKFB3 & 6-phosphofructo-2-kinase/fructosx 10-2,6-biphosphatase 3 \\
\hline
\end{tabular}

Table II. Continued 
Table II. Continued

\begin{tabular}{|c|c|c|c|c|c|c|}
\hline $\begin{array}{l}\text { GPI KO } \\
\text { Avg }(\log 2)\end{array}$ & $\begin{array}{c}\text { Ctrl } \\
\text { Avg }(\log 2)\end{array}$ & $\begin{array}{l}\text { Fold } \\
\text { change }\end{array}$ & $p$-Value & $\begin{array}{c}\text { FDR } \\
p \text {-Value }\end{array}$ & $\begin{array}{l}\text { Gene } \\
\text { symbol }\end{array}$ & Description \\
\hline 5.7 & 4.0 & 3.3 & $1.4 \times 10^{-8}$ & $1.5 \times 10^{-5}$ & GUCY2C & Guanylate cyclase $2 \mathrm{C}$ \\
\hline 6.3 & 4.6 & 3.3 & $2.7 \times 10^{-7}$ & $2.0 \times 10^{-4}$ & ANKRD37 & Ankyrin repeat domain 37 \\
\hline 6.6 & 4.9 & 3.3 & $9.4 \times 10^{-9}$ & $1.2 \times 10^{-5}$ & PMP22 & Peripheral myelin protein 22 \\
\hline 5.7 & 3.9 & 3.3 & $1.7 \times 10^{-7}$ & $1.0 \times 10^{-4}$ & BTNL3 & Butyrophilin-like 3 \\
\hline 5.0 & 3.3 & 3.4 & $1.2 \times 10^{-3}$ & $5.0 \times 10^{-2}$ & LRRC19 & Leucine rich repeat containing 19 \\
\hline 8.0 & 6.2 & 3.4 & $2.6 \times 10^{-7}$ & $2.0 \times 10^{-4}$ & ARRDC4 & Arrestin domain containing 4 \\
\hline 5.6 & 3.9 & 3.4 & $7.4 \times 10^{-9}$ & $9.6 \times 10^{-6}$ & PPARGC1A & $\begin{array}{l}\text { Peroxisome proliferator-activated } \\
\text { receptor gamma, coactivator } 1 \text { alpha }\end{array}$ \\
\hline 6.6 & 4.8 & 3.4 & $5.4 \times 10^{-7}$ & $3.0 \times 10^{-7}$ & TFF3 & Trefoil factor 3 \\
\hline 6.1 & 4.3 & 3.5 & $1.0 \times 10^{-7}$ & $7.3 \times 10^{-5}$ & RHOBTB3 & Rho-related BTB domain containing 3 \\
\hline 6.8 & 5.0 & 3.6 & $5.0 \times 10^{-4}$ & $2.8 \times 10^{-2}$ & SULT1C2 & Sulfotransferase family $1 \mathrm{C}$ member 2 \\
\hline 5.8 & 4.0 & 3.6 & $1.0 \times 10^{-8}$ & $1.2 \times 10^{-5}$ & CPXM2 & Carboxypeptidase X (M14 family), member 2 \\
\hline 5.8 & 3.9 & 3.6 & $1.9 \times 10^{-10}$ & $4.7 \times 10^{-7}$ & S100A9 & S100 calcium binding protein A9 \\
\hline 6.9 & 5.1 & 3.7 & $1.5 \times 10^{-11}$ & $6.2 \times 10^{-8}$ & SEMA6A & $\begin{array}{l}\text { Sema domain, transmembrane domain } \\
\text { (TM), and cytoplasmic domain, (semaphorin) } 6 \mathrm{~A}\end{array}$ \\
\hline 4.8 & 2.9 & 3.7 & $1.9 \times 10^{-6}$ & $7.0 \times 10^{-4}$ & PAG1 & $\begin{array}{l}\text { Phosphoprotein membrane anchor with } \\
\text { glycosphingolipid microdomains } 1\end{array}$ \\
\hline 6.9 & 4.9 & 3.8 & $1.6 \times 10^{-3}$ & $5.9 \times 10^{-2}$ & C6orf223 & Chromosome 6 open reading frame 223 \\
\hline 6.9 & 4.9 & 3.9 & $1.1 \times 10^{-8}$ & $1.3 \times 10^{-5}$ & FXYD4 & FXYD domain containing ion transport regulator 4 \\
\hline 9.5 & 7.5 & 3.9 & $2.0 \times 10^{-4}$ & $1.5 \times 10^{-2}$ & TFF1 & Trefoil factor 1 \\
\hline 4.9 & 2.9 & 4.1 & $1.6 \times 10^{-5}$ & $3.3 \times 10^{-3}$ & TGFBI & Transforming growth factor, beta-induced, $68 \mathrm{kDa}$ \\
\hline 9.0 & 6.8 & 4.3 & $2.1 \times 10^{-5}$ & $4.0 \times 10^{-3}$ & LCN15 & Lipocalin 15 \\
\hline 7.2 & 5.1 & 4.4 & $1.2 \times 10^{-6}$ & $5.0 \times 10^{-6}$ & TFF2 & Trefoil factor 2 \\
\hline 5.1 & 2.9 & 4.6 & $1.0 \times 10^{-6}$ & $4.0 \times 10^{-4}$ & TMEM45A & Transmembrane protein $45 \mathrm{~A}$ \\
\hline 5.5 & 3.2 & 4.9 & $8.3 \times 10^{-12}$ & $5.0 \times 10^{-8}$ & MUC17 & Mucin 17, cell surface associated \\
\hline 7.2 & 5.0 & 4.9 & $2.6 \times 10^{-3}$ & $7.5 \times 10^{-2}$ & IL37 & Interleukin 37 \\
\hline 5.2 & 2.7 & 5.4 & $1.2 \times 10^{-6}$ & $5.0 \times 10^{-4}$ & CASP5 & Caspase 5 \\
\hline 6.8 & 4.3 & 5.9 & $6.7 \times 10^{-6}$ & $1.7 \times 10^{-3}$ & NDRG1 & $\mathrm{N}$-myc downstream regulated 1 \\
\hline 7.0 & 4.0 & 8.1 & $1.4 \times 10^{-14}$ & $1.7 \times 10^{-10}$ & IRF8 & Interferon regulatory factor 8 \\
\hline 9.9 & 6.7 & 8.8 & $5.8 \times 10^{-9}$ & $7.8 \times 10^{-6}$ & HMGCS2 & $\begin{array}{l}\text { 3-hydroxy-3-methylglutaryl-CoA } \\
\text { synthase } 2 \text { (mitochondrial) }\end{array}$ \\
\hline 6.3 & 3.0 & 9.5 & $2.2 \times 10^{-10}$ & $5.1 \times 10^{-7}$ & HTR1D & $\begin{array}{l}\text { 5-hydroxytryptamine (serotonin) } \\
\text { receptor } 1 \mathrm{D}, \mathrm{G} \text { protein-coupled }\end{array}$ \\
\hline 6.9 & 3.3 & 11.9 & $1.4 \times 10^{-3}$ & $5.4 \times 10^{-2}$ & LOC 105378283 & $\begin{array}{l}\text { Putative ankyrin repeat domain-containing } \\
\text { protein ENSP00000383069 }\end{array}$ \\
\hline 9.1 & 4.2 & 29.5 & $1.3 \times 10^{-6}$ & $5.0 \times 10^{-4}$ & KIT & $\begin{array}{l}\text { v-kit Hardy-Zuckerman } 4 \text { feline sarcoma } \\
\text { viral oncogene homolog }\end{array}$ \\
\hline 9.4 & 3.2 & 72.2 & $9.4 \times 10^{-18}$ & $4.5 \times 10^{-13}$ & S100A7 & S100 calcium binding protein A7 \\
\hline
\end{tabular}

(ACC) (which decreases malonyl-CoA), causing the release of carnitine palmitoyltransferase 1A (CPT-1A), aiding the transport of fatty-acyl chains to mitochondria for $\beta$-oxidation (25-27). Theoretically, glycolysis would trigger elevation in $P G C-1 \alpha$, where this metabolic fuel switch should coincide with attenuation of biosynthesized steroids and cholesterol, where inactivation of HMG-CoA reductase (HMGCR) would then enable HMG-CoA to be rerouted to the production of ketones (this involving HMGCS2), both elevated in the $\mathrm{KO}$ clones. If this was the case, ketones $[\beta$-hydroxybutyrate (bHB), acetoacetate, acetone] would be produced through 2 or more acetyl-CoA molecules being condensed by the following enzymes: acetoacetyl-CoA thiolase (ACAT1), HMGCS2 and HMG-CoA lyase (HMGC). We did a follow up investigation for the presence of bHB and acetoacetate, finding no evidence of accumulation of both of these ketones (data not shown). Ketones, if present could be used to fuel the TCA cycle, through the reverse conversion of bHB to acetoacetate, then converted to acetoacetyl-CoA (by succinyl-CoA:3-ketoacid-CoA transferase, SCOT) and lastly to Acetyl-CoA by ACAT1. The end goal of a metabolic flip switch would be to regulate sources of acetyl-CoA to fuel the TCA cycle from glucose (during a fed state) to fats in a nonfed state ( $\beta$-oxidation). Another metabolic reprogramming gene that was elevated in both $\mathrm{KO}$ clones was acetyl-CoA acyltransferase 2 (ACAA2).

Future work will be required to corroborate if what we are seeing in both KOs is similar to lipid metabolic oncogenic 
A

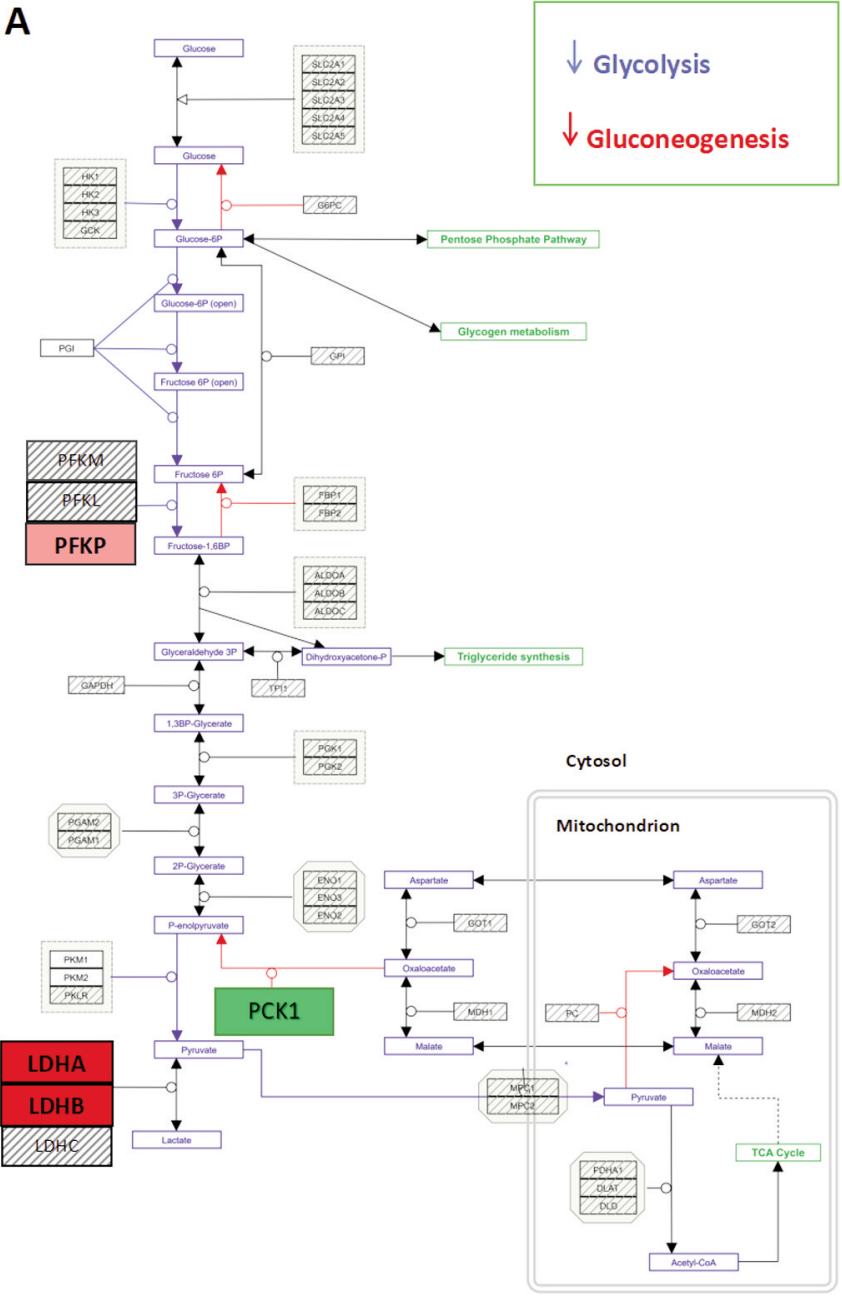

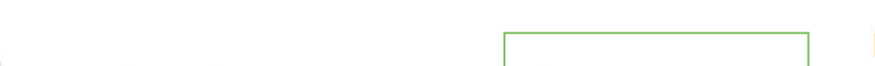

B

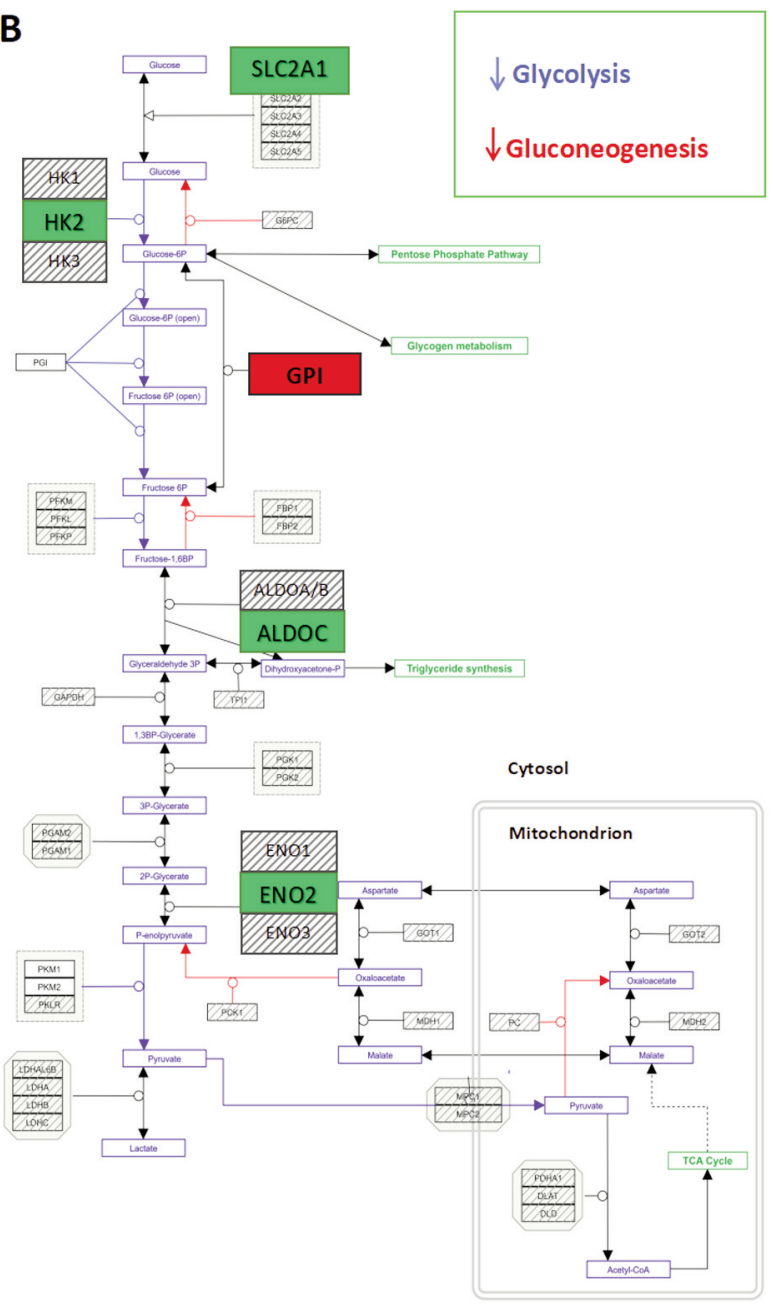

Figure 4. (A) Wiki Pathway analysis of LDHA/B DKO DEF changes observed in glycolysis, gluconeogenesis and mitochondrial TCA cycle pathways. (B) Wiki Pathway analysis of GPI KO DEF changes observed in glycolysis, gluconeogenesis and mitochondrial TCA cycle pathways. In red are the down-regulated transcripts and in green the up-regulated transcripts. Filtered by fold change $<-2$ or $>2$, p-Value $<0.05$.

reprogramming described in diverse cancers which involve the overexpression of both $H M G C 2(28,29)$ and $P G C-1 \alpha$ transcripts (24). Overexpression of $H M G C 2 / P G C-1 \alpha$ has been observed in high-grade colorectal cancer (30), colitisassociated cancer (31), clear cell renal cell cancer $(32,33)$, ovarian (34), liver (35), oral squamous cell carcinoma (36) and high-grade prostate cancer (37), and is reflective of recurrence and poor prognosis (38). The dual induction of $H M G C S 2 / P G C-1 \alpha$ also propels oncogenic mediated ketogenic events within the cancer milieu/stroma (for example, in cancer-associated fibroblasts) (39).

Increase in thioredoxin interacting protein transcripts $(T X N I P)$. Concomitant changes to glycolysis in both KOs were few, but the elevated expression of TXNIP was however notable (40). This is a pathological feature of type II diabetes and biological tissues that are unable to utilize glucose (glucose intolerance) (41). An earlier work with a fibroblast mutant defective in phosphoglucose isomerase (PGI) demonstrated that glucose-6-phosphate is the key accumulated metabolite "repressing" glucose transport activity (42), a mechanism further elucidated by Ayer's group in which TXNIP was shown to be induced through a nuclear transcription factor complex, ChREBP/Mlx, (MondoA-Mlx) (43). Finally, TXNIP, by direct interaction with GLUT1 transporter stops its activity by internalization through clathrin coated pits (44). Research using diverse models show a reciprocal self-perpetuated elevation in TXNIP, resulting in the inability to use glucose, with elevated glucose levels driving then further expression of TXNIP (40). The rise in TXNIP, 
Table III. DEG patterns in both KO clones relative to WT Controls.

\begin{tabular}{|c|c|c|c|c|c|c|c|}
\hline \multicolumn{2}{|c|}{$\begin{array}{l}\text { DEG patterns in both KO clones } \\
\text { relative to WT Controls }\end{array}$} & \multicolumn{3}{|c|}{ Effects in LDHA/B DKO } & \multicolumn{3}{|c|}{ Effects in GPI KO } \\
\hline $\begin{array}{l}\text { Gene } \\
\text { symbol }\end{array}$ & Description & FC & $p$-Value & $\begin{array}{c}\text { FDR } \\
p \text {-Value }\end{array}$ & FC & $p$-Value & $\begin{array}{c}\text { FDR } \\
p \text {-Value }\end{array}$ \\
\hline MUC6 & $\begin{array}{l}\text { Mucin 6, oligomeric mucus/ } \\
\text { gel-forming }\end{array}$ & -15.5 & $2.8 \times 10^{-12}$ & $1.0 \times 10^{-8}$ & -2.3 & $1.3 \times 10^{-2}$ & $1.7 \times 10^{-1}$ \\
\hline DKK4 & $\begin{array}{l}\text { Dickkopf WNT signaling pathway } \\
\text { inhibitor } 4\end{array}$ & -11.0 & $7.5 \times 10^{-8}$ & $3.5 \times 10^{-5}$ & -20.6 & $2.1 \times 10^{-8}$ & $2.0 \times 10^{-5}$ \\
\hline BGN & Biglycan & -7.3 & $1.0 \times 10^{-4}$ & $1.2 \times 10^{-2}$ & -8.4 & $3.0 \times 10^{-4}$ & $2.2 \times 10^{-2}$ \\
\hline $\mathrm{DSC}_{3}$ & Desmocollin 3 & -5.7 & $1.7 \times 10^{-12}$ & $9.0 \times 10^{-9}$ & -5.9 & $1.0 \times 10^{-11}$ & $5.3 \times 10^{-8}$ \\
\hline CRYBB1 & Crystallin beta B1 & -5.6 & $8.2 \times 10^{-7}$ & $2.0 \times 10^{-4}$ & -3.1 & $2.0 \times 10^{-4}$ & $1.5 \times 10^{-2}$ \\
\hline AKR1C2 & $\begin{array}{l}\text { Aldo-keto reductase family } 1 \text {, } \\
\text { member } \mathrm{C} 2\end{array}$ & -5.4 & $1.2 \times 10^{-9}$ & $1.4 \times 10^{-6}$ & -7.6 & $2.5 \times 10^{-10}$ & $5.5 \times 10^{-7}$ \\
\hline AKR1B10 & $\begin{array}{c}\text { Aldo-keto reductase family } 1, \text { member } \\
\text { B10 (aldose reductase) }\end{array}$ & -4.7 & $1.9 \times 10^{-13}$ & $4.7 \times 10^{-9}$ & -3.4 & $1.3 \times 10^{-10}$ & $3.7 \times 10^{-7}$ \\
\hline HULC & $\begin{array}{c}\text { Hepatocellular carcinoma up-regulated } \\
\text { long non-coding RNA }\end{array}$ & -3.8 & $1.7 \times 10^{-6}$ & $4.0 \times 10^{-4}$ & -3.9 & $5.6 \times 10^{-6}$ & $1.5 \times 10^{-3}$ \\
\hline BCL11A & $\begin{array}{l}\text { B-cell CLL/lymphoma } 11 \mathrm{~A} \\
\text { (zinc finger protein) }\end{array}$ & -3.5 & $2.8 \times 10^{-10}$ & $4.3 \times 10^{-7}$ & -2.3 & $2.5 \times 10^{-6}$ & $8.0 \times 10^{-4}$ \\
\hline HUNK & $\begin{array}{l}\text { Hormonally up-regulated Neu- } \\
\text { associated kinase }\end{array}$ & -3.4 & $3.5 \times 10^{-6}$ & $7.0 \times 10^{-4}$ & $\mid-4.2$ & $6.3 \times 10^{-7}$ & $3.0 \times 10^{-4}$ \\
\hline THSD4 & $\begin{array}{l}\text { Thrombospondin type } 1 \text { domain } \\
\text { containing } 4\end{array}$ & -3.3 & $7.0 \times 10^{-9}$ & $5.7 \times 10^{-6}$ & -2.9 & $1.3 \times 10^{-7}$ & $8.8 \times 10^{-5}$ \\
\hline HBE1 & Hemoglobin, epsilon 1 & -3.2 & $1.4 \times 10^{-5}$ & $2.0 \times 10^{-3}$ & -3.2 & $5.0 \times 10^{-6}$ & $1.4 \times 10^{-3}$ \\
\hline CALB2 & Calbindin 2 & -3.1 & $2.9 \times 10^{-5}$ & $3.6 \times 10^{-3}$ & -7.8 & $1.4 \times 10^{-6}$ & $5.0 \times 10^{-4}$ \\
\hline $\mathrm{ALDH}_{3} \mathrm{~A}_{1}$ & $\begin{array}{l}\text { Aldehyde dehydrogenase } 3 \text { family, } \\
\text { member A1 }\end{array}$ & -3.0 & $3.7 \times 10^{-6}$ & $8.0 \times 10^{-4}$ & -3.6 & $7.4 \times 10^{-6}$ & $1.8 \times 10^{-3}$ \\
\hline FGFBP1 & $\begin{array}{l}\text { Fibroblast growth factor binding } \\
\text { protein } 1\end{array}$ & -2.9 & $5.4 \times 10^{-7}$ & $2.0 \times 10^{-4}$ & -2.1 & $2.2 \times 10^{-5}$ & $4.0 \times 10^{-3}$ \\
\hline OR51B4 & $\begin{array}{l}\text { Olfactory receptor, family } 51 \text {, } \\
\text { subfamily B, member } 4\end{array}$ & -2.7 & $7.0 \times 10^{-4}$ & $3.4 \times 10^{-2}$ & -3.1 & $5.0 \times 10^{-4}$ & $3.0 \times 10^{-2}$ \\
\hline EIF2B5-AS1 & EIF2B5 antisense RNA 1 & -2.7 & $6.0 \times 10^{-4}$ & $2.9 \times 10^{-2}$ & -3.9 & $1.3 \times 10^{-5}$ & $2.9 \times 10^{-3}$ \\
\hline GPX2 & Glutathione peroxidase 2 & -2.7 & $1.5 \times 10^{-5}$ & $2.2 \times 10^{-3}$ & -4.5 & $1.8 \times 10^{-6}$ & $6.0 \times 10^{-4}$ \\
\hline GPX8 & Glutathione peroxidase 8 (putative) & -2.7 & $6.3 \times 10^{-7}$ & $2.0 \times 10^{-4}$ & -2.5 & $3.1 \times 10^{-6}$ & $1.0 \times 10^{-3}$ \\
\hline ANOS1 & Anosmin 1 & -2.7 & $5.3 \times 10^{-9}$ & $4.6 \times 10^{-6}$ & -2.6 & $2.1 \times 10^{-8}$ & $2.0 \times 10^{-5}$ \\
\hline SLC7A11 & $\begin{array}{c}\text { Solute carrier family } 7 \text { (AA transporter } \\
\text { LC, xc- system), member } 11\end{array}$ & -2.7 & $8.5 \times 10^{-6}$ & $1.4 \times 10^{-3}$ & -2.3 & $7.5 \times 10^{-5}$ & $9.2 \times 10^{-3}$ \\
\hline VCAN & Versican & -2.7 & $9.7 \times 10^{-3}$ & $1.6^{\times 10-1}$ & -10.7 & $1.3 \times 10^{-5}$ & $2.8 \times 10^{-3}$ \\
\hline TFCP2 & Transcription factor $\mathrm{CP} 2$ & -2.7 & $2.6 \times 10^{-9}$ & $2.7 \times 10^{-6}$ & -2.0 & $1.8 \times 10^{-6}$ & $6.0 \times 10^{-4}$ \\
\hline
\end{tabular}




\begin{tabular}{|c|c|c|c|c|c|c|c|}
\hline \multicolumn{2}{|c|}{$\begin{array}{l}\text { DEG patterns in both KO clones } \\
\text { relative to WT Controls }\end{array}$} & \multicolumn{3}{|c|}{ Effects in LDHA/B DKO } & \multicolumn{3}{|c|}{ Effects in GPI KO } \\
\hline $\begin{array}{l}\text { Gene } \\
\text { symbol }\end{array}$ & Description & FC & $p$-Value & $\begin{array}{c}\text { FDR } \\
p \text {-Value }\end{array}$ & FC & $p$-Value & $\begin{array}{c}\text { FDR } \\
p \text {-Value }\end{array}$ \\
\hline $\begin{array}{l}\text { CES1; } \\
\text { CES1P1 }\end{array}$ & $\begin{array}{c}\text { Carboxylesterase } 1 \text {; carboxylesterase } 1 \\
\text { pseudogene } 1\end{array}$ & -2.6 & $5.5 \times 10^{-6}$ & $1.0 \times 10^{-3}$ & -2.0 & $3.0 \times 10^{-4}$ & $2.4 \times 10^{-2}$ \\
\hline $\mathrm{ALDH} 1 \mathrm{~A} 1$ & $\begin{array}{c}\text { Aldehyde dehydrogenase } 1 \text { family, } \\
\text { member } A 1\end{array}$ & -2.6 & $1.1 \times 10^{-8}$ & $7.8 \times 10^{-6}$ & -2.8 & $2.0 \times 10^{-8}$ & $2.0 \times 10^{-5}$ \\
\hline LYZ & Lysozyme & -2.6 & $1.8 \times 10^{-8}$ & $1.2 \times 10^{-5}$ & -2.0 & $.1 \times 10^{-6}$ & $1.4 \times 10^{-3}$ \\
\hline CORO1A & Coronin, actin binding protein, $1 \mathrm{~A}$ & -2.2 & $1.0 \times 10^{-4}$ & $9.6 \times 10^{-3}$ & -2.1 & $0 \times 10^{-3}$ & $8.0 \times 10^{-2}$ \\
\hline PLA2G16 & Phospholipase A2, group XVI & -2.2 & $7.0 \times 10^{-9}$ & $5.7 \times 10^{-6}$ & -2.5 & $3.0 \times 10^{-9}$ & $4.5 \times 10^{-6}$ \\
\hline$A K R_{1} C_{1}$ & $\begin{array}{l}\text { Aldo-keto reductase family } 1 \text {, member } \\
\mathrm{C}_{1}\end{array}$ & -2.1 & $6.4 \times 10^{-6}$ & $1.1 \times 10^{-3}$ & -2.5 & $6.6 \times 10^{-7}$ & $3.0 x$ \\
\hline HOXB9 & Homeobox B9 & -2.1 & $5.2 \times 10^{-6}$ & $1.0 \times 10^{-3}$ & -4.7 & $5.0 \times 10^{-11}$ & $1.7 \times 10^{-7}$ \\
\hline SERPINB5 & $\begin{array}{l}\text { Serpin peptidase inhibitor, clade B } \\
\text { (ovalbumin), member } 5\end{array}$ & -2.1 & $1.4 \times 10^{-7}$ & $5.7 \times 10^{-5}$ & -2.1 & $4.6 \times 10^{-7}$ & $3.0 \times 1$ \\
\hline GXYLT2 & Glucoside xylosyltransferase 2 & -2.1 & $2.1 \times 10^{-6}$ & $5.0 \times 10^{-4}$ & -2.2 & $.7 \times 10^{-6}$ & $9.0 \times 10^{-4}$ \\
\hline APCDD1 & $\begin{array}{l}\text { Adenomatosis polyposis coli down- } \\
\text { regulated } 1\end{array}$ & -2.0 & $7.3 \times 10^{-6}$ & $1.3 \times 10^{-3}$ & -5.7 & $1.7 \times 10^{-10}$ & $4.3 \times 10^{-7}$ \\
\hline ACAA2 & Acetyl-CoA acyltransferase 2 & 1.7 & $9.2 \times 10^{-7}$ & $2.0 \times 10^{-4}$ & 1.7 & $1.2 \times 10^{-5}$ & $2.7 \times 10^{-3}$ \\
\hline TXNIP & Thioredoxi & 2.0 & $4.4 \times 10^{-8}$ & $2.3 \times 10^{-5}$ & 2.0 & $2.4 \times 10^{-6}$ & $8.0 \times 10^{-4}$ \\
\hline LYST & Lysosomal trafficking regulator & 2.0 & $2.6 \times 10^{-3}$ & $7.8 \times 10^{-2}$ & 2.3 & $1.4 \times 10^{-5}$ & $2.1 \times 10^{-3}$ \\
\hline ARRDC4 & Arrestin domain containing 4 & 2.1 & $3.0 \times 10^{-6}$ & $6.0 \times 10^{-4}$ & 3.4 & $2.6 \times 10^{-7}$ & $2.0 \times 10^{-4}$ \\
\hline SECTM1 & Secreted and transmembrane 1 & 2.2 & $1.8 \times 10^{-5}$ & $2.5 \times 10^{-3}$ & 2.3 & $3.0 \times 10^{-4}$ & $2.3 \times 10^{-2}$ \\
\hline C6orf222 & $\begin{array}{l}\text { Chromosome } 6 \text { open reading frame } \\
\qquad 222\end{array}$ & 2.3 & $3.0 \times 10^{-4}$ & $2.0 \times 10^{-2}$ & 2.5 & $3.7 \times 10^{-3}$ & $9.0 \times 10^{-2}$ \\
\hline HMGCS2 & $\begin{array}{l}\text { 3-hydroxy-3-methylglutaryl-CoA } \\
\text { synthase } 2 \text { (mitochondrial) }\end{array}$ & 2.4 & $5.4 \times 10^{-6}$ & $1.0 \times 10^{-3}$ & 8.8 & $5.8 \times 10^{-9}$ & $7.8 \times 10^{-6}$ \\
\hline ZSCAN12P1 & $\begin{array}{l}\text { Zinc finger and SCAN domain } \\
\text { containing } 12 \text { pseudogene } 1\end{array}$ & 2.5 & $3.6 \times 10^{-8}$ & $2.0 \times 10^{-5}$ & 2.1 & $1.2 \times 10^{-6}$ & $5.0 \times 10^{-4}$ \\
\hline SEMA6A & $\begin{array}{l}\text { Sema domain, trans mem (TM), } \\
\text { cytoplasmic , (semaphorin) 6A }\end{array}$ & 2.6 & $5.2 \times 10^{-10}$ & $7.2 \times 10^{-7}$ & 3.7 & $1.5 \times 10^{-11}$ & $6.2 \times 10^{-8}$ \\
\hline SNORA75 & nucleolar RNA, H/ACA box 75 & 2.7 & $1.1 \times 10^{-7}$ & $4.7 \times 10^{-5}$ & 2.9 & $2.6 \times 10^{-6}$ & $8.0 \times 10^{-4}$ \\
\hline CD68 & CD68 molecule & 3.3 & $9.2 \times 10^{-8}$ & $4.2 \times 10^{-5}$ & 2.5 & $7.5 \times 10^{-6}$ & $1.9 \times 10^{-3}$ \\
\hline PPARGC1A & $\begin{array}{l}\text { Peroxisome proliferator-activated } \\
\text { receptor gamma, coactivator } 1 \text { alpha }\end{array}$ & 3.4 & $3.8 \times 10^{-10}$ & $5.4 \times 10^{-7}$ & 3.4 & $7.4 \times 10^{-9}$ & $9.6 \times 10^{-6}$ \\
\hline IRF9 & Interferon regulatory factor 9 & 4.3 & $1.5 \times 10^{-8}$ & $1.1 \times 10^{-5}$ & 2.2 & $9.9 \times 10^{-3}$ & $1.5 \times 10^{-1}$ \\
\hline FXYD4 & $\begin{array}{c}\text { FXYD domain containing ion transport } \\
\text { regulator } 4\end{array}$ & 4.7 & $1.3 \times 10^{-10}$ & $2.3 \times 10^{-7}$ & 3.9 & $1.1 \times 10^{-8}$ & $1.3 \times 10^{-5}$ \\
\hline SPINK1 & Serine peptidase inhibitor, Kazal type 1 & 5.6 & $2.0 \times 10^{-4}$ & $1.3 \times 10^{-2}$ & 3.1 & $3.0 \times 10^{-2}$ & $2.5 \times 10^{-1}$ \\
\hline
\end{tabular}


besides a reduction in glucose uptake, can lead to reduced glycolytic enzyme activities (such as Phosphofructokinase, Pyruvate Kinase and Lactate Dehydrogenase), the Pentose phosphate pathway (PPP) and Glucose-6-Phosphate Dehydrogenase (45). This is entirely opposite in cancer, where a reported loss of TXNIP correlates to the observed Warburg effect: rapid glycolysis, higher glucose uptake, and activation of glutaminolysis $(46,47)$. In other words, diabetes is a disease involving higher TXNIP expression with the inability to use glucose, while cancer is a disease corresponding to lower TXNIP and heightened ability to use glucose, particularly with regards to glycolysis.

Reduction in aldehyde dehydrogenase transcripts (ALDHs) One of the most significant losses in response to glycolytic impairment in both $\mathrm{KO}$ clones was the concomitant repression of several aldehyde dehydrogenase transcripts, specifically $A L D H 1 A 1, A L D H 2$ (mitochondrial), and $A L D H 3 A 1$, which could create a non-ergogenic vulnerability to cancer cells. High expression of these genes is observed in many cancers, playing a critical role in the biosynthesis of retinoic acid (RA), nuclear signaling $(48,49)$ and contribution toward the "stemness" or self-renewal features which perpetuates tumor initiation and drug/radiation resistance (50-54). While ALDHs are keys in the collective stemness gene profile (CSN6, CD15, CD24, CD166, TRIM21, CD133, CD82, CD105, CD44,CD90, ALDH1A1, EPCAM, SOX2, SOX9, LGR5, BMI1, NANOG, OCT4, $C X C R 4$ and $C D 24)$, ALDHs are specifically up-regulated by pro-oncogenic proteins such as cytokines, growth factors or pollutants which trigger epithelial-mesenchymal transition (EMT), colony formation, migration, and metastasis in diverse cancers with poor clinical outcome (55-61).

One of the critical questions that comes to mind from this particular aspect of the data is the following: is there a correlation between the reduction in ALDHs and metabolic reprogramming? While few studies have investigated this aspect, there are reports suggesting that, in adipocytes, the observed overexpression of ALDH1A1 is associated with fatty acid synthesis, obesity, insulin resistance (62) as well as lipogenesis and triglyceride production (63). In cancer tissues, the rise in ALDHs is associated with heightened glucose uptake, activation of the glycolytic pathway, and elevation of GLUT1 (64). While the loss of ALDHs coincides with complete loss of glucose utilization in both $\mathrm{KO}$ clones, the causal effect, if any, between these two events will need further investigation. Future studies will be needed to confirm if LDHA/B inhibitors could be combined with ALDH inhibitors to evoke a more significant chemotherapeutic effect of drugs and radiation (48), and could be explored with ALDH inhibitors such as (-)epigallocatechin gallate $(65,66)$, rocaglamide A $(67)$, NCT501 (67), and ellagic acid (68).
Reduction in Aldo-keto reductase (AKR) transcripts. In this work, we also see a consistent downregulation of several $A R K s$, including $A K R 1 B 10, A K R 1 C 1, A K R 1 C 2$ and $A K R 1 C 3$ in both $\mathrm{KO}$ clones. Higher expression of $A R K S$ is frequently reported in cases of liver damage due to environmental pollutants (69), hepatitis C and B virus, aflatoxin B1 (70-72), nonalcoholic fatty liver, steatohepatitis (73), fibrosis (74), and cirrhosis (75). The ARKs, including AKR1B10 and AKRIC3, are also overexpressed in a number of premalignant and established cancers including liver cancer (76-78), and play a role in prostanoid synthesis which drives hormone-dependent cancers including prostate, breast, and endometrial ones (7981). Heightened $A K R 1 B 10$ overexpression is also critical to cancers associated with protein prenylation of mutant KRAS; which is a formidable prognostic indicator to tumor invasion, metastasis and progression (82-84), chemoresistance (85), radiation resistance (86), recurrence, tumor size and overall poor clinical outcomes (87-89). Downregulation of ARK1B10 observed in both glycolysis-null clones would favor a vulnerability to tumor growth. This has been shown many times, through either KO or inhibition of AKR1B10 in various in vitro and in vivo models which demarcates reduced tumor growth, metastasis $(90,91)$, protein prenylation (92), colony formation (3), and ultimately more significant response to chemotherapy drugs such as doxorubicin (93). Given this role in cancer, future research could be conducted to examine if there is a synergistic effect when using LDHA/B inhibitors and ARK class inhibitors, such as apigenin, luteolin (94), epalrestat (95), hop-derived compounds (adhumulone), nhumulone (96), xanthohumol isoxanthohumol (97), caffeic acid phenethyl esters, tolrestat, zopolrestat, sorbinil (76), curcumin, magnolol honokiol (16), oleanolic acid (76) and baccharin analogs or sulfonylureas (98).

WNT signaling. One of the most significant differential shifts in both KOs was the reduced expression of inhibitory controls over WNT signaling: loss of DKK4 (-20.53 FC), which otherwise would act as a canonical WNT/ $\beta$-catenin signaling inhibitor. In the $G P I \mathrm{KO}$, this corresponded to a significant elevation of $C$-KIT (+29.65 FC), a major driving force in $\mathrm{WNT} / \beta$-catenin signaling which plays a role in cancer stem cell reprogramming, EMT, immune evasion and drug resistance $(99,100)$. The effects of enhanced WNT

Figure 5. (A) Wiki Pathway analysis of LDHA/B DKO DEF changes observed in integrated amino acid metabolic pathways. (B) Wiki Pathway analysis of GPI KO DEF changes observed in integrated amino acid metabolic pathways. In red are the down-regulated transcripts and in green the up-regulated transcripts. Filtered by fold change $<-2$ or $>2$, $p$-Value $<0.05$. 

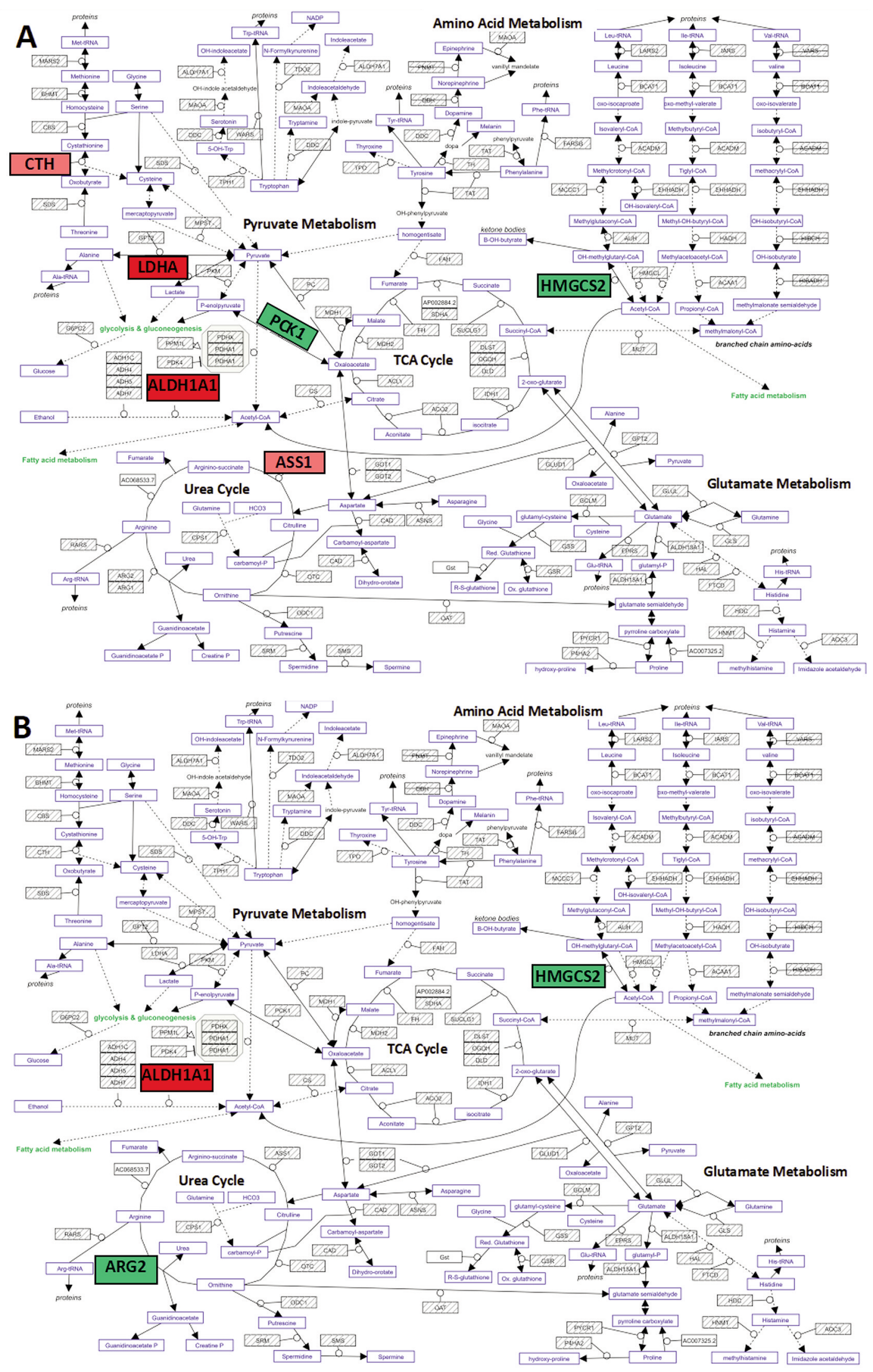
signaling on glycolysis-null cells would definitely deserve further investigation.

Normally, DKK4 acts to suppress the canonical WNT signaling as follows. Briefly, canonical WNT signaling is initiated by WNT ligands that bind to Frizzled (Fzd) receptor family, and cytoplasmic low-density-lipoproteinrelated protein 5/6 (LRP5/6, the binding target of DKK4) $(101,102)$. If DKK inhibits this mechanism, then it would prevent the phosphorylation, recruitment, and polymerization of Dishevelled (Dvl), that would otherwise bind axin to the receptor and inactivate the $\beta$-catenin destruction complex. When activated, this leads to a subsequent rise in $\beta$-catenin levels which precipitates its translocation into the nucleus to interact with transcription factors [T-cell factor (TCF) and lymphoid enhancer factor (LEF)] to promote cell proliferation and tumorigenesis (103). Both KOs also displayed reduced expression of $A P C$ down-regulated 1 (APCDD1), which also binds to WNT3a/LRP5 $(102,104)$ and inhibits WNT/ $\beta$-catenin LEF transcriptional signaling $(105,106)$. The attenuation of negative WNT controls (APCDD1 and $D K K 4$ ) in glycolytic KOs would, in theory, lead to a rise in $\beta$-catenin, which, if occurring in the cytoplasm, could interact with cadherin, bind to the actin filaments or TGF- $\beta$ and alter cell-cell adhesion, thus resulting in a very loose mesenchymal phenotype contributing to metastasis. This could be a negative consequence, particularly for colon cancer, since high catenin levels, coinciding with inability of proteolytic degradation of $\beta$-catenin as in the case of mutations in $\operatorname{TrCP1}(\beta$-TrCP) E3 ubiquitin ligase motifs or $\beta$-catenin recruiting motifs of adenomatous polyposis coli (APC) can lead to precancerous polyps with the capacity to transform into lethal tumors (107).

However, it is important to note that concerning DKK4, there is a remarkable inconsistency in regard to its effect on WNT signaling. While many reports reveal its tumor suppressor role, it is also reported to be overexpressed in diverse cancers, corresponding to tumorigenesis and metastasis (108) including esophageal cancer (109), endometrial cancer and thyroid carcinoma $(110,111)$. While healthy tissue is devoid of DKKs, elevated DKK expression is found in matched tumor tissues (112) and overexpressed chemoresistant $(113,114)$ and metastatic xenograft models of CRC (115). Further confounding its role, a reduction in DKK4, either experimentally manipulated or inherent, corresponds to increased $\beta$ catenin-mediated transcriptional activity on target genes including Axin2 and CCNDl (116), which drive proliferation (117). While several research groups are looking into this paradox, it is believed that pro-tumoral properties of DKK may be attributable to activating a noncanonical c-Jun $\mathrm{N}$-terminal kinase (JNK) signaling pathway while inhibiting the WNT-canonical pathway (118).
Future research will be required to evaluate the synergistic effects of LDHA/B inhibitors with a diverse range of drugs that target WNT signaling. C-KIT can be inhibited by drugs such as imatinib or mastinib which down-regulate stem cell markers, enhance the therapeutic efficacy of cytostatic agents (119), and prevent its role in the stroma to promote tumorigenesis, angiogenesis, proliferation and survival $(120,121)$.

Extracellular matrix (ECM). One of the most striking visual observations to the morphology of the knockouts was the loss of cell-cell adhesion relative to WT in both KO clones, also reflected by a significant loss of genes controlling cellcell/cell - surface or ECM systems. Some of these included losses to ECM component genes which would otherwise play a role in cancer migration, motility and invasion, such as Anosmin 1 or Biglycan (122-125). The type of losses in ECM anchoring related genes as shown in this study (such as $V C A N$ ) could evoke the remodeling of tumor stroma matrix which is known to enable sequestration of tumorpromoting immune cells and angiogenesis (126-128). Combined with losses in desmocollin $3(129,130)$ and moesin $(M S N)$ (131), it could then worsen EMT and metastasis (132).

In summary, this work provides an overall broad layout of the transcriptomic changes that are triggered by severe compromise to glycolysis by KO of $L D H A / B$ or GPI genes, specific to this highly aggressive type of colon cancer. Future research will be required to clearly demarcate all plausible ergogenic pathways in tumor cells to identify a multi-target tumor-specific approach to destroy the tumor metabolic requirements.

\section{Availability of Data and Material}

The dataset has been deposited to NIH Gene Expression Omnibus located at: https://www.ncbi.nlm.nih.gov/geo/query/acc.cgi?acc= GSE149289.

\section{Conflicts of Interest}

The Authors declare that they have no conflicts of interest.

\section{Authors' Contributions}

EM carried out the basic metabolic profiling and microarray work. $\mathrm{RB}$ assisted with daily tasks and consultation on experimental design and troubleshooting. NM assisted with ATP assays. KS oversaw this project from start to finish by scientific consultation, guidance, manuscript preparation and review. ZM isolated and characterized the two glycolytic-null cell lines. SC has done cell growth, viability, clonogenic assays and assisted manuscript preparation and edition. JP oversaw this collaborative project by scientific consultation, guidance and review. 


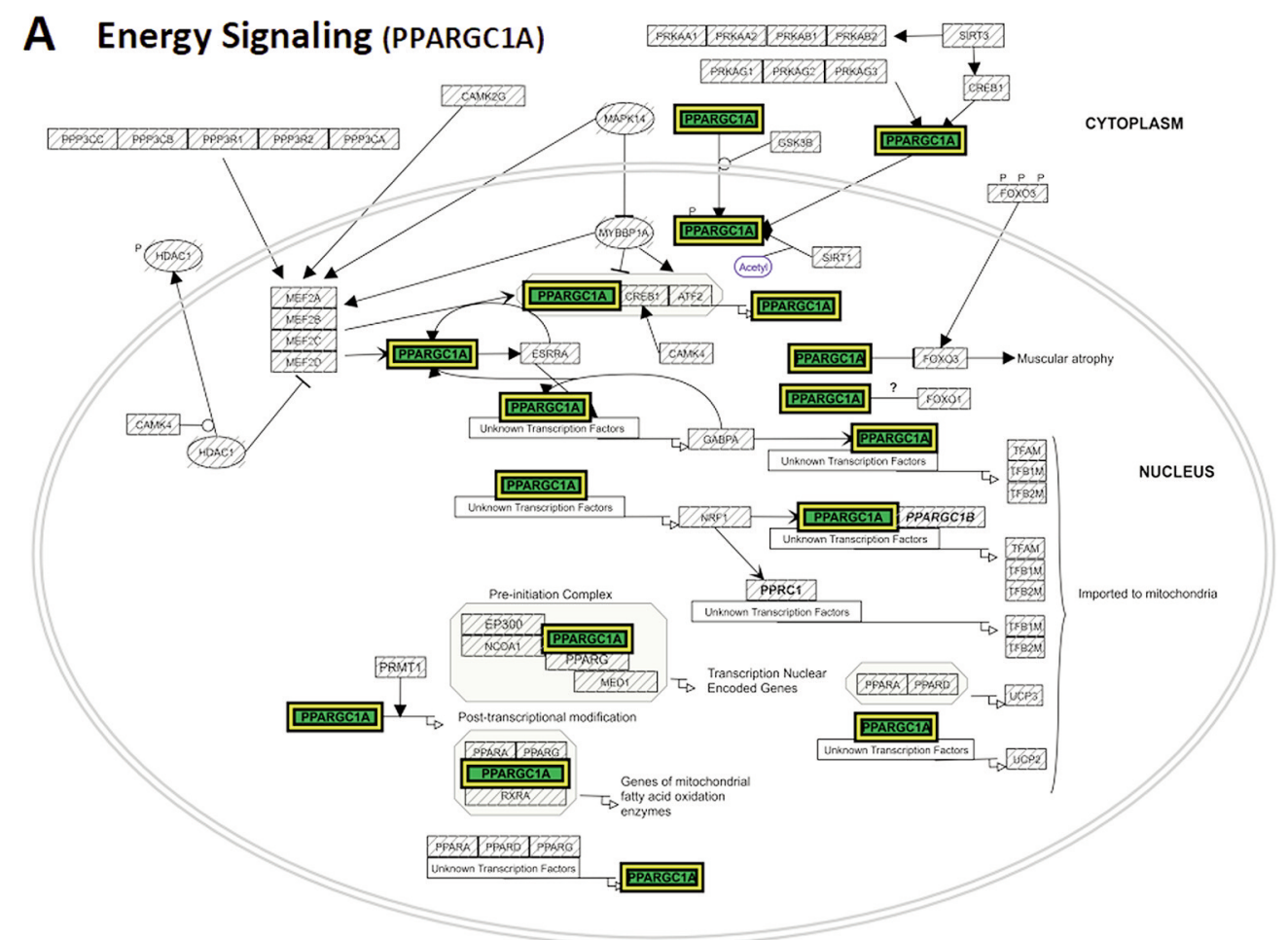

B

(HMG-CoA cycle)

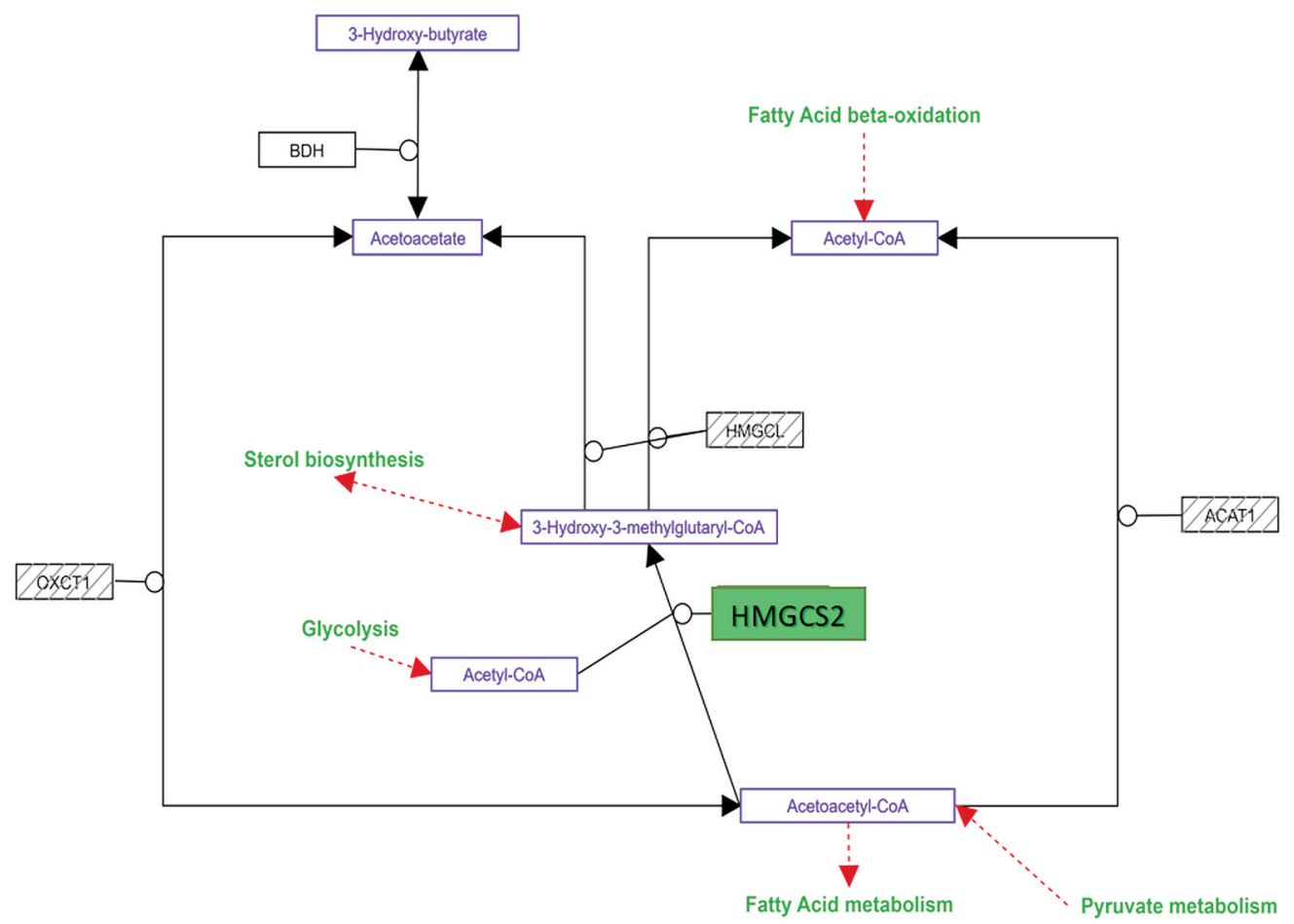

Figure 6. (A) Wiki Pathway analysis of overlapping upregulated DEG changes observed in both LDHA/B DKO and GPI KO in energy pathways. The PPARGC1A differential transcript changes were as follows: WT versus LDHA/B DKO: $(F C+3.43, F D R$-Value $<0.001)$; WT versus GPI KO: $(F C+3.39, F D R$ p-Value $<0.001)$. (B) Wiki Pathway analysis of overlapping up-regulated DEF changes observed in both LDHA/B DKO and GPI $K O$ in the HMG-CoA pathway. The HMGCS2 differential transcript changes were as follows: WT versus LDHA/B DKO: (FC +3.43, FDR pValue<0.001); WT versus GPI KO: $(F C+3.39, F D R$-Value $<0.001)$. In red are the down-regulated transcripts and in green the up-regulated transcripts. Filtered by fold change $<-2$ or $>2$, $p$-Value $<0.05$. 

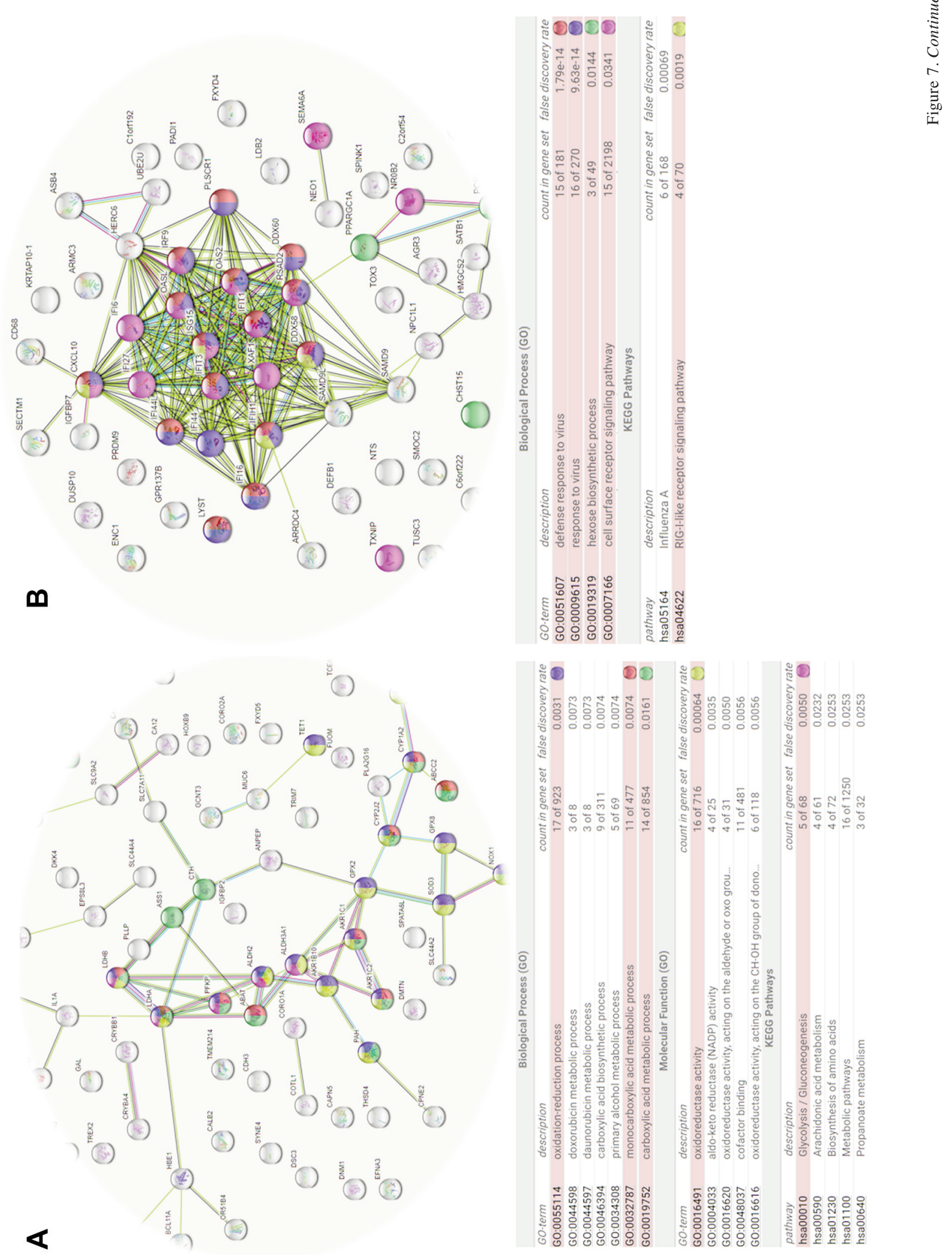

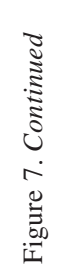

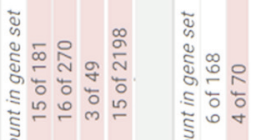

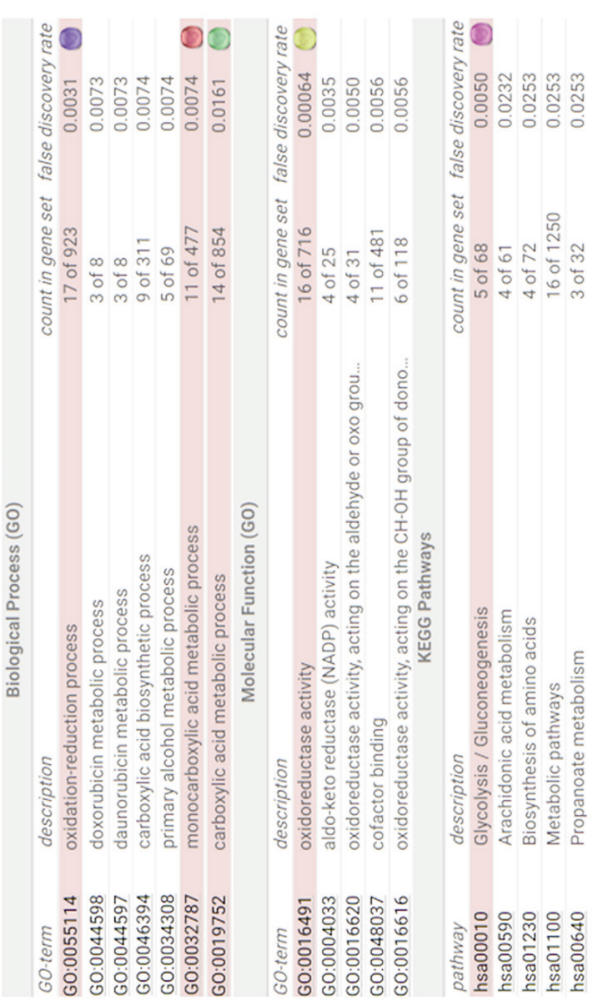



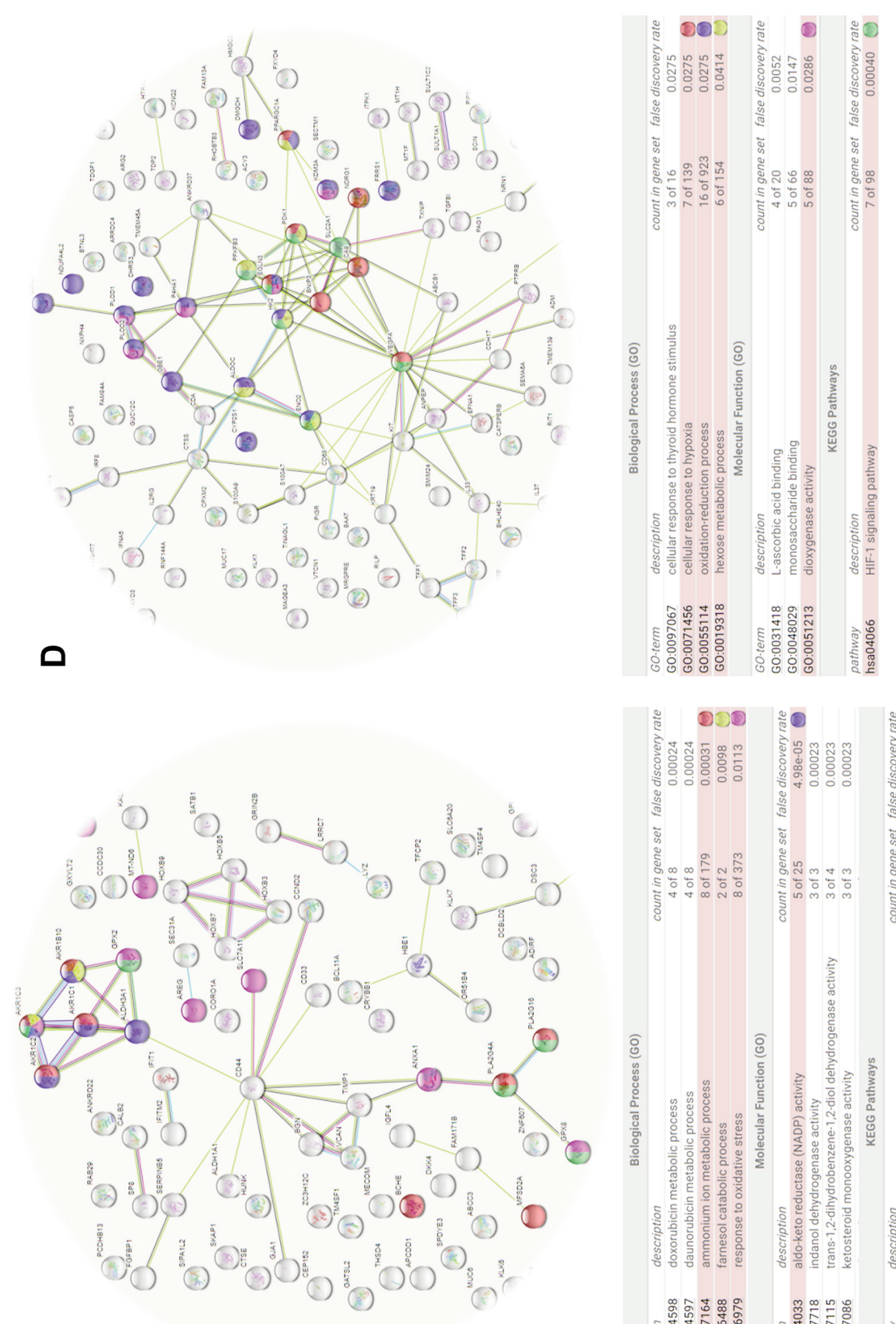

0

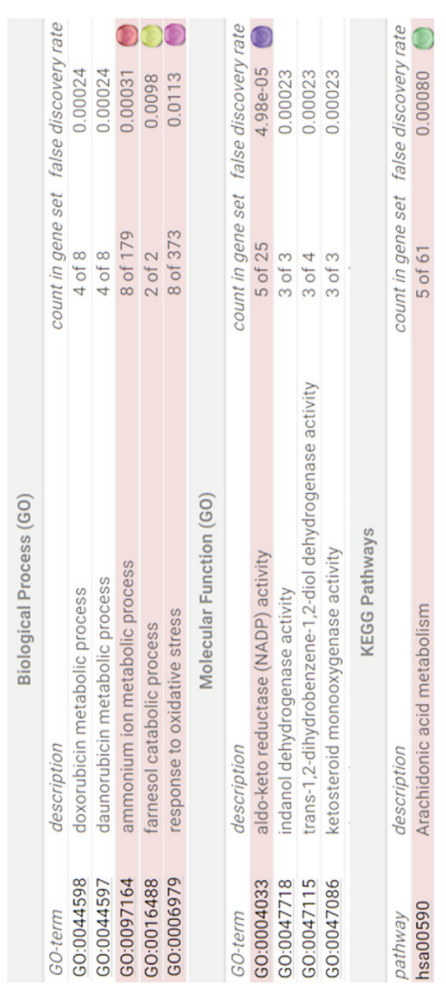

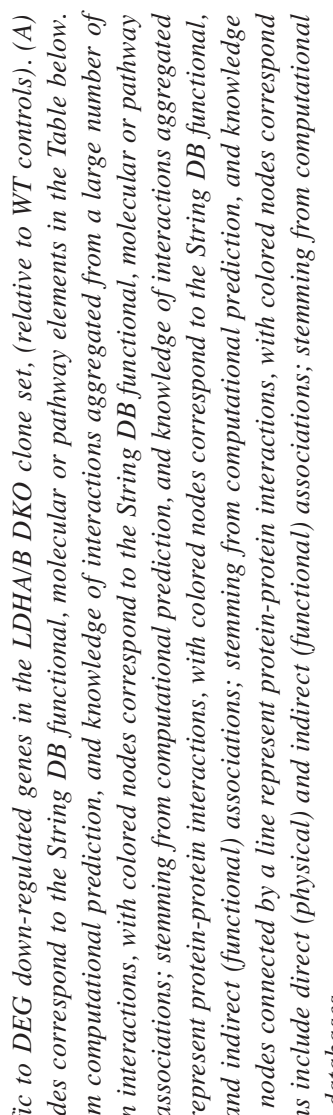

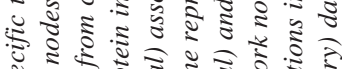

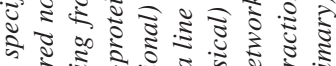

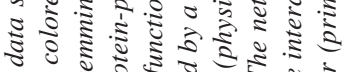

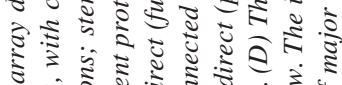
8.

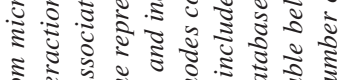

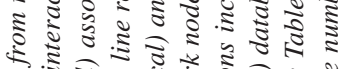

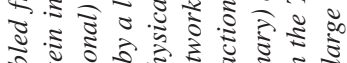

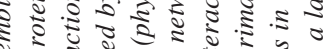

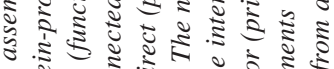

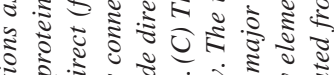

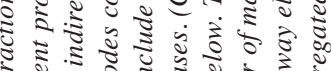

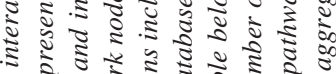

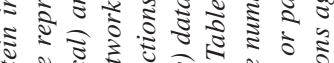

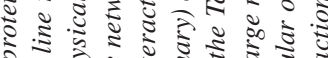

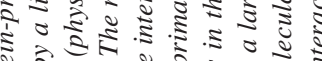

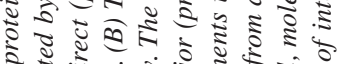

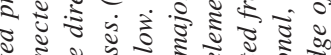

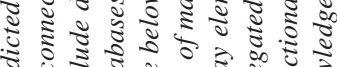

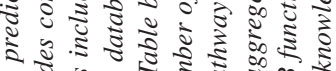

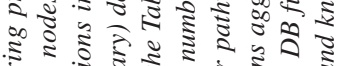

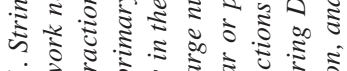
万.

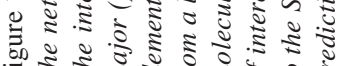




\section{Acknowledgements}

This research was supported by the National Institute of Minority Health and Health Disparities of the National Institutes of Health through Grant Number G12 MD007582 and Grant Number P20 MD006738 - (Dr J. Pouyssegur's group) Research was funded by University Côte d'Azur, IRCAN, CNRS and by the Centre Scientifique de Monaco and Donation from GEMLUC.

\section{References}

1 Kroemer G and Pouyssegur J: Tumor cell metabolism: Cancer's achilles' heel. Cancer Cell 13(6): 472-482, 2008. PMID: 18538731. DOI: 10.1016/j.ccr.2008.05.005

2 Cassim S, Vucetic M, Zdralevic M and Pouyssegur J: Warburg and beyond: The power of mitochondrial metabolism to collaborate or replace fermentative glycolysis in cancer. Cancers (Basel) 12(5), 2020. PMID: 32365833. DOI: 10.3390/cancers 12051119

3 Pillai SR, Damaghi M, Marunaka Y, Spugnini EP, Fais S and Gillies RJ: Causes, consequences, and therapy of tumors acidosis. Cancer Metastasis Rev 38(1-2): 205-222, 2019. PMID: 30911978. DOI: 10.1007/s10555-019-09792-7

4 da Silva VP, Mesquita CB, Nunes JS, de Bem Prunes B, Rados PV and Visioli F: Effects of extracellular acidity on resistance to chemotherapy treatment: A systematic review. Med Oncol 35(12): 161, 2018. PMID: 30377828. DOI: 10.1007/s12032-018-1214-4

5 Taddei ML, Pietrovito L, Leo A and Chiarugi P: Lactate in sarcoma microenvironment: Much more than just a waste product. Cells 9(2), 2020. PMID: 32102348. DOI: 10.3390/cells9020510

6 Parks SK, Mueller-Klieser W and Pouyssegur J: Lactate and acidity in the cancer microenvironment. Annu Rev Cancer Biol 4: 141-158, 2020. DOI: 10.1146/annurev-cancerbio-030419- 033556

7 Cassim S and Pouyssegur J: Tumor microenvironment: A metabolic player that shapes the immune response. Int J Mol Sci 21(1), 2019. PMID: 31881671. DOI: 10.3390/ijms21010157

8 Chiche J, Brahimi-Horn MC and Pouyssegur J: Tumour hypoxia induces a metabolic shift causing acidosis: A common feature in cancer. J Cell Mol Med 14(4): 771-794, 2010. PMID: 20015196. DOI: 10.1111/j.1582-4934.2009.00994.x

9 de la Cruz-Lopez KG, Castro-Munoz LJ, Reyes-Hernandez DO, Garcia-Carranca A and Manzo-Merino J: Lactate in the regulation of tumor microenvironment and therapeutic approaches. Front Oncol 9: 1143, 2019. PMID: 31737570. DOI: 10.3389/fonc.2019. 01143

10 Wei D, Engelman DM, Reshetnyak YK and Andreev OA: Mapping ph at cancer cell surfaces. Mol Imaging Biol 21(6): 1020-1025, 2019. PMID: 30989440. DOI: 10.1007/s11307-01901335-4

11 Wehr J, Sikorski EL, Bloch E, Feigman MS, Ferraro NJ, Baybutt TR, Snook AE, Pires MM and Thevenin D: Ph-dependent grafting of cancer cells with antigenic epitopes promotes selective antibody-mediated cytotoxicity. J Med Chem 63(7): 3713-3722, 2020. PMID: 32196345. DOI: 10.1021/acs.jmedchem.0c00016

12 Schuerle S, Furubayashi M, Soleimany AP, Gwisai T, Huang W, Voigt C and Bhatia SN: Genetic encoding of targeted magnetic resonance imaging contrast agents for tumor imaging. ACS Synth Biol 9(2): 392-401, 2020. PMID: 31922737. DOI: 10.1021/acssynbio.9b00416
13 Marchiq I and Pouyssegur J: Hypoxia, cancer metabolism and the therapeutic benefit of targeting lactate/h(+) symporters. J Mol Med (Berl) 94(2): 155-171, 2016. PMID: 26099350. DOI: 10.1007/s00109-015-1307-x

14 Deiab S, Mazzio E, Messeha S, Mack N and Soliman KF: Highthroughput screening to identify plant derived human ldh-a inhibitors. European J Med Plants 3(4): 603-615, 2013. PMID: 24478981. DOI: $10.9734 /$ ejmp/2013/5995

15 Deiab S, Mazzio E, Eyunni S, McTier O, Mateeva N, Elshami F and Soliman KF: 1,2,3,4,6-penta-o-galloylglucose within galla chinensis inhibits human ldh-a and attenuates cell proliferation in mda-mb-231 breast cancer cells. Evid Based Complement Alternat Med 2015: 276946, 2015. PMID: 25918543. DOI: $10.1155 / 2015 / 276946$

16 Mack N, Mazzio EA, Bauer D, Flores-Rozas H and Soliman KF: Stable shrna silencing of lactate dehydrogenase a (ldha) in human mda-mb-231 breast cancer cells fails to alter lactic acid production, glycolytic activity, atp or survival. Anticancer Res 37(3): 1205-1212, 2017. PMID: 28314283. DOI: 10.21873/ anticanres. 11435

17 Zdralevic M, Brand A, Di Ianni L, Dettmer K, Reinders J, Singer K, Peter K, Schnell A, Bruss C, Decking SM, Koehl G, FelipeAbrio B, Durivault J, Bayer P, Evangelista M, O'Brien T, Oefner PJ, Renner K, Pouyssegur J and Kreutz M: Double genetic disruption of lactate dehydrogenases $\mathrm{a}$ and $\mathrm{b}$ is required to ablate the "warburg effect" restricting tumor growth to oxidative metabolism. J Biol Chem 293(41): 15947-15961, 2018. PMID: 30158244. DOI: $10.1074 / j b c . R A 118.004180$

18 de Padua MC, Delodi G, Vucetic M, Durivault J, Vial V, Bayer P, Noleto GR, Mazure NM, Zdralevic M and Pouyssegur J: Disrupting glucose-6-phosphate isomerase fully suppresses the "warburg effect" and activates oxphos with minimal impact on tumor growth except in hypoxia. Oncotarget 8(50): 87623-87637, 2017. PMID: 29152106. DOI: $10.18632 /$ oncotarget. 21007

19 Zdralevic M, Vucetic M, Daher B, Marchiq I, Parks SK and Pouyssegur J: Disrupting the 'warburg effect' re-routes cancer cells to oxphos offering a vulnerability point via 'ferroptosis'induced cell death. Adv Biol Regul 68: 55-63, 2018. PMID: 29306548. DOI: 10.1016/j.jbior.2017.12.002

20 Zdralevic M, Marchiq I, de Padua MMC, Parks SK and Pouyssegur J: Metabolic plasiticy in cancers-distinct role of glycolytic enzymes gpi, ldhs or membrane transporters mcts. Front Oncol 7: 313, 2017. PMID: 29326883. DOI: 10.3389/fonc. 2017.00313

21 Magnani E and Bettini E: Resazurin detection of energy metabolism changes in serum-starved pc12 cells and of neuroprotective agent effect. Brain Res Brain Res Protoc 5(3): 266-272, 2000. PMID: 10906492. DOI: $10.1016 / \mathrm{s} 1385-299 x(00) 00022-2$

22 Szklarczyk D, Gable AL, Lyon D, Junge A, Wyder S, HuertaCepas J, Simonovic M, Doncheva NT, Morris JH, Bork P, Jensen LJ and Mering CV: String v11: Protein-protein association networks with increased coverage, supporting functional discovery in genome-wide experimental datasets. Nucleic Acids Res 47(D1): D607-D613, 2019. PMID: 30476243. DOI: 10.1093/nar/gky1131

23 Szklarczyk D, Morris JH, Cook H, Kuhn M, Wyder S, Simonovic M, Santos A, Doncheva NT, Roth A, Bork P, Jensen LJ and von Mering C: The string database in 2017: Quality-controlled protein-protein association networks, made broadly accessible. Nucleic Acids Res 45(D1): D362-D368, 2017. PMID: 27924014. DOI: $10.1093 /$ nar/gkw937 
24 Grabacka M, Pierzchalska M, Dean M and Reiss K: Regulation of ketone body metabolism and the role of pparalpha. Int J Mol Sci 17(12), 2016. PMID: 27983603. DOI: 10.3390/ijms17122093

25 Liang H and Ward WF: Pgc-1alpha: A key regulator of energy metabolism. Adv Physiol Educ 30(4): 145-151, 2006. PMID: 17108241. DOI: 10.1152/advan.00052.2006

26 Cassim S, Raymond VA, Dehbidi-Assadzadeh L, Lapierre P and Bilodeau M: Metabolic reprogramming enables hepatocarcinoma cells to efficiently adapt and survive to a nutrient-restricted microenvironment. Cell Cycle 17(7): 903-916, 2018. PMID: 29633904. DOI: 10.1080/15384101.2018.1460023

27 Lacoste B, Raymond VA, Cassim S, Lapierre P and Bilodeau M: Highly tumorigenic hepatocellular carcinoma cell line with cancer stem cell-like properties. PLoS One 12(2): e0171215, 2017. PMID: 28152020. DOI: 10.1371/journal.pone.0171215

28 Song JP, Chen L, Chen X, Ren J, Zhang NN, Tirasawasdichai T, Hu ZL, Hua W, Hu YR, Tang HR, Chen HV and Hu SS: Elevated plasma beta-hydroxybutyrate predicts adverse outcomes and disease progression in patients with arrhythmogenic cardiomyopathy. Sci Transl Med 12(530): eaay8329, 2020. PMID: 32051229. DOI: 10.1126/scitranslmed.aay8329

29 Kim JT, Li C, Weiss HL, Zhou Y, Liu C, Wang Q and Evers BM: Regulation of ketogenic enzyme hmges2 by wnt/betacatenin/ppargamma pathway in intestinal cells. Cells 8(9), 2019. PMID: 31546785. DOI: 10.3390/cells8091106

30 Gharib E, Nasrinasrabadi P and Zali MR: Development and validation of a lipogenic genes panel for diagnosis and recurrence of colorectal cancer. PLoS One 15(3): e0229864, 2020. PMID: 32155177. DOI: 10.1371/journal.pone.0229864

31 Low END, Mokhtar NM, Wong Z and Raja Ali RA: Colonic mucosal transcriptomic changes in patients with long-duration ulcerative colitis revealed colitis-associated cancer pathways. J Crohns Colitis 13(6): 755-763, 2019. PMID: 30954025. DOI: 10.1093/ecco-jcc/jjz002

$32 \mathrm{Hu} \mathrm{D}$, Zhou M and Zhu X: Deciphering immune-associated genes to predict survival in clear cell renal cell cancer. Biomed Res Int 2019: 2506843, 2019. PMID: 31886185. DOI: 10.1155/2019/ 2506843

33 Xu WH, Xu Y, Wang J, Wan FN, Wang HK, Cao DL, Shi GH, Qu YY, Zhang HL and Ye DW: Prognostic value and immune infiltration of novel signatures in clear cell renal cell carcinoma microenvironment. Aging (Albany NY) 11(17): 6999-7020, 2019. PMID: 31493764. DOI: 10.18632/aging.102233

34 Li N, Li H, Cao L and Zhan X: Quantitative analysis of the mitochondrial proteome in human ovarian carcinomas. Endocr Relat Cancer 25(10): 909-931, 2018. PMID: 29997262. DOI: 10.1530/ERC-18-0243

35 Su SG, Yang M, Zhang MF, Peng QZ, Li MY, Liu LP and Bao SY: Mir-107-mediated decrease of hmgcs2 indicates poor outcomes and promotes cell migration in hepatocellular carcinoma. Int J Biochem Cell Biol 91(Pt A): 53-59, 2017. PMID: 28867541. DOI: 10.1016/j.biocel.2017.08.016

36 Chen SW, Chou CT, Chang CC, Li YJ, Chen ST, Lin IC, Kok SH, Cheng SJ, Lee JJ, Wu TS, Kuo ML and Lin BR: Hmges2 enhances invasion and metastasis via direct interaction with pparalpha to activate src signaling in colorectal cancer and oral cancer. Oncotarget 8(14): 22460-22476, 2017. PMID: 27816970. DOI: $10.18632 /$ oncotarget.13006

37 Saraon P, Cretu D, Musrap N, Karagiannis GS, Batruch I, Drabovich AP, van der Kwast T, Mizokami A, Morrissey C,
Jarvi K and Diamandis EP: Quantitative proteomics reveals that enzymes of the ketogenic pathway are associated with prostate cancer progression. Mol Cell Proteomics 12(6): 15891601, 2013. PMID: 23443136. DOI: 10.1074/mcp.M112. 023887

38 Kumamoto K, Nakachi Y, Mizuno Y, Yokoyama M, Ishibashi K, Kosugi C, Koda K, Kobayashi M, Tanakaya K, Matsunami T, Eguchi H, Okazaki Y and Ishida H: Expressions of 10 genes as candidate predictors of recurrence in stage III colon cancer patients receiving adjuvant oxaliplatin-based chemotherapy. Oncol Lett 18(2): 1388-1394, 2019. PMID: 31423202. DOI: 10.3892/ol.2019.10437

39 Martinez-Outschoorn UE, Lin Z, Whitaker-Menezes D, Howell A, Sotgia F and Lisanti MP: Ketone body utilization drives tumor growth and metastasis. Cell Cycle 11(21): 3964-3971, 2012. PMID: 23082722. DOI: 10.4161/cc.22137

$40 \mathrm{Hu} \mathrm{J}$ and $\mathrm{Yu} \mathrm{Y}$ : The function of thioredoxin-binding protein-2 (tbp-2) in different diseases. Oxid Med Cell Longev 2018. 4582130, 2018. PMID: 29854083. DOI: 10.1155/2018/4582130

41 Tang G, Duan F, Li W, Wang Y, Zeng C, Hu J, Li H, Zhang X, Chen $\mathrm{Y}$ and Tan H: Metformin inhibited nod-like receptor protein 3 inflammasomes activation and suppressed diabetes-accelerated atherosclerosis in apoe(-/-) mice. Biomed Pharmacother 119: 109410, 2019. PMID: 31518877. DOI: 10.1016/j.biopha.2019. 109410

42 Pouyssegur J, Franchi A, Salomon JC and Silvestre P: Isolation of a Chinese hamster fibroblast mutant defective in hexose transport and aerobic glycolysis: Its use to dissect the malignant phenotype. Proc Natl Acad Sci USA 77(5): 2698-2701, 1980. PMID: 6930659. DOI: 10.1073/pnas.77.5.2698

43 Stoltzman CA, Kaadige MR, Peterson CW and Ayer DE: Mondoa senses non-glucose sugars: Regulation of thioredoxin-interacting protein (txnip) and the hexose transport curb. J Biol Chem 286(44): 38027-38034, 2011. PMID: 21908621. DOI: 10.1074/jbc.M111.275503

44 Wu N, Zheng B, Shaywitz A, Dagon Y, Tower C, Bellinger G, Shen CH, Wen J, Asara J, McGraw TE, Kahn BB and Cantley LC: Ampk-dependent degradation of txnip upon energy stress leads to enhanced glucose uptake via glut1. Mol Cell 49(6): 11671175, 2013. PMID: 23453806. DOI: 10.1016/j.molcel.2013. 01.035

45 Jiang X, Pang Y, Zhao S, Hao H, Zhao X, Du W, Wang Y and Zhu H: Thioredoxin-interacting protein regulates glucose metabolism and improves the intracellular redox state in bovine oocytes during in vitro maturation. Am J Physiol Endocrinol Metab 318(3): E405-E416, 2020. PMID: 31935112. DOI: 10.1152/ajpendo.00057.2019

46 Qu X, Sun J, Zhang Y, Li J, Hu J, Li K, Gao L and Shen L: Cmyc-driven glycolysis via txnip suppression is dependent on glutaminase-mondoa axis in prostate cancer. Biochem Biophys Res Commun 504(2): 415-421, 2018. PMID: 30103944. DOI: 10.1016/j.bbrc.2018.08.069

47 Shen L, O'Shea JM, Kaadige MR, Cunha S, Wilde BR, Cohen $\mathrm{AL}$, Welm AL and Ayer DE: Metabolic reprogramming in triplenegative breast cancer through myc suppression of txnip. Proc Natl Acad Sci USA 112(17): 5425-5430, 2015. PMID: 25870263. DOI: $10.1073 /$ pnas.1501555112

48 Vassalli G: Aldehyde dehydrogenases: Not just markers, but functional regulators of stem cells. Stem Cells Int 2019: 3904645 , 2019. PMID: 30733805. DOI: 10.1155/2019/3904645 
49 Zhong G, Kirkwood J, Won KJ, Tjota N, Jeong H and Isoherranen $\mathrm{N}$ : Characterization of vitamin a metabolome in human livers with and without nonalcoholic fatty liver disease. J Pharmacol Exp Ther 370(1): 92-103, 2019. PMID: 31043436. DOI: 10.1124/jpet.119.258517

50 Wang Y, Wang CH, Zhang YF, Zhu L, Lei HM and Tang YB: Uplc-ms-based metabolomics reveals metabolic dysregulation in aldh1a1-overexpressed lung adenocarcinoma cells. Metabolomics 15(4): 52, 2019. PMID: 30911937. DOI: 10.1007/s11306-019$1514-5$

51 Calleja LF, Belmont-Diaz JA, Medina-Contreras O, Quezada H, Yoval-Sanchez B, Campos-Garcia J and Rodriguez-Zavala JS: Omeprazole as a potent activator of human cytosolic aldehyde dehydrogenase aldh1a1. Biochim Biophys Acta Gen Subj 1864(1): 129451, 2020. PMID: 31678145. DOI: 10.1016/j.bbagen.2019.129451

52 Fei $\mathrm{X}$, Wang $\mathrm{G}$, Shen $\mathrm{H}$ and Gu X: Placenta-specific 8 is a potential novel target for osimertinib resistance in non-small cell lung cancer. Oncol Lett 18(1): 955-961, 2019. PMID: 31289574. DOI: $10.3892 / \mathrm{ol} .2019 .10344$

53 McKinney A, Lindberg OR, Engler JR, Chen KY, Kumar A, Gong H, Lu KV, Simonds EF, Cloughesy TF, Liau LM, Prados M, Bollen AW, Berger MS, Shieh JTC, James CD, Nicolaides TP, Yong WH, Lai A, Hegi ME, Weiss WA and Phillips JJ: Mechanisms of resistance to egfr inhibition reveal metabolic vulnerabilities in human gbm. Mol Cancer Ther 18(9): 15651576, 2019. PMID: 31270152. DOI: 10.1158/1535-7163.MCT18-1330

54 Li L, Wang Y, Jiao L, Lin C, Lu C, Zhang K, Hu C, Ye J, Zhang $\mathrm{D}$, Wu $\mathrm{H}$, Feng $\mathrm{M}$ and $\mathrm{He} \mathrm{Y}$ : Protective autophagy decreases osimertinib cytotoxicity through regulation of stem cell-like properties in lung cancer. Cancer Lett 452: 191-202, 2019. PMID: 30910592. DOI: 10.1016/j.canlet.2019.03.027

55 Osmanov YI, Kogan EA, Rapoport LM, Teodorovich OV and Gaibov JA: [Markers of stem cells and their prognostic values for urothelial carcinomas of the urinary tract]. Urologiia (2): 40-49, 2019. PMID: 31162900.

56 Kim HM and Koo JS: Immunohistochemical analysis of cancer stem cell marker expression in papillary thyroid cancer. Front Endocrinol (Lausanne) 10: 523, 2019. PMID: 31428052. DOI: 10.3389/fendo.2019.00523

57 Elcheva IA, Wood T, Chiarolanzio K, Chim B, Wong M, Singh V, Gowda CP, Lu Q, Hafner M, Dovat S, Liu Z, Muljo SA and Spiegelman VS: Rna-binding protein igf2bp1 maintains leukemia stem cell properties by regulating hoxb4, myb, and aldh1a1. Leukemia, 2019. PMID: 31768017. DOI: 10.1038/s41375-0190656-9

58 Qin B, Zou S, Li K, Wang H, Wei W, Zhang B, Xiao L, Choi HH, Tang Q, Huang D, Liu Q, Pan Q, Meng M, Fang L and Lee MH: Csn6-trim21 axis instigates cancer stemness during tumorigenesis. Br J Cancer, 2020. PMID: 32225170. DOI: 10.1038/s41416-0200779-9

59 Tsunedomi R, Yoshimura K, Suzuki N, Hazama S and Nagano H: Clinical implications of cancer stem cells in digestive cancers: Acquisition of stemness and prognostic impact. Surg Today, 2020. PMID: 32025858. DOI: 10.1007/s00595-020-01968-x

60 Rebollido-Rios R, Venton G, Sanchez-Redondo S, Iglesias IFC, Fournet G, Gonzalez E, Romero Fernandez W, Borroto Escuela DO, Di Stefano B, Penarroche-Diaz R, Martin G, Ceylan I, Costello R and Perez-Alea M: Dual disruption of aldehyde dehydrogenases 1 and 3 promotes functional changes in the glutathione redox system and enhances chemosensitivity in nonsmall cell lung cancer. Oncogene 39(13): 2756-2771, 2020. PMID: 32015486. DOI: 10.1038/s41388-020-1184-9

61 Liu WT, Liu WB, Gao M, Zhang YY and Gu KS: Expression of aldh1a1 and cd133 is associated with the prognosis and effect of different chemotherapeutic regimens in gastric cancer. Oncol Lett 18(5): 4573-4582, 2019. PMID: 31611965. DOI: 10.3892/ol.2019. 10798

62 Liang D, Fan Y, Yang Z, Zhang Z, Liu M, Liu L and Jiang C: Discovery of coumarin-based selective aldehyde dehydrogenase 1a1 inhibitors with glucose metabolism improving activity. Eur J Med Chem 187: 111923, 2020. PMID: 31816557. DOI: 10.1016/j.ejmech.2019.111923

$63 \mathrm{Hu} \mathrm{Z,} \mathrm{Wu} \mathrm{J,} \mathrm{Qin} \mathrm{L,} \mathrm{Jin} \mathrm{H,} \mathrm{Lv} \mathrm{Y,} \mathrm{Zhang} \mathrm{R,} \mathrm{Xiao} \mathrm{C,} \mathrm{Cao} \mathrm{Y} \mathrm{and}$ Zhao Y: Aldh1a1 effect on yan yellow cattle preadipocyte differentiation. Anim Biotechnol: 1-10, 2019. PMID: 31646946. DOI: $10.1080 / 10495398.2019 .1679824$

64 Mori Y, Yamawaki K, Ishiguro T, Yoshihara K, Ueda H, Sato A, Ohata H, Yoshida Y, Minamino T, Okamoto K and Enomoto T: Aldh-dependent glycolytic activation mediates stemness and paclitaxel resistance in patient-derived spheroid models of uterine endometrial cancer. Stem Cell Reports 13(4): 730-746, 2019. PMID: 31564647. DOI: 10.1016/j.stemcr.2019.08.015

65 Namiki K, Wongsirisin P, Yokoyama S, Sato M, Rawangkan A, Sakai R, Iida K and Suganuma M: (-)-epigallocatechin gallate inhibits stemness and tumourigenicity stimulated by axl receptor tyrosine kinase in human lung cancer cells. Sci Rep 10(1): 2444, 2020. PMID: 32051483. DOI: 10.1038/s41598-020-59281-Z

66 Sun X, Song J, Li E, Geng H, Li Y, Yu D and Zhong C: ()epigallocatechin3gallate inhibits bladder cancer stem cells via suppression of sonic hedgehog pathway. Oncol Rep 42(1): 425435, 2019. PMID: 31180522. DOI: 10.3892/or.2019.7170

67 Sridharan S, Robeson M, Bastihalli-Tukaramrao D, Howard CM, Subramaniyan B, Tilley AMC, Tiwari AK and Raman D: Targeting of the eukaryotic translation initiation factor 4a against breast cancer stemness. Front Oncol 9: 1311, 2019. PMID: 31867270. DOI: 10.3389/fonc.2019.01311

68 Wang L, Wei Y, Ning C, Zhang M, Fan P, Lei D, Du J, Gale M, Ma Y and Yang Y: Ellagic acid promotes browning of white adipose tissues in high-fat diet-induced obesity in rats through suppressing white adipocyte maintaining genes. Endocr J 66(10): 923-936, 2019. PMID: 31292308. DOI: 10.1507/endocrj.EJ18-0467

69 Matsunaga T, Morikawa Y, Haga M, Endo S, Soda M, Yamamura K, El-Kabbani O, Tajima K, Ikari A and Hara A: Exposure to 9,10-phenanthrenequinone accelerates malignant progression of lung cancer cells through up-regulation of aldo-keto reductase 1b10. Toxicol Appl Pharmacol 278(2): 180-189, 2014. PMID: 24813866. DOI: 10.1016/j.taap.2014.04.024

70 Sato S, Genda T, Ichida T, Murata A, Tsuzura H, Narita Y, Kanemitsu Y, Ishikawa S, Kikuchi T, Mori M, Hirano K, Iijima K, Wada R, Nagahara A and Watanabe S: Impact of aldo-keto reductase family 1 member b10 on the risk of hepatitis c virusrelated hepatocellular carcinoma. J Gastroenterol Hepatol 31(7): 1315-1322, 2016. PMID: 26758591. DOI: 10.1111/jgh.13295

71 Mori M, Genda T, Ichida T, Murata A, Kamei M, Tsuzura H, Sato S, Narita Y, Kanemitsu Y, Ishikawa S, Kikuchi T, Shimada Y, Hirano K, Iijima K, Sugimoto K, Wada R, Nagahara A and Watanabe S: Aldo-keto reductase family 1 member b10 is associated with hepatitis b virus-related hepatocellular carcinoma 
risk. Hepatol Res 47(3): E85-E93, 2017. PMID: 27084455. DOI: 10.1111/hepr.12725

72 Qi LN, Li LQ, Chen YY, Chen ZH, Bai T, Xiang BD, Qin X, Xiao KY, Peng MH, Liu ZM, Liu TW, Qin X, Li S, Han ZG, Mo ZN, Santella RM, Winkler CA, O’Brien SJ and Peng T: Genomewide and differential proteomic analysis of hepatitis $\mathrm{b}$ virus and aflatoxin b1 related hepatocellular carcinoma in guangxi, china. PLoS One 8(12): e83465, 2013. PMID: 24391771. DOI: 10.1371/journal.pone.0083465

73 Feng G, Li XP, Niu CY, Liu ML, Yan QQ, Fan LP, Li Y, Zhang KL, Gao J, Qian MR, He N and Mi M: Bioinformatics analysis reveals novel core genes associated with nonalcoholic fatty liver disease and nonalcoholic steatohepatitis. Gene 742: 144549, 2020. PMID: 32184169. DOI: 10.1016/j.gene.2020.144549

74 Kanno M, Kawaguchi K, Honda M, Horii R, Takatori H, Shimakami T, Kitamura K, Arai K, Yamashita T, Sakai Y, Yamashita T, Mizukoshi E and Kaneko S: Serum aldo-keto reductase family 1 member b10 predicts advanced liver fibrosis and fatal complications of nonalcoholic steatohepatitis. J Gastroenterol 54(6): 549-557, 2019. PMID: 30707282. DOI: 10.1007/s00535-019-01551-3

75 Shi J, Chen L, Chen Y, Lu Y, Chen X and Yang Z: Aldo-keto reductase family 1 member b10 (akr1b10) overexpression in tumors predicts worse overall survival in hepatocellular carcinoma. J Cancer 10(20): 4892-4901, 2019. PMID: 31598161. DOI: $10.7150 /$ jca. 32768

76 Huang L, He R, Luo W, Zhu YS, Li J, Tan T, Zhang X, Hu Z and Luo D: Aldo-keto reductase family 1 member b10 inhibitors: Potential drugs for cancer treatment. Recent Pat Anticancer Drug Discov 11(2): 184-196, 2016. PMID: 26844556. DOI: 10.2174/ 1574892811888160304113346

77 Zhao SF, Wang SG, Zhao ZY and Li WL: Akr1c1-3, notably akr1c3, are distinct biomarkers for liver cancer diagnosis and prognosis: Database mining in malignancies. Oncol Lett $18(5)$ : 4515-4522, 2019. PMID: 31611960. DOI: 10.3892/ol.2019.10802

78 Sonohara F, Inokawa Y, Hishida M, Kanda M, Nishikawa Y, Yamada S, Fujii T, Sugimoto H, Kodera Y and Nomoto S: Prognostic significance of akr1b10 gene expression in hepatocellular carcinoma and surrounding non-tumorous liver tissue. Oncol Lett 12(6): 4821-4828, 2016. PMID: 28105190. DOI: $10.3892 / \mathrm{ol} .2016 .5240$

79 O'Reilly MW, House PJ and Tomlinson JW: Understanding androgen action in adipose tissue. J Steroid Biochem Mol Biol 143: 277-284, 2014. PMID: 24787657. DOI: 10.1016/ j.jsbmb.2014.04.008

80 Weber S, Salabei JK, Moller G, Kremmer E, Bhatnagar A, Adamski J and Barski OA: Aldo-keto reductase 1b15 (akr1b15): A mitochondrial human aldo-keto reductase with activity toward steroids and 3-keto-acyl-coa conjugates. J Biol Chem 290(10): 6531-6545, 2015. PMID: 25577493. DOI: 10.1074/jbc.M114. 610121

81 Rizner TL and Penning TM: Role of aldo-keto reductase family 1 (akr1) enzymes in human steroid metabolism. Steroids 79: 4963, 2014. PMID: 24189185. DOI: 10.1016/j.steroids.2013.10.012

82 Han C, Gao L, Bai H and Dou X: Identification of a role for serum aldo-keto reductase family 1 member b10 in early detection of hepatocellular carcinoma. Oncol Lett 16(6): 7123-7130, 2018. PMID: 30546447. DOI: 10.3892/ol.2018.9547

83 Hung JJ, Yeh YC and Hsu WH: Prognostic significance of akr1b10 in patients with resected lung adenocarcinoma. Thorac
Cancer 9(11): 1492-1499, 2018. PMID: 30253058. DOI: 10.1111/1759-7714.12863

84 Han C, Gao L, Zhao L, Sheng Q, Zhang C, An Z, Xia T, Ding Y, Wang J, Bai $\mathrm{H}$ and Dou X: Immunohistochemistry detects increased expression of aldo-keto reductase family 1 member b10 (akr1b10) in early-stage hepatocellular carcinoma. Med Sci Monit 24: 7414-7423, 2018. PMID: 30328412. DOI: 10.12659/ MSM.910738

85 DiStefano JK and Davis B: Diagnostic and prognostic potential of akr1b10 in human hepatocellular carcinoma. Cancers (Basel) 11(4), 2019. PMID: 30959792. DOI: 10.3390/cancers11040486

86 Seifert M, Peitzsch C, Gorodetska I, Borner C, Klink B and Dubrovska A: Network-based analysis of prostate cancer cell lines reveals novel marker gene candidates associated with radioresistance and patient relapse. PLoS Comput Biol 15(11): e1007460, 2019. PMID: 31682594. DOI: 10.1371/journal.pcbi. 1007460

87 Fang CY, Lin YH and Chen CL: Overexpression of akr1b10 predicts tumor recurrence and short survival in oral squamous cell carcinoma patients. J Oral Pathol Med 48(8): 712-719, 2019. PMID: 31237374 . DOI: 10.1111/jop.12891

88 Ko HH, Peng HH, Cheng SJ and Kuo MY: Increased salivary akr1b10 level: Association with progression and poor prognosis of oral squamous cell carcinoma. Head Neck 40(12): 2642-2647, 2018. PMID: 30430672. DOI: 10.1002/hed.25370

89 Ko HH, Cheng SL, Lee JJ, Chen HM, Kuo MY and Cheng SJ: Expression of akr1b10 as an independent marker for poor prognosis in human oral squamous cell carcinoma. Head Neck 39(7): 1327-1332, 2017. PMID: 28301069. DOI: 10.1002/hed. 24759

90 Cheng BY, Lau EY, Leung HW, Leung CO, Ho NP, Gurung S, Cheng LK, Lin CH, Lo RC, Ma S, Ng IO and Lee TK: Irak1 augments cancer stemness and drug resistance via the ap1/akr1b10 signaling cascade in hepatocellular carcinoma. Cancer Res 78(9): 2332-2342, 2018. PMID: 29483095. DOI: 10.1158/0008-5472.CAN-17-2445

91 Cubillos-Angulo JM, Fukutani ER, Cruz LAB, Arriaga MB, Lima JV, Andrade BB, Queiroz ATL and Fukutani KF: Systems biology analysis of publicly available transcriptomic data reveals a critical link between akr1b10 gene expression, smoking and occurrence of lung cancer. PLoS One 15(2): e0222552, 2020. PMID: 32097409. DOI: 10.1371/journal.pone.0222552

92 Chung YT, Matkowskyj KA, Li H, Bai H, Zhang W, Tsao MS, Liao J and Yang GY: Overexpression and oncogenic function of aldo-keto reductase family $1 \mathrm{~b} 10$ (akr1b10) in pancreatic carcinoma. Mod Pathol 25(5): 758-766, 2012. PMID: 22222635. DOI: 10.1038/modpathol.2011.191

93 Matkowskyj KA, Bai H, Liao J, Zhang W, Li H, Rao S, Omary $\mathrm{R}$ and Yang GY: Aldoketoreductase family $1 \mathrm{~b} 10$ (akr1b10) as a biomarker to distinguish hepatocellular carcinoma from benign liver lesions. Hum Pathol 45(4): 834-843, 2014. PMID: 24656094. DOI: $10.1016 /$ j.humpath.2013.12.002

94 Zemanova L, Hofman J, Novotna E, Musilek K, Lundova T, Havrankova J, Hostalkova A, Chlebek J, Cahlikova L and Wsol V: Flavones inhibit the activity of akr1b10, a promising therapeutic target for cancer treatment. J Nat Prod 78(11): 26662674, 2015. PMID: 26529431. DOI: 10.1021/acs.jnatprod.5b 00616

95 Jin YY, Han C, Geng N, Li YR, Zheng LY, Zhu WJ, Li YW, An ZY, Zhao LR, Wang JY, Dou XG and Bai H: [akr1b10 inhibitor 
enhances the inhibitory effect of sorafenib on liver cancer xenograft]. Zhonghua Gan Zang Bing Za Zhi 27(1): 39-44, 2019. PMID: 30685922. DOI: 10.3760/cma.j.issn.1007-3418.2019. 01.009

96 Seliger JM, Cicek SS, Witt LT, Martin HJ, Maser E and Hintzpeter J: Selective inhibition of human akr1b10 by $\mathrm{n}$ humulone, adhumulone and cohumulone isolated from humulus lupulus extract. Molecules 23(11), 2018. PMID: 30469331. DOI: 10.3390/molecules 23113041

97 Seliger JM, Misuri L, Maser E and Hintzpeter J: The hop-derived compounds xanthohumol, isoxanthohumol and 8-prenylnaringenin are tight-binding inhibitors of human aldo-keto reductases $1 \mathrm{~b} 1$ and 1b10. J Enzyme Inhib Med Chem 33(1): 607-614, 2018. PMID: 29532688. DOI: 10.1080/14756366.2018.1437728

98 Zeng CM, Chang LL, Ying MD, Cao J, He QJ, Zhu H and Yang B: Aldo-keto reductase akr1c1-akr1c4: Functions, regulation, and intervention for anti-cancer therapy. Front Pharmacol 8: 119, 2017. PMID: 28352233. DOI: 10.3389/fphar.2017.00119

99 Ischenko I, Seeliger H, Schaffer M, Jauch KW and Bruns CJ: Cancer stem cells: How can we target them? Curr Med Chem 15(30): 3171-3184, 2008. PMID: 19075661. DOI: 10.2174/09298 6708786848541

100 Cannon MJ, Ghosh D and Gujja S: Signaling circuits and regulation of immune suppression by ovarian tumor-associated macrophages. Vaccines (Basel) 3(2): 448-466, 2015. PMID: 26343197. DOI: $10.3390 /$ vaccines 3020448

101 Patel S, Barkell AM, Gupta D, Strong SL, Bruton S, Muskett FW, Addis PW, Renshaw PS, Slocombe PM, Doyle C, Clargo A, Taylor RJ, Prosser CE, Henry AJ, Robinson MK, Waters LC, Holdsworth G and Carr MD: Structural and functional analysis of dickkopf 4 (dkk4): New insights into dkk evolution and regulation of wnt signaling by dkk and kremen proteins. J Biol Chem 293(31): 12149-12166, 2018. PMID: 29925589. DOI: 10.1074/jbc.RA118.002918

102 Cruciat CM and Niehrs C: Secreted and transmembrane wnt inhibitors and activators. Cold Spring Harb Perspect Biol 5(3): a015081, 2013. PMID: 23085770. DOI: 10.1101/cshperspect. a015081

103 Eshelman MA, Shah M, Raup-Konsavage WM, Rennoll SA and Yochum GS: Tcf711 recruits ctbp and hdac1 to repress dickkopf4 gene expression in human colorectal cancer cells. Biochem Biophys Res Commun 487(3): 716-722, 2017. PMID: 28450117. DOI: 10.1016/j.bbrc.2017.04.123

104 Shimomura Y, Agalliu D, Vonica A, Luria V, Wajid M, Baumer A, Belli S, Petukhova L, Schinzel A, Brivanlou AH, Barres BA and Christiano AM: Apcdd1 is a novel wnt inhibitor mutated in hereditary hypotrichosis simplex. Nature 464(7291): 1043-1047, 2010. PMID: 20393562. DOI: 10.1038/nature08875

105 Mazzoni J, Smith JR, Shahriar S, Cutforth T, Ceja B and Agalliu D: The wnt inhibitor apcdd 1 coordinates vascular remodeling and barrier maturation of retinal blood vessels. Neuron 96(5): 10551069 e1056, 2017. PMID: 29154126. DOI: 10.1016/j.neuron. 2017.10.025

106 Cho SG: Apc downregulated 1 inhibits breast cancer cell invasion by inhibiting the canonical wnt signaling pathway. Oncol Lett 14(4): 4845-4852, 2017. PMID: 29085490. DOI: 10.3892/ol.2017. 6801

107 Ashktorab H, Daremipouran M, Devaney J, Varma S, Rahi H, Lee E, Shokrani B, Schwartz R, Nickerson ML and Brim H: Identification of novel mutations by exome sequencing in african american colorectal cancer patients. Cancer 121(1): 34-42, 2015. PMID: 25250560. DOI: $10.1002 / \mathrm{cncr} .28922$

108 Zhai W, Hu GH, Zheng JH, Peng B, Liu M, Huang JH, Wang GC, Yao XD and $\mathrm{Xu}$ YF: High expression of the secreted protein dickkopf homolog 4: Roles in invasion and metastasis of renal cell carcinoma and its association with von hippel-lindau gene. Int J Mol Med 33(5): 1319-1326, 2014. PMID: 24573574. DOI: $10.3892 /$ ijmm.2014.1673

109 Wang S, Wei H and Zhang S: Dickkopf-4 is frequently overexpressed in epithelial ovarian carcinoma and promotes tumor invasion. BMC Cancer 17(1): 455, 2017. PMID: 28666421. DOI: 10.1186/s12885-017-3407-1

110 Wang M, Ni B, Zhuang C, Zhao WY, Tu L, Ma XL, Yang LX, Zhang ZG and Cao H: Aberrant accumulation of dickkopf 4 promotes tumor progression via forming the immune suppressive microenvironment in gastrointestinal stromal tumor. Cancer Med 8(11): 5352-5366, 2019. PMID: 31353847. DOI: 10.1002/cam4.2437

111 Cai X, Yao Z, Li L and Huang J: Role of dkk4 in tumorigenesis and tumor progression. Int J Biol Sci 14(6): 616-621, 2018. PMID: 29904276. DOI: 10.7150/ijbs.24329

112 Pendas-Franco N, Garcia JM, Pena C, Valle N, Palmer HG, Heinaniemi M, Carlberg C, Jimenez B, Bonilla F, Munoz A and Gonzalez-Sancho JM: Dickkopf-4 is induced by tcf/beta-catenin and upregulated in human colon cancer, promotes tumour cell invasion and angiogenesis and is repressed by 1alpha,25dihydroxyvitamin $\mathrm{d} 3$. Oncogene 27(32): 4467-4477, 2008. PMID: 18408752. DOI: $10.1038 /$ onc. 2008.88

113 Ebert MP, Tanzer M, Balluff B, Burgermeister E, Kretzschmar AK, Hughes DJ, Tetzner R, Lofton-Day C, Rosenberg R, ReinacherSchick AC, Schulmann K, Tannapfel A, Hofheinz R, Rocken C, Keller G, Langer R, Specht K, Porschen R, Stohlmacher-Williams J, Schuster T, Strobel P and Schmid RM: Tfap2e-dkk4 and chemoresistance in colorectal cancer. N Engl J Med 366(1): 44-53, 2012. PMID: 22216841. DOI: 10.1056/NEJMoa1009473

$114 \mathrm{He} \mathrm{S}$, Shen J, Hu N, Xu X and Li J: Dkk4 enhances resistance to chemotherapeutics 5-fu and yn968d1 in colorectal cancer cells. Oncol Lett 13(2): 587-592, 2017. PMID: 28356933. DOI: $10.3892 / 01.2016 .5461$

115 Chen HJ, Sun J, Huang Z, Hou H, Jr., Arcilla M, Rakhilin N, Joe DJ, Choi J, Gadamsetty P, Milsom J, Nandakumar G, Longman R, Zhou XK, Edwards R, Chen J, Chen KY, Bu P, Wang L, Xu Y, Munroe R, Abratte C, Miller AD, Gumus ZH, Shuler M, Nishimura N, Edelmann $\mathrm{W}$, Shen $\mathrm{X}$ and Lipkin SM: Comprehensive models of human primary and metastatic colorectal tumors in immunodeficient and immunocompetent mice by chemokine targeting. Nat Biotechnol 33(6): 656-660, 2015. PMID: 26006007. DOI: 10.1038/nbt.3239

116 Zeng S, Seifert AM, Zhang JQ, Cavnar MJ, Kim TS, Balachandran VP, Santamaria-Barria JA, Cohen NA, Beckman MJ, Medina BD, Rossi F, Crawley MH, Loo JK, Maltbaek JH, Besmer P, Antonescu CR and DeMatteo RP: Wnt/beta-catenin signaling contributes to tumor malignancy and is targetable in gastrointestinal stromal tumor. Mol Cancer Ther 16(9): 1954-1966, 2017. PMID: 28611108. DOI: $10.1158 / 1535-7163$.MCT-17-0139

117 Chouhan S, Singh S, Athavale D, Ramteke P, Pandey V, Joseph J, Mohan R, Shetty PK and Bhat MK: Glucose induced activation of canonical wnt signaling pathway in hepatocellular carcinoma is regulated by dkk4. Sci Rep 6: 27558, 2016. PMID: 27272409. DOI: $10.1038 /$ srep27558 
118 Hirata H, Hinoda Y, Majid S, Chen Y, Zaman MS, Ueno K, Nakajima K, Tabatabai ZL, Ishii N and Dahiya R: Dickkopf-4 activates the noncanonical c-jun-nh2 kinase signaling pathway while inhibiting the wnt-canonical pathway in human renal cell carcinoma. Cancer 117(8): 1649-1660, 2011. PMID: 21472712. DOI: $10.1002 /$ cncr.25666

119 Chau WK, Ip CK, Mak AS, Lai HC and Wong AS: C-kit mediates chemoresistance and tumor-initiating capacity of ovarian cancer cells through activation of wnt/beta-catenin-atp-binding cassette g2 signaling. Oncogene 32(22): 2767-2781, 2013. PMID: 22797058. DOI: $10.1038 /$ onc. 2012.290

120 Dakhova O, Rowley D and Ittmann M: Genes upregulated in prostate cancer reactive stroma promote prostate cancer progression in vivo. Clin Cancer Res 20(1): 100-109, 2014. PMID: 24150235. DOI: 10.1158/1078-0432.CCR-13-1184

121 Humbert M, Casteran N, Letard S, Hanssens K, Iovanna J, Finetti P, Bertucci F, Bader T, Mansfield CD, Moussy A, Hermine O and Dubreuil P: Masitinib combined with standard gemcitabine chemotherapy: In vitro and in vivo studies in human pancreatic tumour cell lines and ectopic mouse model. PLoS One 5(3): e9430, 2010. PMID: 20209107. DOI: 10.1371/journal.pone. 0009430

122 Gu X, Ma Y, Xiao J, Zheng H, Song C, Gong Y and Xing X: Upregulated biglycan expression correlates with the malignancy in human colorectal cancers. Clin Exp Med 12(3): 195-199, 2012. PMID: 21879307. DOI: 10.1007/s10238-011-0155-4

123 Guo D, Zhang W, Yang H, Bi J, Xie Y, Cheng B, Wang Y and Chen $\mathrm{S}$ : Celastrol induces necroptosis and ameliorates inflammation via targeting biglycan in human gastric carcinoma. Int J Mol Sci 20(22), 2019. PMID: 31739592. DOI: 10.3390/ijms20225716

124 Hu L, Duan YT, Li JF, Su LP, Yan M, Zhu ZG, Liu BY and Yang QM: Biglycan enhances gastric cancer invasion by activating fak signaling pathway. Oncotarget 5(7): 1885-1896, 2014. PMID: 24681892. DOI: $10.18632 /$ oncotarget. 1871

125 Xing X, Gu X and Ma T: Knockdown of biglycan expression by RNA interference inhibits the proliferation and invasion of, and induces apoptosis in, the hct116 colon cancer cell line. Mol Med Rep 12(5): 7538-7544, 2015. PMID: 26459740. DOI: $10.3892 / \mathrm{mmr} .2015 .4383$
126 Endo Y, Ishiwata-Endo H and Yamada KM: Cell adhesion to anosmin via alpha5beta1, alpha4beta1, and alpha9beta1 integrins. Cell Adh Migr 12(2): 93-100, 2018. PMID: 27715389. DOI: $10.1080 / 19336918.2016 .1221568$

127 Theocharis AD and Karamanos NK: Proteoglycans remodeling in cancer: Underlying molecular mechanisms. Matrix Biol 75-76. 220-259, 2019. PMID: 29128506. DOI: 10.1016/j.matbio.2017. 10.008

128 Dos Reis DC, Damasceno KA, de Campos CB, Veloso ES, Pegas GRA, Kraemer LR, Rodrigues MA, Mattos MS, Gomes DA, Campos PP, Ferreira E, Russo RC and Cassali GD: Versican and tumor-associated macrophages promotes tumor progression and metastasis in canine and murine models of breast carcinoma. Front Oncol 9: 577, 2019. PMID: 31334111. DOI: 10.3389/fonc. 2019.00577

129 Hart ML, Rusch E, Kaupp M, Nieselt K and Aicher WK: Expression of desmoglein 2, desmocollin 3 and plakophilin 2 in placenta and bone marrow-derived mesenchymal stromal cells. Stem Cell Rev Rep 13(2): 258-266, 2017. PMID: 28154962. DOI: 10.1007/s12015-016-9710-4

130 Rotzer V, Hartlieb E, Winkler J, Walter E, Schlipp A, Sardy M, Spindler V and Waschke J: Desmoglein 3-dependent signaling regulates keratinocyte migration and wound healing. J Invest Dermatol 136(1): 301-310, 2016. PMID: 26763450. DOI: 10.1038/JID.2015.380

131 Kamioka H, Tomono T, Fujita A, Onozato R, Iijima M, Tsuchida S, Arai T, Fujita Y, Zhang X, Yano K and Ogihara T: Moesinmediated p-glycoprotein activation during snail-induced epithelial-mesenchymal transition in lung cancer cells. J Pharm Sci, 2020. PMID: 32173323. DOI: 10.1016/j.xphs.2020.03.008

$132 \mathrm{Yu} \mathrm{L}$, Zhao L, Wu H, Zhao H, Yu Z, He M, Jin F and Wei M: Moesin is an independent prognostic marker for er-positive breast cancer. Oncol Lett 17(2): 1921-1933, 2019. PMID: 30675256. DOI: $10.3892 / \mathrm{ol} .2018 .9799$

Received May 26, 2020

Revised June 14, 2020

Accepted June 26, 2020 